\title{
Current state and perspectives of Space Weather science in Italy
}

\author{
Christina Plainaki ${ }^{1}$,*, Marco Antonucci ${ }^{2}$, Alessandro Bemporad ${ }^{3}$, Francesco Berrilli ${ }^{4}$, Bruna Bertucci ${ }^{5}$, \\ Marco Castronuovo ${ }^{1}$, Paola De Michelis ${ }^{6}$, Marco Giardino ${ }^{1}$, Roberto Iuppa ${ }^{7,10}$, Monica Laurenza ${ }^{8}$, \\ Federica Marcucci ${ }^{8}$, Mauro Messerotti ${ }^{9}$, Livio Narici ${ }^{4}$, Barbara Negri ${ }^{1}$, Francesco Nozzoli ${ }^{10}$, \\ Stefano Orsini ${ }^{8}$, Vincenzo Romano ${ }^{6}$, Enrico Cavallini ${ }^{1}$, Gianluca Polenta ${ }^{1}$, and Alessandro Ippolito ${ }^{1}$ \\ ${ }^{1}$ ASI - Agenzia Spaziale Italiana, Via del Politecnico snc, 00133 Rome, Italy \\ 2 Aeronautica Militare Italiana, Stato Maggiore Aeronautica $-3^{\circ}$ Reparto, Viale dell'Università n.4, 00185 Rome, Italy \\ ${ }^{3}$ INAF-Osservatorio Astrofisico di Torino, via Osservatorio 20, 10025 Pino Torinese, Torino, Italy \\ ${ }^{4}$ Università di Roma Tor Vergata, Dipartimento di Fisica, Via Ricerca Scientifica 1, 00133 Rome, Italy \\ ${ }^{5}$ Università di Perugia, Dipartimento di Fisica e Geologia, Via Pascoli s.n.c., 06124 Perugia, Italy \\ 6 Istituto Nazionale di Geofisica e Vulcanologia, Via di Vigna Murata 605, 00143 Rome, Italy \\ ${ }^{7}$ Università di Trento, Dipartimento di Fisica, via Sommarive 14, 38123 Trento, Italy \\ ${ }^{8}$ INAF-Istituto di Astrofisica e Planetologia Spaziali, Via del Fosso del Cavaliere 100, 00133 Rome, Italy \\ 9 INAF- Osservatorio Astronomico di Trieste, Loc. Basovizza n. 302, 34149 Trieste, Italy \\ ${ }^{10}$ INFN-TIFPA, via Sommarive 14, 38123 Trento, Italy
}

Received 25 March 2019 / Accepted 27 December 2019

\begin{abstract}
Italian teams have been involved many times in Space Weather observational campaigns from space and from the ground, contributing in the advancing of our knowledge on the properties and evolution of the related phenomena. Numerous Space Weather forecasting and now-casting modeling efforts have resulted in a remarkable add-on to the overall progress in the field, at both national and international level. The Italian Space Agency has participated several times in space missions with science objectives related to Space Weather; indeed, an important field for the Italian scientific and industrial communities interested in Heliophysics and Space Weather, is the development of new instrumentation for future space missions. In this paper, we present a brief state-of-the-art in Space Weather science in Italy and we discuss some ideas on a long-term plan for the support of future scientific research in the related disciplines. In the context of the current roadmap, the Italian Space Agency aims to assess the possibility to develop a national scientific Space Weather data centre to encourage synergies between different science teams with interest in the field and to motivate innovation and new mission concept development. Alongside with the proposed recommendations, we also discuss how the Italian expertise could complement international efforts in a wider international Space Weather context.
\end{abstract}

Keywords: heliosphere / instrumentation / missions / strategy / data management

\section{Introduction: science case and scope of this roadmap}

Circumterrestrial Space Weather (often referred to, simply, as "Space Weather") has its main origin at the Sun being driven by the solar activity (e.g., flares, Coronal Mass Ejections CMEs) and characterized by its effects in the Earth's magnetosphere and upper atmosphere. High energy particles of non-solar origin, such as the Galactic Cosmic Rays (GCRs),

\footnotetext{
*Corresponding author: christina.plainaki@asi.it
}

can also influence Space Weather in the Solar System through their interplay with the Heliosphere. Space Weather is manifested through a series of phenomena including Solar Energetic Particle (SEP), geomagnetic variability, and Ground Level Enhancement (GLE) events, as well as variations of the GCR intensity. Charged particle precipitation in the Earth's polar atmosphere causes auroras, and increases the conductivity of the lower ionosphere in the auroral electrojet. Moreover, the energy input in the polar region heats the thermosphere, changing the distribution of the atmospheric constituents and hence the ionospheric electron density profile (Denton et al., 2009). Also the Earth's troposphere has a role in the Space Weather 


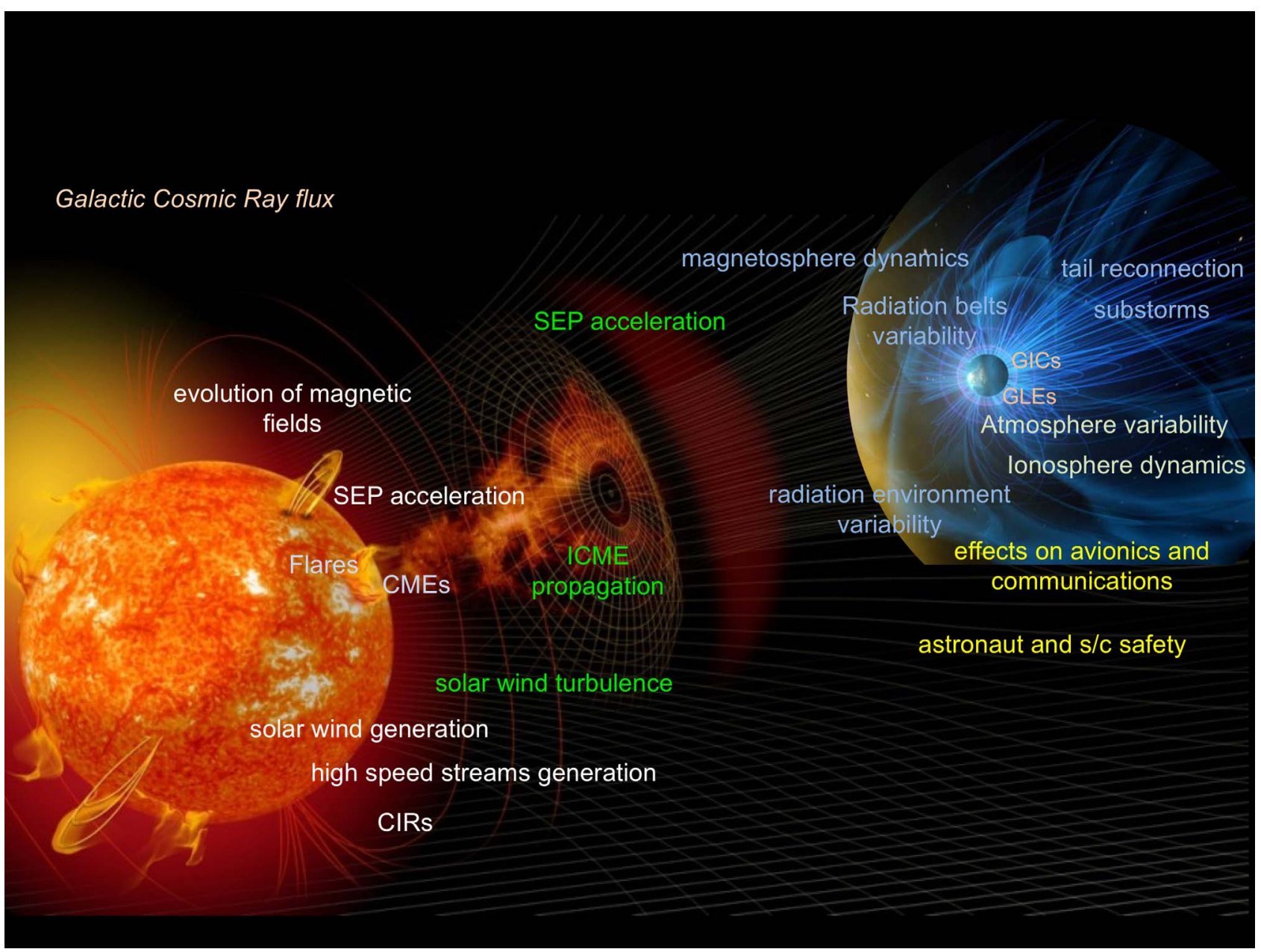

Fig. 1. Space Weather phenomena resulting from the Sun-Earth connection and/or its interplay with the galactic cosmic radiation. Background figures are from NASA.

chain since the convective systems within can generate gravity waves that transport energy and momentum into the upper atmosphere; the dissipation of such waves is a significant source of heat in the thermosphere (alongside the solar EUV radiation). A graphical overview of the dominant Space Weather phenomena and coupling mechanisms resulting from the Sun-Earth connection is presented in Figure 1.

Space Weather can have impacts on space-based and terrestrial technological infrastructures and biological systems. Sporadic events caused by powerful eruptions on the Sun can disrupt high frequency communications, degrade the precision of the navigation systems, provoke perturbations in the long conductive transmission lines, gas pipelines, railway systems, influence satellite functions and (in extreme cases) cause the loss of the mission. Moreover, Space Weather events can be hazardous for the health of aircrew members and flight passengers (especially on polar flights), and astronauts. It is therefore important to mitigate the risks of Space Weather impacts on technology, infrastructure, navigation, health and human activities, based on:

- our knowledge of the physics of the Sun, the interplanetary space, the Earth's environment (magnetosphere, ionosphere, atmosphere) and its interior, and their coupling at long and short timescales;

- the use of scientific research products for the development of reliable systems for Space Weather forecasting originating, preferably, from multi-data observations.

The Space Weather discipline has both scientific and operational aspects which are unavoidably strongly related to one another. Koskinen et al. (2017) characterized Space Weather as "science with applications" pointing out that the progress needed to improve both short- and long-term forecasting challenges our understanding of the scientific foundations. It is now evident that to address the requirements of future Space Weather services we need to advance our insights in and understanding of Space Weather science. Indeed, there is an ongoing growth of interest in Space Weather research (see Fig. 2) that goes beyond individual scientific disciplines or national capabilities: Space Weather is a global challenge requiring observational resources that cover the whole world providing, when possible, detailed information on physical parameters characterizing the interplanetary space and its interplay with the Earth's environment.

In a 2009 definition agreed among 24 countries (Lilensten \& Belehaki 2009), it was defined that "Space Weather is the 


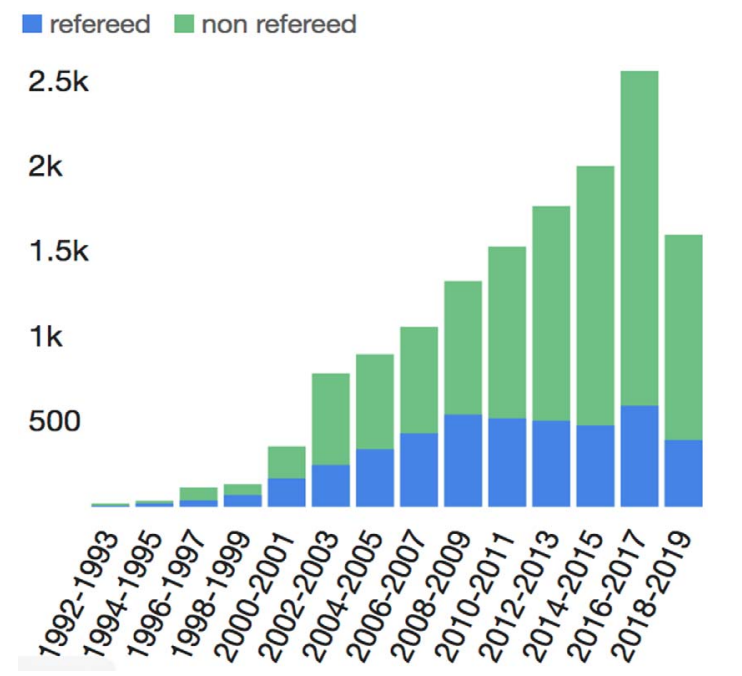

Fig. 2. Number of publications per year with the term "Space Weather" included in the abstract in NASA/Astrophysics Data System (refereed in blue; non refereed in green).

physical and phenomenological state of natural space environments. The associated discipline aims, through observation, monitoring, analysis and modeling, at understanding and predicting the state of the Sun, the interplanetary and planetary environments, and the solar and non-solar driven perturbations that affect them; and also at forecasting and now-casting the possible impacts on biological and technological systems". Based on this definition, and from a wider perspective, Space Weather refers to the entire Solar System. The Space Weather conditions around the Earth or, in general, around a planetary body within our Solar System, are strongly determined by the interactions between the body in question and its local space environment. The study of either circumterrestrial or planetary Space Weather considers different cross-disciplinary topics, including the physics of the Sun, the interaction of the solar wind and/or of magnetospheric plasmas with planetary/satellite surfaces and thick or tenuous atmospheres and ionospheres; the variability of the planetary magnetospheric regions under different external plasma conditions; the interactions of planetary radiation belts with atmospheres, satellites and rings (Lilensten et al., 2014; Plainaki et al., 2016). The lessons learned from the study of the interactions of planetary bodies with plasma, energetic particles and solar photon radiation can be an important feedback for an in depth understanding of the circumterrestrial Space Weather phenomena. Moreover, the advanced understanding of planetary Space Weather conditions is a key point for the robotic Solar System exploration.

Another area where Space Weather plays an important role is the radiation risk assessment in the context human space exploration. The understanding of the potential risk for the crew due to the increased level of radiation during a SEP event is mandatory for issuing proper upgrades to mission plans and providing optimized countermeasures for risk mitigation. Two major fields of scientific research and technology involved in this endeavor are radiobiology and radiation monitoring. In parallel, systematic research addressing how SEP events are generated - both the short-lived (abrupt) events associated with flares and the long-lived (gradual) events associated with CME shocks - is necessary. This is an important application of fundamental plasma physics to cosmic plasmas (involving, for instance, the investigation of particle energization in magnetic reconnection diffusion regions and in shocks). Moreover, research should also focus on the detailed understanding of how SEPs propagate through the interplanetary space (considering also the possible perturbation of the particle propagation paths by CMEs and Co-rotating Interaction Regions - CIRs) and get scattered by magnetic irregularities. Testing, development, and validation of now-casting SEP event strategies and models require knowledge obtained through research in the aforementioned areas. In addition to the radiobiological issue and from a broader Space Weather perspective, the concept should also include the risk of electronic malfunctioning due to radiation, as the crew life is strongly linked to the proper functioning of all the electronic tools and critical control systems in the space vessel. The understanding of the radiationinduced interference with spacecraft systems, therefore, is of fundamental importance.

Space Weather effects on spacecraft functions are mainly due to solar particle effects on electronics and materials. Solar particle events can cause solar cell degradation, star-tracker problems, memory device problems, and noise on imaging systems (Keil, 2007). In addition, the presence of a geomagnetic storm may require spacecraft operations actions to correct orientation. The direct consequences of space radiation induced by Space Weather include, therefore, Single Event Effects (SEE) on powered electronics (Single Event Upsets - SEU; Single Event Latch-up - SEL; Single Event Gate Rupture - SEGR; Single Event Burnout - SEB), Total Ionizing Dose (TID), and displacement damages. In summary, Space Weather can cause problems in both payload and platform system functionality. In particular, the sensitivity and noise level, the scientific instruments, sensors (e.g., the Charged-Coupled Devices - CCDs), and windows can be seriously influenced. Power subsystems, avionics, propulsion, on board data handling, telemetry and telecommunications, as well as the thermal subsystem can be also affected (Keil, 2007).

In aviation, in addition to risks due to SEE on electronics, Space Weather may provoke the degradation of radio/satellite communications (e.g., High Frequency (HF)) and satellite communication disturbances), on board system failure due to radiation, high radiation doses received by airplane passengers and crew, problems in the Global Navigation Satellite System (GNSS) radio signal transmittance resulting in position and timing errors, and degradation of the accuracy of magnetic based equipment and compasses.

The constant monitoring of the changes in the Earth's magnetic field permits, among others, the registration of sudden and intense geomagnetic field variations. Such events may result in the generation (in the electrically conducting surface of the Earth) of geoelectric fields which give rise to Geomagnetically Induced Currents (GICs). GICs driven by Space Weather have the potential to damage critical infrastructures (e.g., power grids) causing, often, their malfunction. Possible related impacts on local infrastructures need, therefore, deeper assessment; in this context, the geomagnetic data play a fundamental role (Tozzi et al., 2019a, 2019b). The magnetic field measurements on the ground and in space can also be used to investigate the 
role that turbulent processes play in the framework of solar wind-magnetosphere-ionosphere coupling and in the generation of plasma inhomogeneities and irregularities in the ionosphere, responsible for the delay, distortion or even total loss of electromagnetic signals while passing through the ionosphere (De Michelis \& Consolini, 2015; De Michelis et al., 2015, 2016, 2017). This means that turbulence is also able to seriously compromise the performance of the Global Positioning System (GPS) and the GNSS. Therefore, an in depth investigation of the role of turbulent processes in solar wind-magnetosphereionosphere coupling is a necessary requisite for understanding Space Weather.

The Space Weather Italian Community (SWICo) has been involved many times in observational campaigns from space and from the ground, often with lead roles, providing important insights in Space Weather science. Moreover, the Italian scientific community is very active in the development of new instrumentation for future space missions and groundbased observations. The scientific need to construct new space-based and ground-based instrumentation for Space Weather observations has been already identified, at international level, as of high priority (see for instance, the COSPAR and ILWS Space Weather roadmap; Schrijver et al. 2015). The need for a high resolution imaging of the solar corona and solar vector observations (e.g., to quantify activity from active regions), an expansion of the in situ coverage of particle acceleration regions (e.g., to determine the space environment in the vicinity of current or future spacecraft), the dense spatial coverage of particle and field instruments (from Low Earth Orbit-LEO to Geostationary Orbit-GEO), and the measurement of the long-term variability of the space environment, are some of the main drivers for the deployment of new or additional instrumentation for Space Weather purposes. Finally, as we discuss in Section 2, different forecasting and now-casting modeling efforts have contributed to the overall progress in Space Weather, at both national and international level.

Scope of this roadmap: This roadmap, agreed among experts from different Italian Institutions, provides a general perspective for the development of scientific Space Weather activities in Italy. It identifies the main areas where further work is needed and it provides recommendations to achieve this objective, taking into consideration the existent observational and modeling capabilities that support Space Weather research in Italy. The current roadmap can be therefore considered as a proposal for a long-term strategy, which, however, may well be modified and/or integrated in the next years on the basis of possible new top level science needs.

In the context of this strategy, the possibility to develop a national scientific Space Weather data center, to encourage synergies between different science teams and to allow the most efficient access to multi-disciplinary data, will be assessed. Such a scientific data center could be allocated in the Space Science Data Center (SSDC) of the Italian Space Agency (ASI) and it could host both Space Weather data archives and related tools. Optimization of the data observational coverage, homogenization - as far as possible - of the data based on internationally recognized standards, and harmonization of the access to data archives would be the initial goals of the related project. The so called ASI Space weather InfraStructure (ASPIS) will function as a multi-dimensional tool for science, nevertheless, its design and overall configuration should take into account the need of the international community for future Space Weather services. Future implementation of such services by different institutions could benefit significantly by the interdisciplinary nature of the ASPIS data.

This paper is organized as follows. In Section 2, we briefly present the state-of-the-art in Space Weather research in Italy highlighting the novelty of the related scientific results and outputs. In Section 3, we discuss the key challenges in Space Weather research from an international point of view. In Section 4, we provide the roadmap's detailed recommendations classified in six categories: Observational and theoretical research recommendations (Sect. 4.1); Maintenance of existing facilities (Sect. 4.2); Study of space mission concepts and deployment of new instrumentation (Sect. 4.3); Development of a national scientific Space Weather data centre (Sect. 4.4); Teaming and collaboration between ASI and the scientific community (Sect. 4.5); Education, training, and public outreach (Sect. 4.6). The conclusions of the current analysis are presented in Section 5, where some future perspectives in the context of a more global approach are also discussed.

\section{State-of-the-art in Space Weather science in Italy}

The Italian scientific community has a long experience in theoretical, modeling, and observation-based research covering a wide range of thematic areas related to Space Weather. In particular, the Space Weather scientific research in Italy has been mainly focused on the following fields:

(I) Solar physics, including the study of the Sun from its photosphere to the corona with emphasis on the study of the structure and evolution of magnetic regions on the Sun, solar flares, coronal magnetic structures, solar wind and energetic particle generation processes, solar radio emission mechanisms.

(II) Solar-terrestrial physics, including the study of the solar wind, the propagation and evolution of CMEs, High Speed Streams (HSSs) and SEPs from the Sun to geospace, the solar wind-magnetosphere coupling, the interactions of SEPs with the Earth's magnetosphere and atmosphere, and the generation of GLE events.

(III) Geomagnetism, including the study of the geomagnetic disturbances (e.g., pulsations, storms and substorms) and GICs.

(IV) Ionosphere and upper atmosphere physics, including the study of the magnetosphere-ionosphere coupling, of the thermosphere response to solar activity, of the aurora and of ionospheric storms.

(V) Planetary Space Weather science, with emphasis on the study of interactions between the solar wind and planetary magnetospheres/atmospheres, including the investigations of auroras, and the interplay between charged particle populations and lunar environments within the systems of giant planets.

(VI) Galactic cosmic ray physics, with particular emphasis on the study of the GCR modulation and its connection to the solar activity. 
Table 1. Classification of Space Weather related projects in which the Italian scientific community has participated based on topic areas. The numbering of the scientific research fields has been defined as follows: I: Solar physics; II: Solar-terrestrial physics; III: Geomagnetism; IV: Ionosphere and upper atmosphere physics; V: Planetary Space Weather science; VI: Galactic cosmic ray physics; VII: Technological and biological impacts study. Technical details considering each project can be found in the respective topic annex within Appendix A.

\begin{tabular}{|c|c|c|c|}
\hline Topic area & $\begin{array}{l}\text { Scientific research } \\
\text { field }\end{array}$ & Projects & $\begin{array}{l}\text { Annex within } \\
\text { Appendix A }\end{array}$ \\
\hline Flares, active regions, coronal magnetic structures & I & F-CHROMA; HESPE; FLARECAST & Topic Annex n. 1 \\
\hline Ionosphere and plasmasphere & IV, II, III & ESPAS; PLASMON & Topic Annex n. 3 \\
\hline Particle radiation & VI, VII, V, I, II & e-HEROES; CORA & Topic Annex n. 4 \\
\hline Infrastructure and service development & I, II, III, IV, VI, VII & $\begin{array}{l}\text { HELIO; NMDB; SWERTO; SOLID; SWIFF; } \\
\text { SOLARNET; GREST; PRE-EST; IPS; PECASUS; } \\
\text { "Space Weather Service" by AMI }\end{array}$ & Topic Annex n. 5 \\
\hline
\end{tabular}

(VII) Technological and biological impacts study, including Radio Frequency Interferences (RFIs) in satellite communications, radiation risk assessment in the context human space exploration, and analysis of electronics and system malfunctioning onboard space vessels due to Space Weather.

The contribution of the Italian scientific community to national and international research activities covering the aforementioned fields is manifested through its wide participation in projects of rapidly increasing interest, often with lead or co-lead roles.

\subsection{Major recent and on-going projects related to Space Weather}

Current projects in the field of Space Weather are in general focused on the scientific aspects of the related disciplines, or possible technological applications and services, or both. In this section, the main projects in the field of Space Weather, either recently concluded or ongoing, with Italian participation or leadership, including rigorously scientific efforts or operational activities or both, are presented and discussed. We anticipate that the list of the projects provided here is not exhaustive, nevertheless, it represents well the thematic areas covered by the Italian Space Weather community and the methodological approaches followed through the years. We provide below an overview of these projects and capabilities, organized within topic areas belonging to the scientific research fields listed in the beginning of Section 2 (see Table 1). This overview shows how these topics highlight major themes in Italian Space Weather research, indicating, where possible, linkages and synergies between projects. Technicalities and managerial aspects (where relevant) related to these projects, organized into topic annexes, are listed in Appendix A. To facilitate reading, we provide a linkage between the topic areas of the projects discussed in the paper and the related annexes within Appendix A (see Table 1).

Topic area n. 1: Flares, active regions, coronal magnetic structures

To understand the physics behind Space Weather, it is important to speculate on Space Weather sources, from both an observational and modeling point of view. An important step toward this direction was made through the FP7 "Flare
Chromospheres: Observations, models and archives" (FCHROMA) project (2013-2017), which was devoted to the analysis and interpretation of the ground- and space-based observational data of solar flares, to their testing against mode-predictions and to the development of an archive of both solar flare observations and models. Among the key science challenges of this project was the identification of the energy output of the flaring chromosphere, a major theme in Space Weather research. Through the linking of the observations to the available models, this project provided some important feedback to be potentially linked to other efforts in the field as well; in particular, the first simultaneous observations of a flare in $\mathrm{H} \alpha$ and $\mathrm{H} \beta$ were made (Capparelli et al., 2017), and the viability of an Alfvén wave energy transport model as an alternative to the long-standing electron beam model for producing chromospheric flare radiation was demonstrated (Kerr et al., 2016). Moreover, a flare where the absence of hydrogen Balmer lines may rule out any significant role for electrons in impulsive phase energy transport was identified and studied (Procházka et al., 2017).

Solar flares have been also studied extensively through hard $\mathrm{X}$-ray measurements. Hard X-ray data provide a direct observational link to the acceleration and transport of highly energetic particles in solar flares, a key aspect of Space Weather. Exploitation of high energy solar physics data in Europe was possible through the FP7 "High Energy Solar Physics Data in Europe" (HESPE) project (2010-2013), which included theoretical, computational and technological activities. In particular, the computational activity was focused on the application of mathematical techniques to efficiently extract information out of the data (e.g., Massone \& Piana, 2013). One of the major key aspects highlighted by the HESPE project was the rapid calculation of the detailed physics of the processes leading to the observed X-ray radiation (e.g., Guo et al., 2012a, 2012b). Such techniques can be particularly useful when analyzing big data hence opening a synergetic pathway toward Space Weather data-exploitation at large.

Statistical and machine learning techniques have been recently applied in the context of the H2020 Flare Likelihood And Region Eruption forecasting (FLARECAST) project (2015-2017). Within FLARECAST, for the first time, a sophisticated and automated forecasting system for solar flares was developed. The primary science objectives of FLARECAST were to understand the drivers of flare activity and to improve flare prediction. At the same time, this project provided a 
globally accessible flare prediction service and engaged with Space Weather end users.

For further details on the projects in this topic area, the reader is referred to Appendix A (Topic Annex n. 1).

\section{Topic area n. 2: Space plasmas}

Understanding the collective and dissipative processes responsible for the energy transfer from larger to smaller scales, is a major theme in space physics and Space Weather science. The way turbulence develops and energy is partitioned between larger and smaller scales were among the challenge questions that the FP7 "Solar system plasma Turbulence: Observations, inteRmittency and Multifractals" (STORM) project (20132015) tried to address. Analysis of solar wind data by Ulyses and plasma and magnetic field data from Cluster, Venus Express, Giotto, and Cassini satellites provided new insights into plasma and field fluctuations and offered the chance to probe the smallest scales ever explored in the solar wind. One of the main outcomes of STORM was a software library of methodologies (e.g., power spectral density analysis, probability distribution functions and multifractals) able to reveal the structure of turbulence (e.g., Bruno \& Telloni 2015; Consolini et al., 2015a, 2015b). Moreover, the analysis aimed to explore the effect of Space Weather also through the investigation of the scaling and multifractal properties of the fluctuations of the geomagnetic indices at solar maximum versus solar minimum (e.g., De Michelis et al., 2015).

The scientific exploitation of existing space plasma data was also supported by the FP7 "Solar and Heliospheric Collisionless Kinetics" (SHOCK) project (2012-2015) aiming to maximize the scientific return of space missions, through the concrete identification of synergies between space plasma modeling and data analysis. Similarly, to the case of the STORM project, within the SHOCK project, the importance of studying the kinetic processes at small length scales and short time scales to properly understand the fundamental processes behind Space Weather was considered.

For further details on the projects in this topic area, the reader is referred to Appendix A (Topic Annex n. 2).

\section{Topic area n. 3: Ionosphere and plasmasphere}

During a geomagnetic storm, relativistic electron precipitation from the radiation belts provokes the exposure of space assets in radiation of increased intensity. The physical mechanisms behind this precipitation are the interaction of several wave modes with resonant electrons leading to the scattering of the latter into the atmospheric loss cone. The interactions between the waves and radiation belt particles influencing the properties of the plasmasphere and the details of the involved physical mechanisms are yet not completely understood. Research work within this direction has been performed within the FP7 "PLASMON: A new, ground-based data-assimilative modeling of the Earth's plasmasphere - a critical contribution to Radiation Belt modeling for Space Weather purposes" project (2011-2014). In particular, a plasmasphere model was developed based on data from the European quasi-Meridional Magnetometer Array (EMMA) and equatorial electron densities derived from a worldwide network of whistler recording stations. This model being continuously fed up with new measurements was used to identify structures inside or outside the plasmapause that are likely to result in enhanced electron losses (the reader is referred also to the papers by Heilig \& Lühr, 2013; Vellante et al., 2014). In this way, relativistic electron precipitation was monitored during periods of high geomagnetic activity.

For further details, the reader is referred to Appendix A (Topic Annex n. 3).

\section{Topic area n. 4: Particle radiation}

Understanding the properties of the intense radiation environment is one of the key science challenges of planetary Space Weather in view of human and robotic exploration in space. An in-depth knowledge of the variability of the radiation conditions at a specific location in the Solar System is an important requirement for the quantified prediction of the dangers of space exploration to machine operations and life (where applicable). Within the FP7 "Environment for Human Exploration and RObotic Experimentation in Space" (e-HEROES) project (2012-2015), data from European and international space missions were exploited to estimate and predict the threats that future exploration missions to planetary bodies may encounter. Special consideration was given to space missions to venture beyond low Earth orbit - to the Moon, Mars, and beyond. The developed models can be used also for estimating how spacecraft may be charged as they pass through solar and planetary magnetic fields. The outputs of this project may be linked to future studies also in the field of Solar System exploration.

Radiation dosimetry is another aspect related to Space Weather and human exploration in space, nevertheless, very few dosimetric data are available in literature at high southern latitudes. Radiation dosimetry campaigns have been performed in the Antarctic region in the framework of the "Cosmic Rays in Antarctica" (CORA) project (2013-2015), a collaboration between Argentine and Italian institutions, aiming to measure the various components of the cosmic ray induced secondary atmospheric radiation at the Argentine Marambio Base (196 $\mathrm{m}$ above sea level).

For further details on the projects in this topic area, the reader is referred to Appendix A (Topic Annex n. 4).

Topic area n. 5: Infrastructure and service development

Research infrastructures and service development projects have always been a major asset for Space Weather. One of the first concrete examples of a research infrastructure benefiting Space Weather science in view of service development was the FP7 "Heliophysics Integrated Observatory" (HELIO) project (2009-2012), which addresses the needs of a broad community of researchers in the field. Through this project, access to data from numerous instruments from observatories throughout the heliosphere was provided (Aboudarham et al., 2012). The services that have been established were used to search for, track and relate events and physical phenomena.

The parallel FP7 "Near-Earth space data infrastructure for e-science" (ESPAS) project (2011-2015) supported the modeling and the prediction of the near-Earth space environment providing access to a large set of databases that have been developed for the needs of different Space Weather users (Belehaki et al., 2016). The easier use of key research infrastructures that had registered their data in ESPAS was one of the major advantages of that project. A representative example is EISCAT, the European Incoherent SCATter Scientific Association, a major European research infrastructure with radar facilities in Northern Scandinavia and at Svalbard. 
In this perspective, an important effort to provide access to both real-time and historical high-resolution neutron monitor measurements from stations of the world-wide network, including the ones of the Rome station (called SVIRCO Observatory; for details, the reader is referred to Sect. 2.2.1) was made in the context of the FP7 "Neutron Monitor Data Base (NMDB)" project (2008-2009). This project evidenced immediately the synergy between different science disciplines (e.g., solar physics, particle radiation physics) required for understanding the entire chain of a Space Weather phenomenon. The NMDB project brought together the cosmic ray community of the European neutron monitor network with the scope to advance the use of cosmic ray data in cutting-edge applications, such as those related to Space Weather (see, for instance, Mavromichalaki et al., 2011 and references therein). Numerous modeling studies and investigations based on cosmic ray data analysis made use of the NMDB database. The study of Space Weather phenomena such as the Forbush decreases (e.g., Belov et al., 2010; Lingri et al., 2016) and the GLE events (e.g., Plainaki et al., 2009a, 2009b, 2014) was significantly facilitated by the existence of NMDB.

In the context of an interdisciplinary approach, Space Weather data from different space-based (PAMELA, ALTEA; see Sect. 2.3.2) and ground-based (IBIS, MOTHII; see Sect. 2.2) instruments were selected within the "Space WEeatherR TOr vergata university" (SWERTO) database (2015-2017; Berrilli et al., 2018). Simulation-based investigations of the evolution of different Space Weather parameters are possible through the efficient use of the SWERTO data.

The time-varying solar spectral irradiance is believed to have a role in climate change hence its record is highly relevant also for Space Weather science. The FP7 "Solar Irradiance Data Exploitation" (SOLID) project (2012-2015) aimed at creating a single homogeneous solar irradiance record out of numerous observations (Haberreiter et al., 2015). A significant advance to current knowledge in the solar variability (from the beginning of the space era to the present) was obtained through the SOLID project. Moreover, the synergies and collaborations between heliospheric and climate science communities were strengthened. Within the SOLID project, two different state-of-the-art models were used to produce the reconstructed spectral and total irradiance data as a function of time. The revised irradiance time series provided by the SOLID project are highly relevant not only for Space Weather science applications but also for disciplines such as the Solar System exploration.

In view of Space Weather forecasting, significant effort has been devoted in the development of the mathematical framework supporting space weather services. One concrete example in this direction is the FP7 "Space weather integrated forecasting framework" (SWIFF) project (2011-2014; Lapenta et al., 2013). One well-known difficulty during the development of data-driven simulations is the existence of plasma parameter variations by many orders of magnitudes going from the Sun to the Earth, in the presence of a wide variety of physical processes with different temporal and spatial scales. Treatment of these problems become very complex in particular at the interfaces between very different plasma regions, such as the photosphere and the corona, or the solar wind the and the magnetosphere: in these regions, therefore, it is mandatory to couple different descriptions of plasma, such as the kinetic, two fluid or single fluid approximations. In this view, the possibility of scientific synergies between this project and efforts within the topic area of space plasmas arise.

One of the major international infrastructures of the next years dedicated to the study of the fundamental processes in the Sun controlling the solar atmosphere, activity, and Space Weather sources, is the next-generation large-aperture European Solar Telescope (EST). Numerous scientific projects have aimed to support the development of such an important ground-based instrument. The conceptual design and feasibility of EST have been studied through the FP7 "EST: The large aperture European Solar Telescope" project (2008-2011), whereas the FP7 "SOLARNET - High-resolution Solar Physics Network" project (2013-2017) promoted the coordination of the major European research infrastructures. Such collaboration allowed synergies and linkages in the field of tool and instrument prototype development related to the future operation of EST. This effort was followed by the H2020 "SOLARNET Integrating High Resolution Solar Physics" project (20192022) that brings together European infrastructures in the field of high-resolution solar physics. In parallel, the H2020 "Getting Ready for the EST" (GREST) (2015-2018) and H2020-PREEST (2017-2021) projects have been providing important feedback in the field of instrumentation.

The work on Space Weather services and operations can favor also the birth of ideas which may influence the definition of science objectives and related observational requirements for future instrumentation. Feedback from operations can be therefore a powerful stimulus to Space Weather science. The COSPAR and ILWS roadmap offered a global framework for such Operations-to-Research (O2R) discussions. Similar O2R activities have been an important aspect also within Space Weather projects in which the Italian community has been involved, allowing science to respond to ideas coming, for example, from Space Weather risk assessments and mitigation plans. A pertinent example is the Ionosphere Prediction Service (IPS) project (2015-2017) of the European Commission in the frame of the Galileo programme (Albanese et al., 2018), where research activities were strongly linked to the provided Space Weather service (Rodriguez et al., 2018). Additionally, the PECASUS (Pan-European Consortium for Aviation Space Weather User Services) initiative aims for a global Space Weather information service center as specified by the International Civil Aviation Organisation (ICAO). Although PECASUS considers solely the operational aspect of Space Weather, aiming to become Europe's leading Space Weather center (resilient 24/7 manned operational), it makes use of an extensive technical and scientific expertise across multiple European institution hence allowing a continuous and coherent feedback exchange between Space Weather science and operations.

The newborn Space Weather service of the Italian Air Force (AMI - Aeronautica Militare Italiana) was developed in the context of its meteorological service. The Space Weather service is composed of a Space Weather Centre, a Backup Centre, and a Training Centre. Starting from 12th of March 2018, the service could rely on an Initial Operational Capability (IOC) level; an observation and a forecast bulletin were issued daily and allocated only to the Institutions of the Italian Defense. A Full Operational Capability (FOC) level was obtained on 7th of January 2019 with the daily emission of four bulletins (i.e., one every 6 h). In the next future, the Italian Air Force will 
provide Space Weather products and briefings to the Italian community (limited to its own institutional tasks) with a widespread dissemination. It is very important to underline that, according to the present Italian Air Force roadmap, both IOC and FOC were and are based, for the moment, on the numerous NOAA's American public products. These American products were structurally synthesized in the current "Italian" military bulletin. The Italian Air Force needs to improve its Space Weather Service moving forward through the two following steps:

- acquire International, more detailed and already operational products through bilateral agreements;

- acquire Italian academic, research Institutes and aerospace companies products, once realized, for a preferred national autonomy.

Finally, important effort has been devoted in the design of telescopes capable of monitoring the solar activity. In particular, within the Solar Activity MOF Monitor (SAMM) project, the possibility to develop a double channel telescope to measure the solar magnetic field has been evaluated. In addition, the Tor Vergata Solar Synoptic Telescope project has been focused on the study of the design of a double telescope to obtain full disk solar dopplergrams and magnetograms. Such a telescope will be part the first Magneto-Optical Filter (MOF)-based network to investigate and automatically detect flare location and associated photospheric features.

For more technical details on the projects in this topic area, the reader is referred to Appendix A (Topic Annex n. 5).

\subsection{Ground-based infrastructures}

Ground-based observations of Space Weather phenomena have long been an important resource for research in the field of solar-terrestrial physics. Even during the space era, to have a complete picture of an ongoing Space Weather phenomenon, it is often necessary to carry out a joint analysis of the observations obtained by both ground-based and space-borne instruments. Numerous studies on the coordinated analysis of data from ESA's Cluster mission, ground-based radar and optical observations provide an example of how a combination of both space- and ground-based data is crucial for resolving temporal and spatial features in the near-Earth space environment (indicatively, the reader is referred to the works by Lockwood et al. (2001) and Opgenoorth et al. (2001)). Moreover, ground-based data analyses very often provide additional support to space missions.

Ground-based infrastructures can be distinguished into ground-based Space Weather assets, providing observations of the temporal and spatial variability of key Space Weather parameters, and facilities for ground testing of space systems.

\subsubsection{Ground-based Space Weather assets}

Italy has been operating an extended set of observational assets that on a regular basis monitor the state of the Sun, the Earth's magnetosphere and ionosphere, and the solar and galactic cosmic ray intensity, providing often near-real-time data relevant to the conditions of the Sun and the geospace. Because of their spatial distribution and qualitative measurements, the ground-based observations obtained by the Italian assets contribute to the international datasets currently available for scientific analysis of Space Weather phenomena. In Figure 3, we provide an indicative map of the main ground-based observing systems showing the coverage provided by Italian Space Weather sensors. As shown in Figure 3, the Italian assets make part of the European and global sensor provision, providing thus a potential mutual benefit in a more global Space Weather context.

$I N A F$ is responsible for the operation of a series of Space Weather assets that monitor the solar emissions in the optical (see Table 2) and radio bands (see Table 3), the ionospheric irregularities by HF radar pulses (see Table 4), and the solar and galactic cosmic ray intensity (see Table 5). At the same time, an extensive number of historical data archives (indicatively, we refer to the Historical Solar Data Archive - HISTO-A; see Table 2), the Interferometric Bidimensional Spectropolarimeter Data Archive (IBIS-A; see Table 2 and Appendix B, Note 1), the SOHO Long-term Archive (SOLAR; contains data from 11 out of 12 SOHO instruments; see Table 2), and the Trieste Solar Radio System 1.0 Data Archive (TSRS1.0-A; see Table 3 and Appendix B, Note 2) are currently maintained and studied. Data from the THEMIS telescope in the Canary Islands are also available to SWICo; such measurements (e.g., exospheric $\mathrm{Na}$ emission patterns at Mercury) can be used for post-event analysis as well as for Planetary Space Weather investigations (e.g., Mangano et al., 2015; Orsini et al., 2018). For a concise overview of technical details considering the actual Space Weather assets operated by INAF and data-archives the reader is referred to Tables 2, 3, 4, and 5, whereas some additional material considering the actual functionality of the assets is provided in Appendix B. Below we provide a brief overview of the capabilities of single Space Weather assets, evidencing - where possible - how they can best contribute to European and global networks.

Full-disk synoptic observations of the Sun are made by different ground-based telescopes, e.g., the $\mathrm{H} \alpha$ and $656.78 \mathrm{~nm}$ continuum telescope in Catania (INAF-OACt), the $\mathrm{Ca} \mathrm{K}$ and continua PSPT telescope in Rome (INAF-OAR), and the K1 D1 line MOF filter VAMOS (Magrì et al., 2008) and the WL photospheric and $\mathrm{H} \alpha$ telescopes in Naples (INAF-OACN). $I N A F-O A C t$ is one of the Expert Groups of the "Solar Weather" Expert Service Center (ESC) of ESA's Space Situation Awareness (SSA) Program. Moreover, using daily observations of the photosphere, INAF-OACt provides an indication of the probabilities that each sunspot group visible on the solar disc may host solar flares of C-, M-, and X-class (see, for instance, in Contarino et al., 2009). For further technical information, the reader is referred to Appendix B, Notes 3, 4, and 5).

Monitoring of the solar radio bursts are carried out by e-Callisto, an international network of solar radio spectrometers. INAF-OATs contributes to e-Callisto with two spectrometers (uncalibrated radio flux density, no polarization; 45-81 MHz band and 220-425 MHz band). Within 2019, the Trieste Solar Radio System 2.0 (TSRS 2.0), an agile solar radio spectropolarimeter operating in the $1-19 \mathrm{GHz}$ band, will start operating. It will provide diachronic near-real-time solar radio data (calibrated radio flux density and accurate circular polarization) for Space Weather applications (Messerotti, 2018) with special attention to the L-band. Furthermore, preliminary solar radio imaging in the K-band by Sardinia (SRT) and Medicina radio telescopes have started (Pellizzoni et al., 2018) and will be 


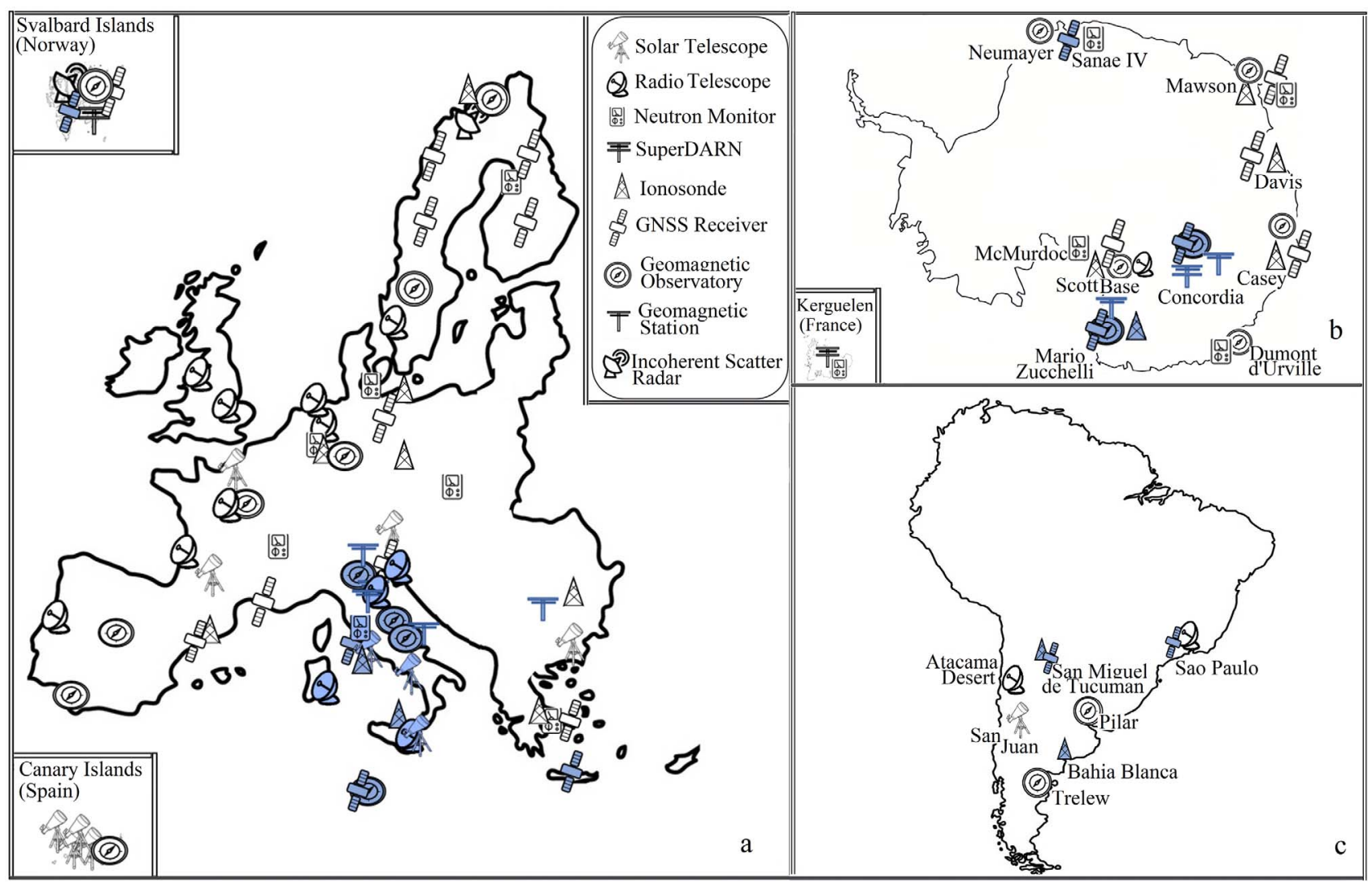

Fig. 3. Indicative map of the main ground-based Space Weather assets showing the coverage provided by Italian Space Weather sensors (shown in blue). The Italian assets make part of the European and global sensor provision, providing thus a potential mutual benefit in a more global Space Weather context. (a) Indicative map of the ground-based Space Weather assets in Europe. (b) and (c) Indicative maps of the ground-based Space Weather assets outside the European territory (in particular, in the Antarctic region and South America, respectively), totally or partially managed by Italian research institutes and universities. Solar emissions in the optical bands are monitored by the H $\alpha$ and $656.78 \mathrm{~nm}$ continuum telescope in Catania (INAF-OACt), PSPT (INAF-OAR), and VAMOS (INAF-OACN) (for details see Table 2). Solar emissions in the radio bands are observed by SunDish, the Italian Single-Dish Radio Telescope network (INAF-OACa, INAF-IRA Bologna and Noto, INAF-OATs, UNICA, UNITS, ASI, ASTRON), TSRS 2.0 (INAF-OATs), and the Trieste Callisto System (INAF-OATs) (for details see Table 3). Ionospheric irregularities are monitored by the Dome C East and Dome C North HF radars in the Antarctic region (INAF-IAPS), part of the SuperDARN network, ionosonde and digisonde instruments in Italy, Argentina and the Antarctic region (managed by INGV), and GNSS receivers in Italy, Europe, Argentina, Brazil and the Antarctic region (managed by INGV) (for details see Tables 4 and 7). Geomagnetic field measurements are registered at the geomagnetic observatories operated by $I N G V$ (four in Italy and two in the Antarctic region), at the four European SEGMA stations, operated by UNIVAQ, and at the two geomagnetic pulsation facilities in the Antarctic region, operated by UNIVAQ (for details see Table 8 and text). Considering the international assets, the information was obtained mainly from following sources: the "COSPAR roadmap for space weather activities: asset catalogue" document (http://www.spaceweathercatalogue.org/COSPARCatalogue.pdf); the "2017 ISWI Workshop" material http://www.unoosa.org/oosa/en/ourwork/icg/activities/2017/iswi-sc2017.html, the INTERMAGNET website (http://www.intermagnet.org/imos/imomap-eng.php\#), the NMDB website (http://www.nmdb.eu/), material within the NOAA website (https://www.swpc.noaa.gov/) and references therein. We underline that the current figure does not depict the entire network of ground-based Space Weather assets, nevertheless, it provides a rough picture of the distribution of the Italian observatories with respect to the main international capabilities in the field.

followed by the Noto Radio Telescope. These non-dedicated radio infrastructures have been collaborating with the LowFrequency Array radio telescope network (LOFAR; e.g., Mann et al., 2018; Reid \& Kontar, 2018) and other radio facilities for Space Weather applications (Messerotti 2019).

INAF-IAPS has the responsibility of the Dome $\mathrm{C}$ East (DCE) and Dome C North (DCN) HF ionospheric radars and manages the two radars in collaboration with $C N R$ and the support of the Italian Program for the Antartic Research. Both radars are part of the Super Dual Auroral Radar Network (SuperDARN) network (Greenwald et al., 1995; Chisham et al., 2007; Lester, 2013) that comprises more than $30 \mathrm{HF}$ radars, all operating continuously, observing the ionosphere from mid-latitudes to the polar regions in both the Southern and Northern hemispheres. DCN and DCE therefore operate in the context of a more global strategy contributing in the international effort to perform a hemispheric characterization of the ionospheric plasma convection, within a global Space Weather 


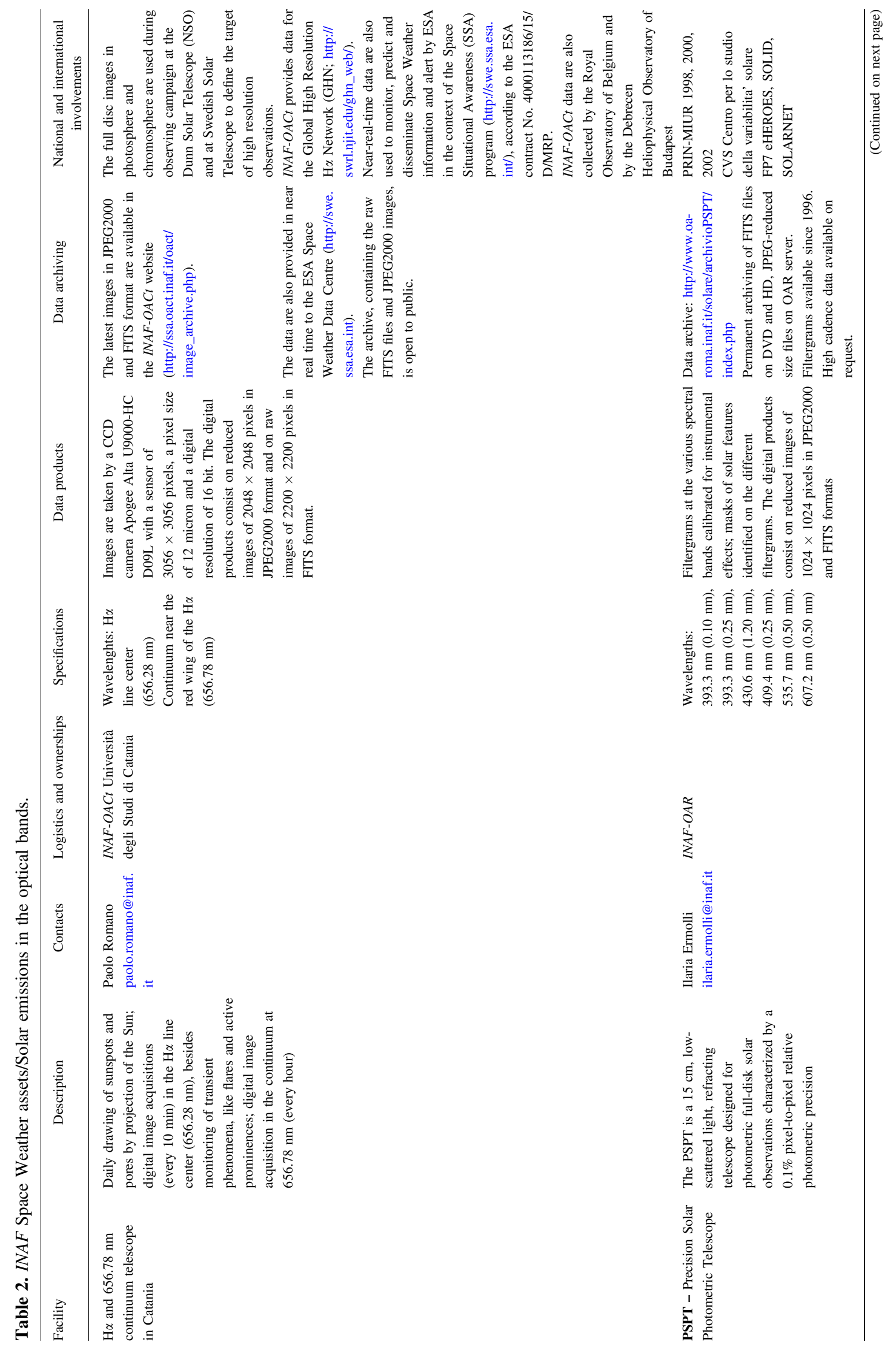




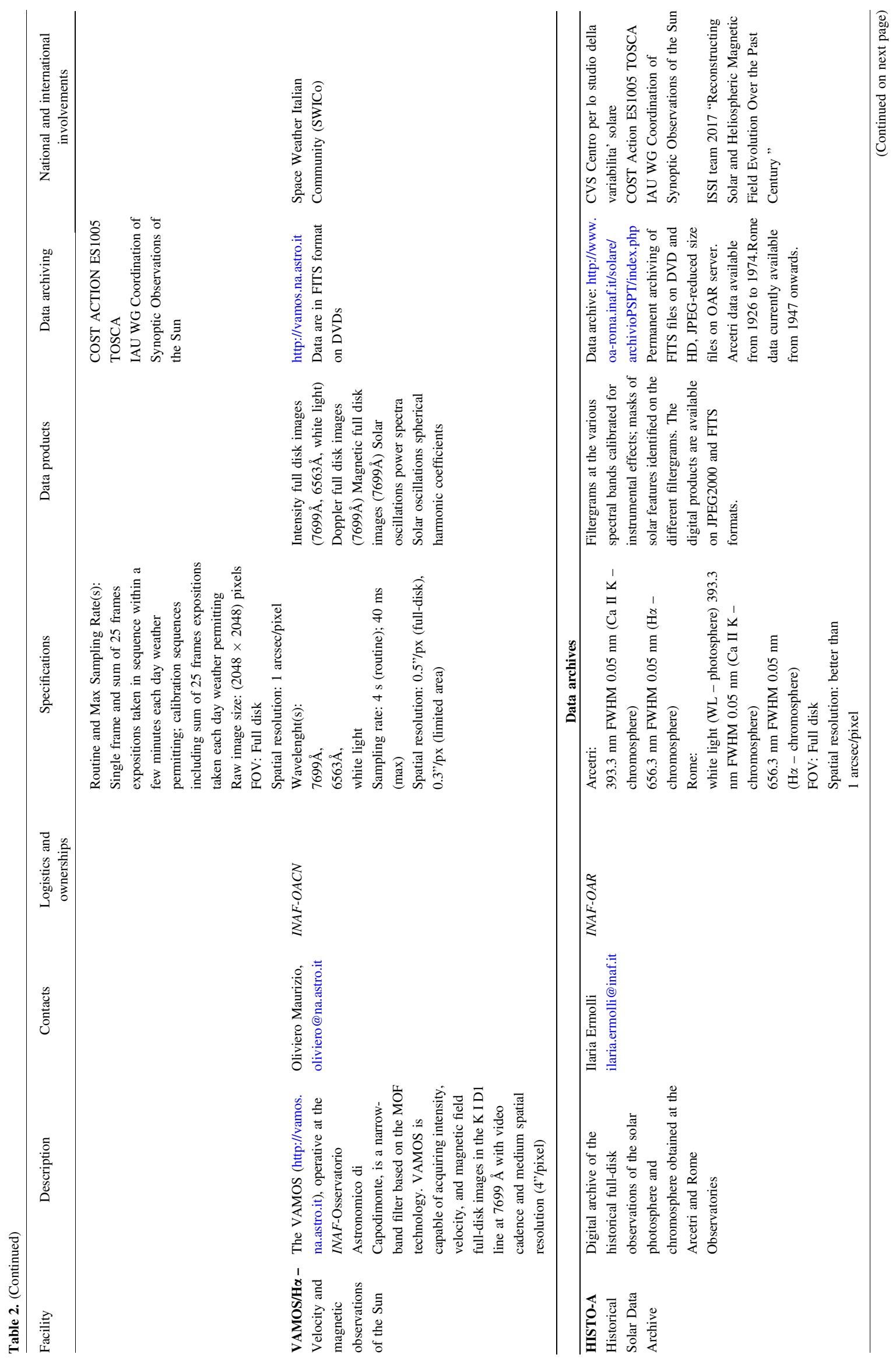

Page 11 of 51 


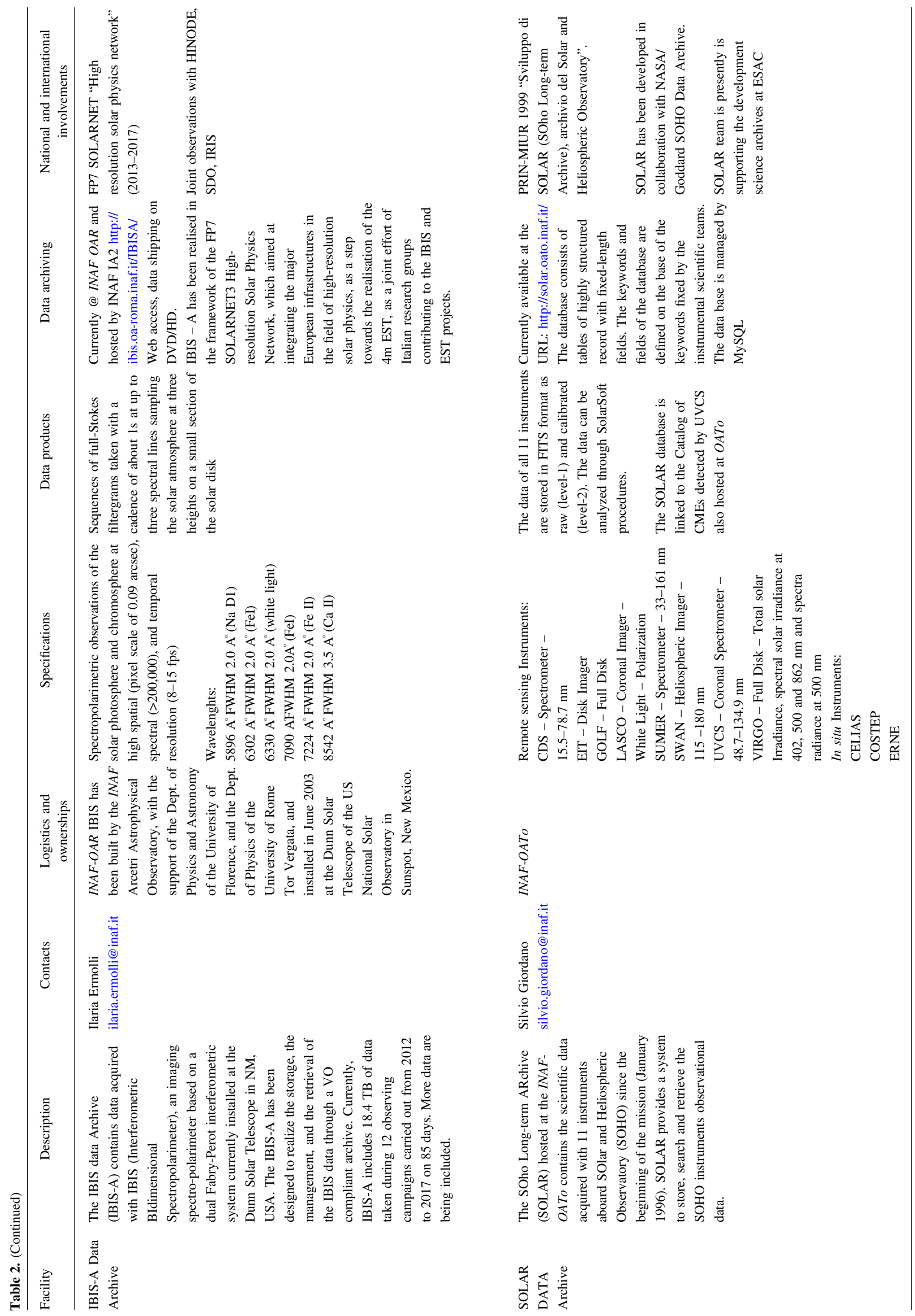




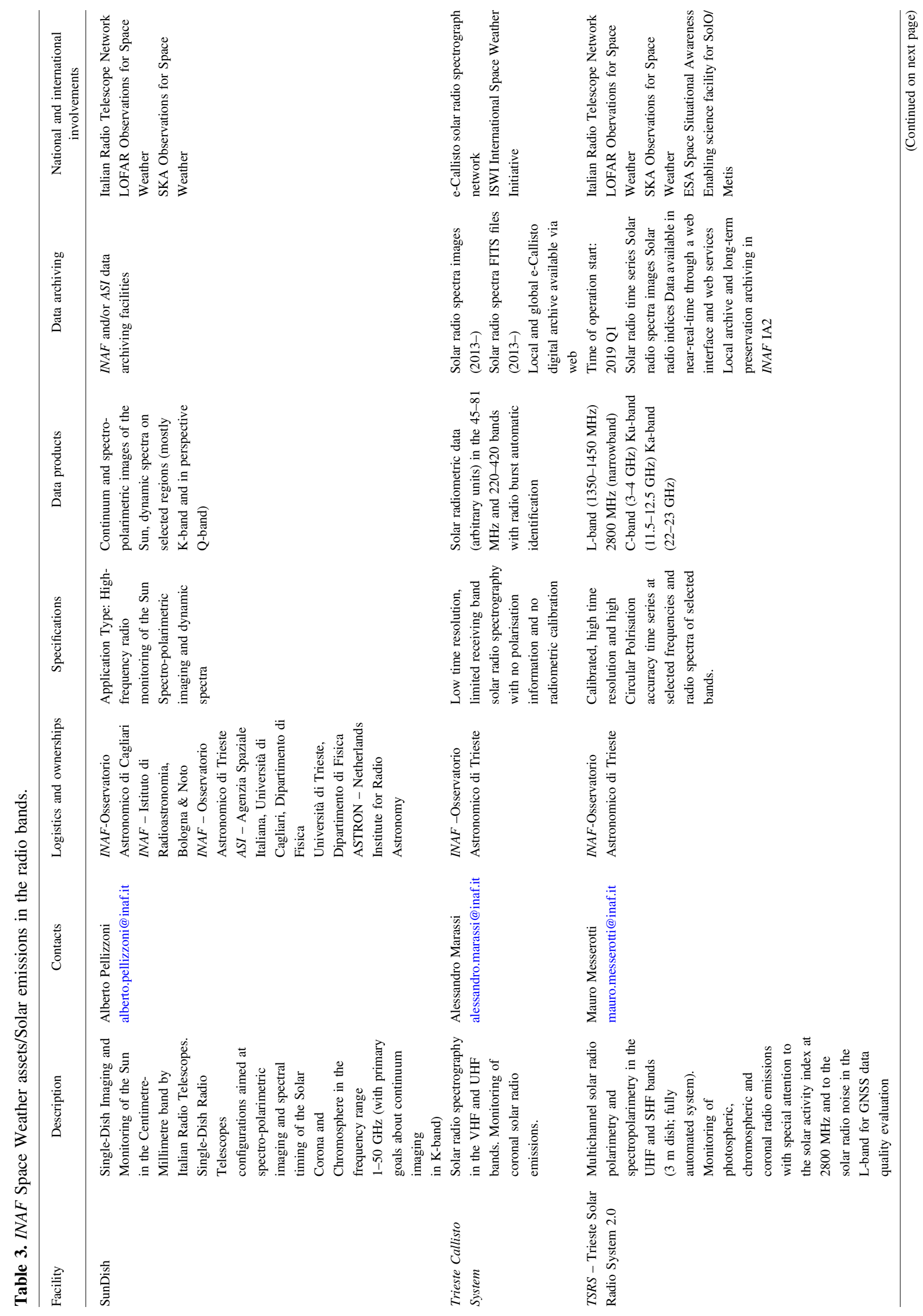




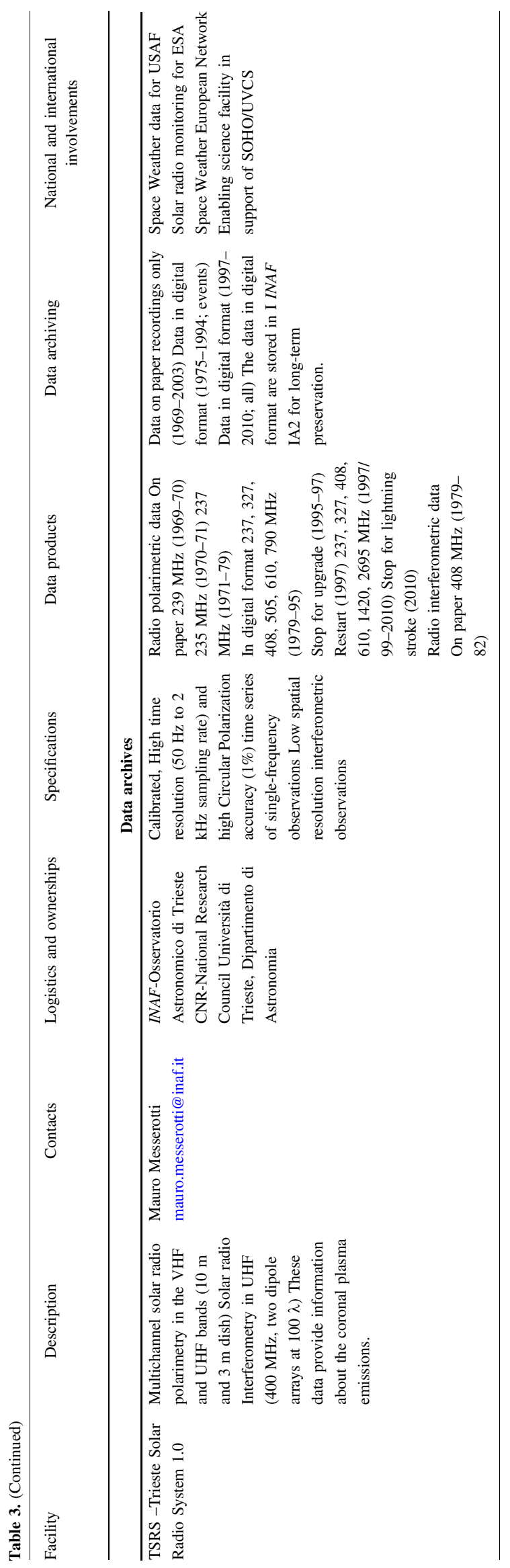

context. SuperDARN and space born observations have been fruitfully and largely used in a coordinated fashion (e.g., with Cluster, IMAGE, THEMIS, Swarm, ARASE) exploiting the highly valued synergy between high resolution measurements in the geospace and the global SuperDARN observations (e.g., Wild et al., 2003; Trattner et al., 2005; Marcucci et al., 2008; De Michelis et al., 2016; Shinbori et al., 2018). Indeed, ground- and space-based observation coordination is very important and, in this context, SuperDARN scheduled campaigns to support several missions, e.g., the ESA Cluster mission, the NASA Magnetospheric Multiscale Mission (MMS) and Van Allen Probes missions, and the ISAS/JAXA/ISEE Exploration of energization and Radiation in Geospace (ERG) mission. The SuperDARN observations are fundamental for Space Weather science, in terms of the study of the magnetosphere-ionosphere coupling with emphasis in the characterization of the global ionospheric convection, Ultra Low Frequency (ULF) waves, field-aligned currents, substorms, ionospheric irregularities and plasma structures (patches) physics. For related technical information, the reader is referred to Appendix B (Note 6). In addition, the All-sky camera for auroral observations located in Ny Alesund (Svalbard) and the Magnetometers - Ionospheric Radars-Allsky Cameras Large Experiment (MIRACLE), lead by the Finnish Meteorological Institute, permit the study of the dayside auroral events connected to the magnetospheric cusp precipitation, also in coordination with other ground- and space-based instrumentation (INAF-IAPS participates in these projects).

In the context of Space Weather, the ground-based counterpart of relativistic SEPs directed to the Earth (i.e., the GLEs) can be registered at ionization chambers, muon detectors, and neutron monitors. The responses of ground-based neutron monitors to SEPs with rigidities bigger than $\sim 1 \mathrm{GV}$, are often used to solve the inverse problem for determining a best-fit spectrum of the primaries (see, for instance, Bombardieri et al., 2007; Plainaki et al., 2007, 2010; Miroshnichenko, 2018 and references therein). The SVIRCO Observatory (INAF-IAPS and UNIRoma3 collaboration) in Rome is equipped with a neutron monitor (NM-64 type) and provides both important feedback for the high-energy tail of the SEP spectrum during relativistic SEP events and the background GCR nucleonic component. The SVIRCO Observatory has been performing continuous measurements of the secondary nucleonic component of GCR and Solar Cosmic Ray (SCR) intensities since July 1957. It is part of the worldwide network of neutron monitors and it is the only facility of this kind existing in Italy, characterized by high efficiency and reliability. SVIRCO is considered to be one of the essential observational sites for research in the fields of cosmic rays and solar-terrestrial physics (Laurenza et al., 2012, 2014; Storini et al., 2015) as well as a crucial asset for Space Weather science and applications. Indeed, its geographical position allows to detect secondaries corresponding to particles at magnetic rigidities bigger than $6 \mathrm{GV}$ which are crucial for determining the slope of the cosmic ray spectrum. SVIRCO contributes to the real-time database for highresolution neutron monitor measurements (i.e., the NMDB; see also Sect. 2.1) and is a data provider for the ESA SSA Space Weather segment. In addition, the INAF-IAPS synergies include other four neutron monitors (Testa Grigia - Italy; LARC, King George Island - Antarctic; ESO, Mt. Hermon - Israel; OLC, Los Cerrillos - Santiago of Chile), three of them operated with 


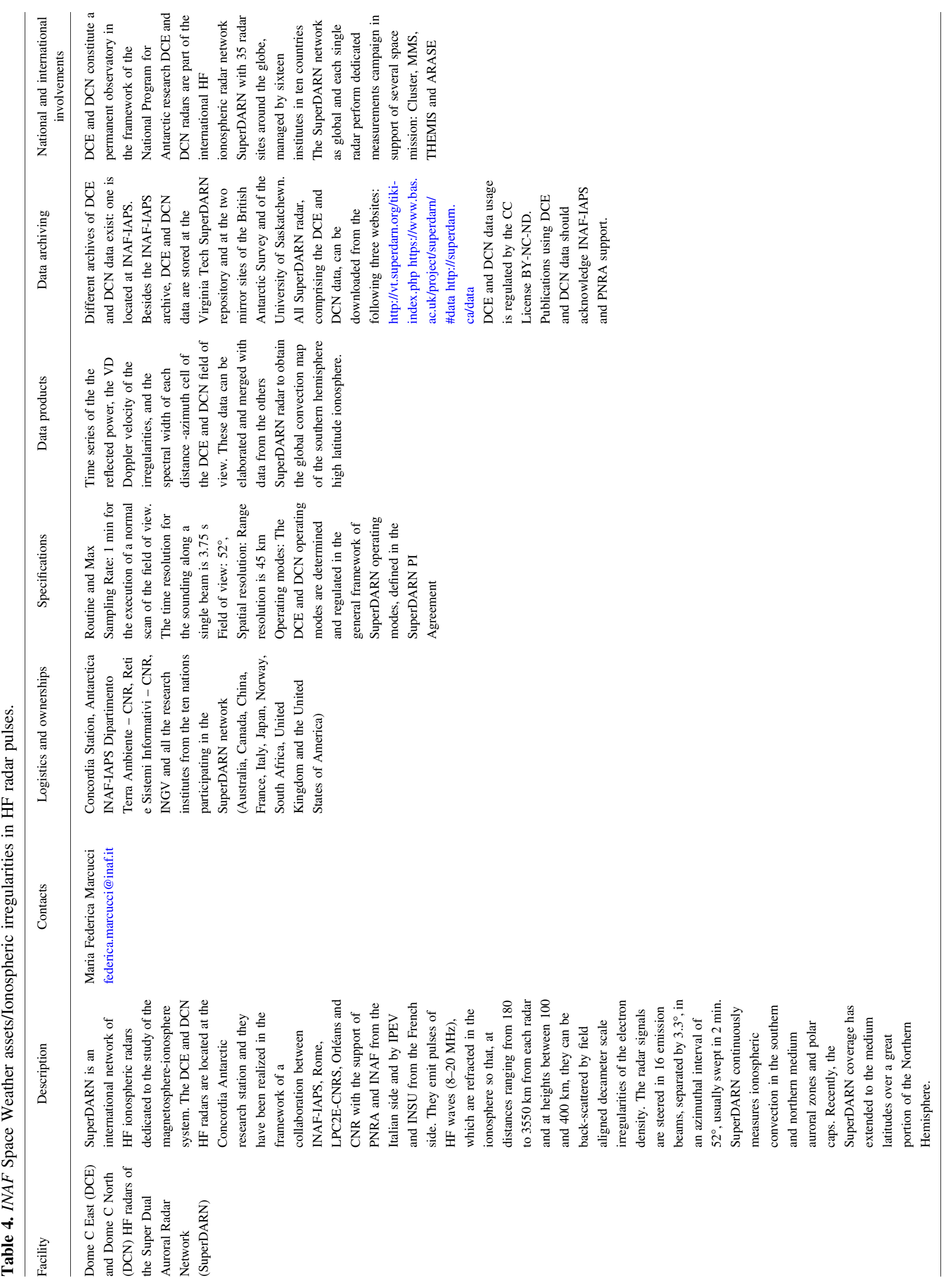




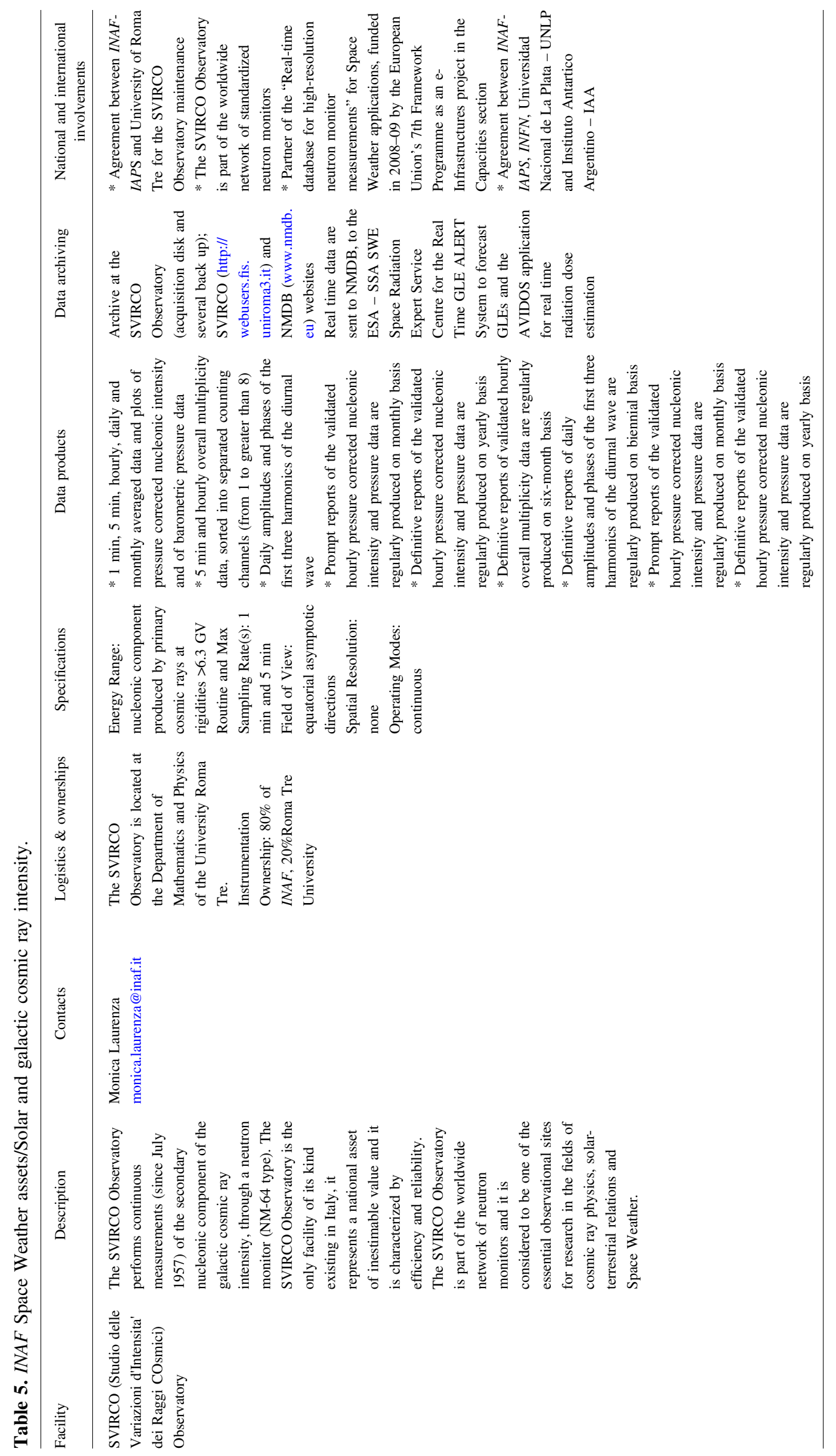


international partners. The four detectors constitute a mini network within the worldwide network of cosmic ray detectors, able to provide a prompt response to the flux variabilities of protons with energies bigger than $3 \mathrm{GeV}$.

The INGV operates three ionospheric stations (in Rome, in the Observatory of Gibilmanna, near Palermo, and at Terra Nova Bay in the Italian base in the Antarctic region). These stations are part of the worldwide network aiming to study phenomena in the Earth's ionosphere. Moreover, thanks to a scientific collaboration between Italy and Argentina, also two Argentinean ionospheric observatories (i.e., Tucumán and Bahia Blanca) are equipped with an Advanced Ionospheric Sounder (AIS) ionosonde, developed by INGV (Alfonsi et al., 2013) To study possible Space Weather impacts on the upper atmosphere, the INGV manages also several GNSS-ionospheric stations (see Fig. 3). The Total Electron Content (TEC) and ionospheric scintillations are measured in Italy, Greece, Svalbard, the Antarctic region, and South America (De Franceschi et al., 2006; Romano et al., 2013; Alfonsi et al., 2016). By means of such a network, $I N G V$ monitors continuously the ionospheric conditions for HF communication to provide reliable GNSS services in Italy and the Mediterranean area, at high and low latitudes. INGV contributes with its ionospheric data and tools to two recent European Space Weather consortiums and projects, namely PECASUS and IPS (see Sect. 2.1), maximizing the overall European input to wider Space Weather issues.

$I N G V$ is also responsible for the monitoring of the Earth's magnetic field variations and absolute intensity in Italy and for the preparation and validation of data from the Italian magnetic observatories (i.e., L'Aquila, Castello Tesino, Lampedusa, and Duronia) and from the Antarctic region observatories located at the Mario Zucchelli Station (Terra Nova Bay) and on the Antarctic plateau at the Concordia Station (Dome C). For some observatories, K-index ${ }^{1}$ values are also available. For further technical information, the reader is referred to Appendix B, note 7). INGV has also a variometer station in Sicily (Gagliano). The capabilities of variometers differ from those of the observatory instruments since the related data cannot be used for determining standard geomagnetic indices of activity or for studying geomagnetic changes within the Earth. However, in terms of Space Weather science and services, both variometers and observatory instruments have their usefulness.

The Solar-Terrestrial and Space Physics Group of UNIVAQ operates the South European Geomagnetic Array (SEGMA), a network of four stations which continuously records geomagnetic field variations at $1 \mathrm{~Hz}$ sampling rate. The group also runs two geomagnetic pulsation facilities in the Antarctic region (one at the Mario Zucchelli Station and the other at the Concordia Station). The main objective of these measurements is to monitor dynamical processes occurring in the Earth's magnetosphere and to provide ground support to space missions. The group also operates a near real-time monitoring system of the magnetospheric plasma mass density between 1.6 and 6.2 Earth radii. For further technical information, the reader is referred to Appendix B, Note 8 .

We note that some of the ground-based Space Weather assets with important Italian contributions are managed and/or

\footnotetext{
${ }^{1} \mathrm{~K}$-index is a three-hourly quasi-logarithmic local measure on a scale of $0-9$ of the maximum disturbance recorded along the horizontal components of the magnetic field.
}

coordinated within international collaborations, maximizing the respective contributions to wider Space Weather issues. Indicatively, we refer to the double channel telescope MOTHII at the South Pole Solar Observatory (SPSO), located at Amundsen - Scott South Pole Station, realized in partnership between the Georgia State University, the Institute for Astronomy - University of Hawaii, and NASA JPL with the support of UNITOV team and sponsored by the National Science Foundation's Division of Polar Programs. The produced solar full disk dopplergrams and magnetograms at two heights of the photospheric-chromospheric region of the Sun's atmosphere, enable the analysis of the dynamics of the plasma and the magnetic field with unprecedented time resolutions paving the way to a new flare forecasting algorithms and for new analysis tools with important space weather applications (Forte et al., 2018). For further technical information, the reader is referred to Appendix B, Note 9. In addition, a solar coronagraph, installed at Concordia in 2018 by INAF-OATo as part of the ItalianFrench Extreme Solar Coronagraphy Antarctic Program Experiment (ESCAPE), will provide the first long-term coronal magnetic field monitoring. The science goal of these observations is to map the topology and dynamics of the magnetic field in corona in order to address questions on the coronal heating and the driving mechanisms of Space Weather, for example through the investigation of the coronal magnetic conditions connected with the initiation of CMEs. For further technical information, the reader is referred to Appendix B, Note 10.

\subsubsection{Ground testing of space systems}

The Space Weather Italian community is enforced also by an intense experimental activity performed with the use of facilities. Such facilities are devoted to the simulating the conditions that instruments will encounter in space (the so called "lab simulation") and to the supporting of the development of instruments with Space Weather science objectives, according to space development standards (e.g., the standards of the European Cooperation for Space Standardization ${ }^{2}$ ). Such activities include instrumental design, prototype development, cooperation with space industry, and test and calibration for the instrument engineering, qualification, and flight models (see also Table 6).

Moreover, there is a strong expertise in the development of optical instruments for space applications and optical components; facilities for the optical characterization and calibration of components, in the range from the Extreme Ultraviolet (EUV) up to Infrared (IR), are also available in Italy (e.g., UNIPD, $C N R-I F N$ ) and have been largely used during the instrument development phases of several missions (e.g., BepiColombo, Solar Orbiter).

\subsection{Payload with science objectives related to Space Weather, developed under Italian leadership or with significant contribution by Italy}

Space-based observations are of fundamental importance for addressing high priority science issues to mitigate the related risks of Space Weather impacts on technology, infrastructure,

\footnotetext{
2 https://ecss.nl/standard/ecss-e-st-32c-rev-1-structural-generalrequirements/
} 
Table 6. INAF ground-based facilities for space instrument development, testing, and calibrations.

\begin{tabular}{|c|c|c|c|c|c|}
\hline Facility & Description & Contacts & Location and ownership & Specifications & Available tests \\
\hline $\begin{array}{l}\text { Plasma Chamber } \\
\text { SWIPS (Solar } \\
\text { Wind and } \\
\text { Ionospheric } \\
\text { Plasma Simulator) }\end{array}$ & 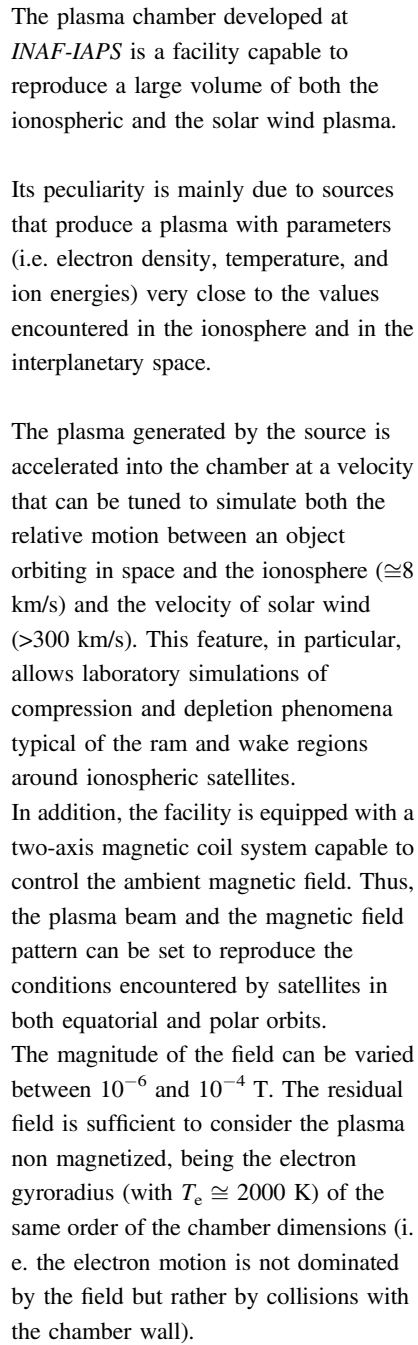 & $\begin{array}{l}\text { Piero Diego } \\
\text { monica.laurenza@inaf.it; } \\
\text { piero.diego@inaf.it }\end{array}$ & $\begin{array}{l}\text { The SWIPS plasma chamber } \\
\text { is an } I N A F \text { facility and it is } \\
\text { located in the experimental } \\
\text { building of } I N A F-I A P S \text {, in } \\
\text { Rome, Via del Fosso del } \\
\text { Cavaliere } 100 .\end{array}$ & $\begin{array}{l}\text { Experimental } \\
\text { volume } \approx 9 \mathrm{~m}^{3} \\
\text { Ionospheric plasma } \\
\text { features: } \\
m_{\mathrm{i}}=40 \text { a.u. (Ar) } \\
T_{\mathrm{e}}=1000-3000 \mathrm{~K} \\
n_{\mathrm{e}} \approx n_{\mathrm{i}} \approx 10^{11}-10^{12} \mathrm{~m}^{-3} \\
v_{\mathrm{i}} \approx 8 \mathrm{~km} / \mathrm{s} \\
\text { Solar wind plasma } \\
\text { features: } \\
m_{\mathrm{i}}=4 \text { a.u. }(\mathrm{He}), \\
T_{\mathrm{e}}=10,000-20,000 \mathrm{~K}, \\
n_{\mathrm{e}} \approx n_{\mathrm{i}} \approx 10^{7} \mathrm{~m}^{-3}, \\
v_{\mathrm{i}} \approx 330 \mathrm{~km} / \mathrm{s}\end{array}$ & $\begin{array}{l}1 \text { - calibration of plasma } \\
\text { diagnostic sensors (Langmuir } \\
\text { probes, Retarding Potential } \\
\text { Analyzer, ...); } \\
2 \text { - functional tests of experiments } \\
\text { envisaged to operate in a } \\
\text { ionospheric environment (sensors } \\
\text { exposed to space plasma); } \\
3 \text { - characterization and } \\
\text { compatibility tests of components } \\
\text { for space applications (materials, } \\
\text { satellite paints, photo-voltaic } \\
\text { cells, etc.); } \\
4 \text { - basic plasma physics } \\
\text { experiments (interaction of } \\
\text { charged bodies with plasma, two } \\
\text { plasma interaction processes, } \\
\text { propulsion and power generation } \\
\text { in space through electrodynamic } \\
\text { tethers); } \\
5 \text { - tests on active experiments } \\
\text { which use cathodes and/or plasma } \\
\text { sources (ion thruster, ion beam } \\
\text { neutralizers, hollow cathodes, } \\
\text { field effect emitters, plasma } \\
\text { contactors). }\end{array}$ \\
\hline
\end{tabular}

and human activities. Italian teams have been often involved in space missions with science objectives related to Space Weather, often with lead roles, also in terms of experiment PI-ships. In Figure 4, the main missions with science objectives related to circumterrestrial and planetary Space Weather, in which there is a significant Italian participation, are presented. The role of the national space industry in the supply of scientific instruments and components for Solar System exploration missions is fundamental for the overall progress in the field of circumterrestrial and planetary Space Weather.

\subsubsection{Space-based instrumentation for the observation of the solar atmosphere}

The Sun is the dominant source of Space Weather in the inner Solar System. Over the last decades, the Italian scientific community acquired an important role in the development of instruments for the observations of the solar corona. For the ESA/NASA SOHO mission (the SOlar and Heliospheric Observatory, launched in December 1995 and still active) the
UV Coronagraph Spectrometer (UVCS, Italian Co-PI-ship) was developed in a collaboration at national level between many different research Institutes (see Table 9) with the fundamental support of the national industry (Leonardo). The UVCS instrument (switched off in 2012) provided tremendous new discoveries on the coronal heating problem, the solar wind acceleration, and the CME evolution (Antonucci, 1994; Susino et al., 2013; Susino \& Bemporad, 2016).

Later on, under the NASA Sounding Rocket Program, the Sounding rocket COronagraphic Experiment (SCORE; Italian PI-ship) was developed again in a collaboration among different Italian research institutes (see Table 9) for the HERSCHEL mission (NASA PI-ship). SCORE is a 3-channel coronagraph onboard the sub-orbital mission HERSCHEL, designed for imaging the solar corona, between 1.5 and 3.5 solar radii, in polarized, broad white-light spectral band, and in the $\mathrm{HI}$ $1216 \AA$ and HeII $304 \AA$ spectral lines. INAF-OATo designed and developed the coronagraph with the innovative concept of multi-wavelength coating for the telescope mirror. Thanks to this innovation, the telescope can simultaneously image the 


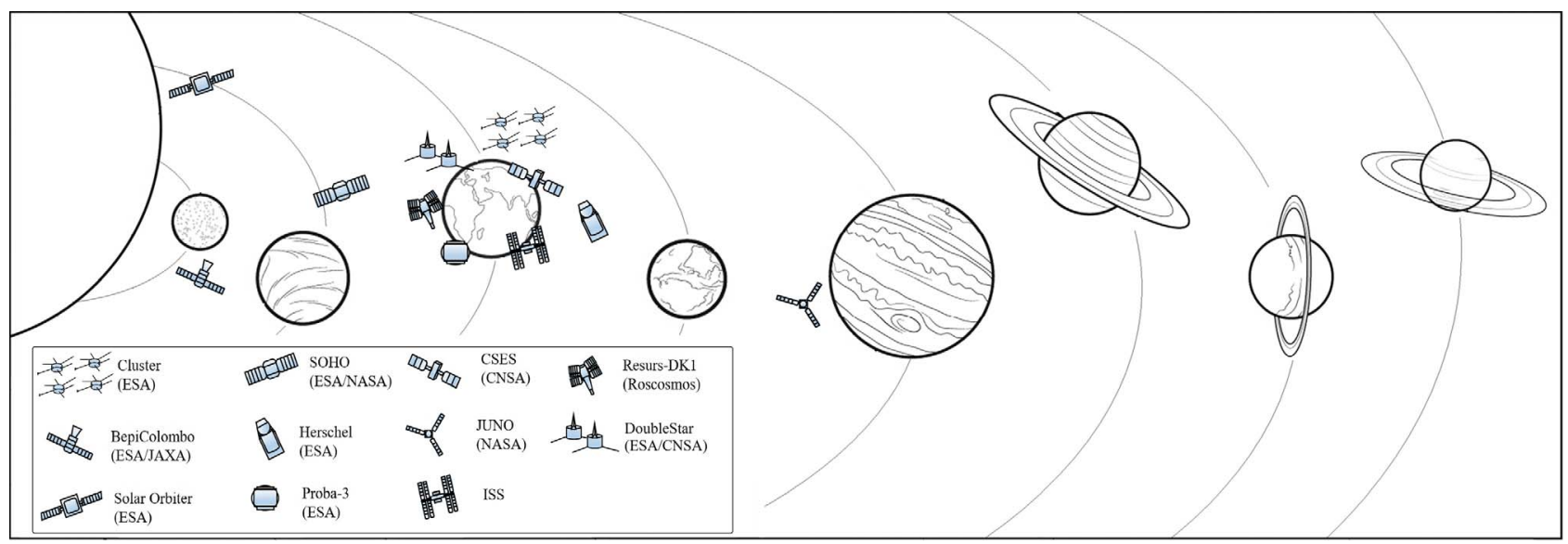

Fig. 4. Graphical view of the main recent, ongoing, and upcoming space missions with science objectives related to circumterrestrial and planetary Space Weather, in which there is a significant Italian participation. The reader is referred also to Table 9.

Table 7. List of ground-based ionospheric observations managed by INGV at different latitudes.

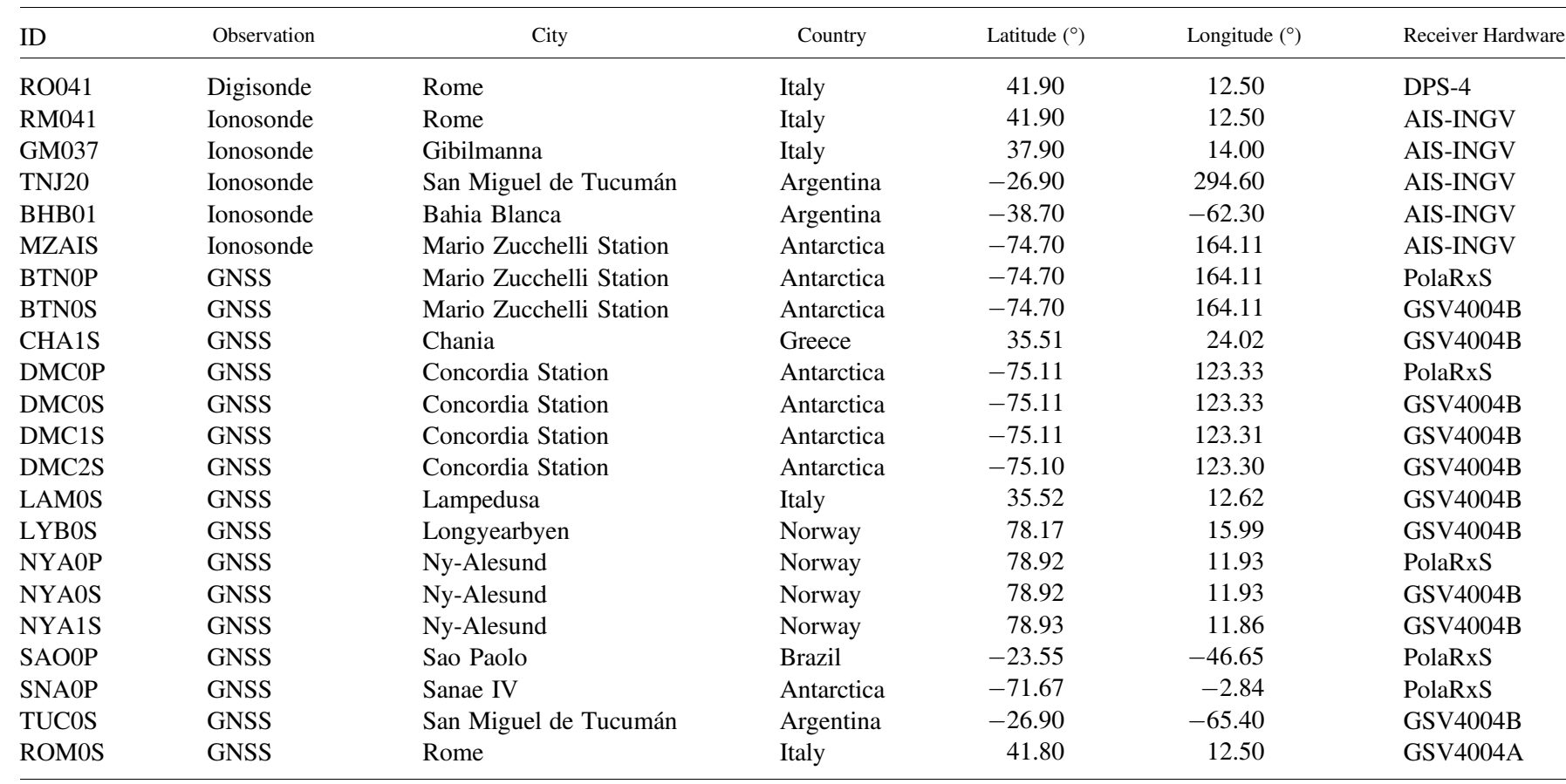

Data and products are accessible at http://www.eswua.ingv.it/ingv and http://ionos.ingv.it/ionoring/ionoring.htm

coronal emissions from coronal free electrons, neutral hydrogen and singly ionized helium ions. The science objective of SCORE is the diagnosis of the mechanisms of energy deposition in the corona and in the solar wind. SCORE is also a mission for Space Weather enabling science. To this aim, following the first successful launch of HERSCHEL on 14th September 2009, SCORE has acquired the first coronal map of helium abundance. The data have shown a strong correlation between the singly ionized helium ions and the magnetic structures at the hole-streamer interface where the solar wind is believed to originate. The SCORE coronagraph acquired successfully the first ever contemporary images of the solar corona in visible light (VL) and in UV with two different narrow band-passes centered around emission from neutral $\mathrm{H}$ atoms (HI Ly- $\alpha$ $\lambda 1216 \AA$ line) and from $\mathrm{He}^{+}$ions (HeII $\lambda 304 \AA$ line).

SCORE was also the proof-of-concept for the Metis coronagraph (Italian PI-ship) developed for the ESA Solar Orbiter mission (instrument delivered in May 2018, now successfully integrated on the spacecraft to be launched in February 2020). Metis (see Table 9) will be the first ever multi-channel coronagraph and will observe the corona in VL (total and polarized brightness) and UV (HI Ly- $\alpha \lambda 1216 \AA$ line), thus providing important information not only on plasma densities (from VL), but also on plasma temperatures and 
Table 8. List of ground-based magnetic facilities of both INGV and L'Aquila University.

\begin{tabular}{|c|c|c|c|}
\hline & IAGA code & Latitude & Longitude \\
\hline \multicolumn{4}{|c|}{ INGV Geomagnetic Observatory } \\
\hline Castello Tesino & CTS & $46.05^{\circ} \mathrm{N}$ & $11.65^{\circ} \mathrm{E}$ \\
\hline Duronia & DUR & $41.65^{\circ} \mathrm{N}$ & $14.47^{\circ} \mathrm{E}$ \\
\hline Lampedusa & LMP & $35.52^{\circ} \mathrm{N}$ & $12.53^{\circ} \mathrm{E}$ \\
\hline \multicolumn{4}{|c|}{ L'Aquila University SEGMA Stations } \\
\hline L'Aquila & AQU & $42.38^{\circ} \mathrm{N}$ & $13.32^{\circ} \mathrm{E}$ \\
\hline Panagyurishte (Bulgaria) & PAG & $42.51^{\circ} \mathrm{N}$ & $24.18^{\circ} \mathrm{E}$ \\
\hline \multicolumn{4}{|c|}{ Antarctic INGV Geomagnetic Observatory and L'Aquila University station } \\
\hline Mario Zucchelli Station & TNB & $74.70^{\circ} \mathrm{S}$ & $164.1^{\circ} \mathrm{E}$ \\
\hline Concordia Station & $\mathrm{DMC}$ & $75.10^{\circ} \mathrm{S}$ & $123.35^{\circ} \mathrm{E}$ \\
\hline
\end{tabular}

Data and products are held (respectively) at http://roma2.rm.ingv.it/en/facilities/geomagnetic_observatories and http://plasmonserver.aquila. infn.it/EMMA_FLR_DENSITY, respectively.

outflow speed (from UV). The Metis instrument (Antonucci et al., 2000, 2019; Romoli et al., 2017) also demonstrated for the first time the possibility to build an inverted-occulter coronagraph, a new configuration that was conceived for the Solar Orbiter mission (that will approach the Sun at 0.28 AU) to limit as more as possible the amount of light (hence thermal load) at the entrance pupil. METIS was developed with the fundamental support of the industry (Thales Alenia Space Italia; OHB Italia).

The Italian community is also involved in the forthcoming challenging ESA PROBA-3 mission (see Table 9), carrying again a coronagraph on board (ASPIICS, Belgian PI-ship). This mission (foreseen launch in 2021) will test the capability to perform the first ever coronagraphic observations in artificial eclipse condition: one spacecraft will carry the occulter for the second spacecraft at a distance of $\sim 150 \mathrm{~m}$ hosting the coronagraph; observations of the inner VL corona will be provided by maintaining the two spacecraft in formation-flying configuration, with a relative precision by $\sim 0.5 \mathrm{~mm}$. Italian teams have been involved in the design of the ASPIICS instrument (Renotte et al., 2015).

The Extreme UltraViolet Spectroscopic Telescope (EUVST) spectrometer, on board the future Solar-C mission of $J A X A$, will observe the solar atmosphere (from the chromosphere up to the corona) in the wavelength range from 170 to $1300 \AA$ with seamless temperature coverage. The mission's scientific objectives are very relevant to Space Weather science. In particular, the mission aims: to understand how fundamental processes lead to the formation of the solar atmosphere and the solar wind; and to understand how the solar atmosphere becomes unstable, releasing the energy that drives solar flares and eruptions. The Italian contribution to this mission is twofold: a) scientific expertise in the design and development of the instrument as well in the exploitation of the data, tapping from a strong tradition in UV and EUV spectroscopy $(O A C N)$; and b) a slit assembly, a key technically challenging component of the spectrometer, feeding both the grating and the slit-jaw imaging system(UNIPD; CNR). The Solar-C mission has been recently selected by $J A X A$, together with two other proposals, for the next phase of substantial studies (corresponding approximately to Phase A). This study phase will end in December 2020 with a down-selection to one mission for a 2024 launch slot with the Epsilon rocket vehicle.

\subsubsection{Charged particle detectors}

Charged particle populations across a broad energy range may be responsible for Space Weather effects on satellites and aviation (Zheng et al., 2019), in either direct (e.g., during SEP or geomagnetic/ionospheric storm events) or indirect (e.g., influencing the background conditions) ways. In the circumterrestrial space, charged particles originate from different sources that are internal or external to the Earth system: several plasma populations extending to suprathermal energies, trapped energetic electrons and inner belt protons/ions, SEPs, and GCRs. Both plasma and energetic particle measurements are a fundamental aspect of Space Weather science. In general, an important constraint for the design of an instrument measuring charged particles at different energies is imposed by the accuracy required to answer a specific science question.

A strong expertise in design, construction, operation and data analysis of particle detectors for radiation measurements in space has been acquired in Italy within a large variety of international programs, jointly supported by $A S I, I N F N$ and Universities. Large use of silicon detectors, implemented with different technologies and integrated with other detectors/ material, has been made in the last 20 years aiming to monitor radiation on board the International Space Station (ISS), to study the interaction of the cosmic radiation with the astronauts brain function and vision system, to measure the GCR composition and flux, and to study proton and electron flux variability at $500 \mathrm{~km}$ altitude in relation with perturbations of the ionosphere/magnetosphere environment. The related experiments have provided or will provide unique data, in different time intervals and/or complementary energy ranges, relevant for the study of the radiation environment in LEO and its variability hence they are particularly important in a wider Space Weather context. Below, we discuss briefly the experiments with science objectives most relevant to Space Weather science. 


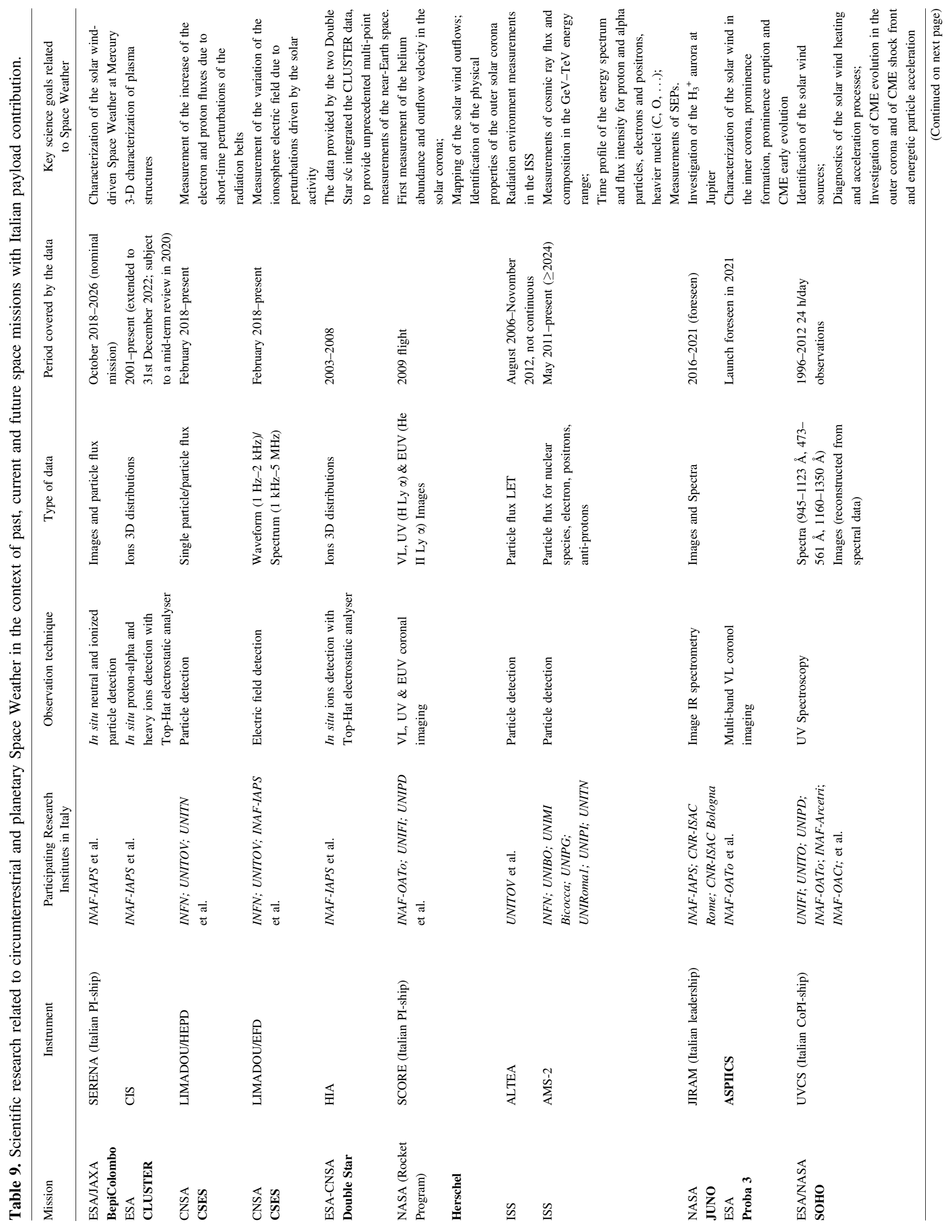


The Anomalous Long Term Effects on Astronauts (ALTEA) program of $A S I$ was devoted to the radiation monitoring onboard the ISS aiming to study the radiation environment in which the astronauts are likely to be exposed during deep space exploration. The ALTEA program, leaded by UNITOV with the fundamental support of the industry (Thales Alenia Space Italia), includes also ground-experiments dedicated to the detailed study of radiation effects on living organisms. Radiation data have been acquired with the ALTEA detector system in the ISS from 2006 to 2012 (Casolino et al., 2006; La Tessa et al., 2009; Zaconte et al., 2010; Larosa et al., 2011; Narici et al., 2012, 2015) and they are of significant importance for future research in the field of Space Weather, especially in the context of human space exploration. Within this program $A S I$ is now sponsoring the Light Ion Detector for ALTEA system (LIDAL), an upgrade of the ALTEA detector system, which will provide an even better ability to study the effect of solar particle events on a LEO habitat (Rizzo et al., 2018). The LIDAL project is lead by UNITOV with the fundamental support of the industry (Kayser Italia).

The Payload for Antimatter Matter Exploration and Lightnuclei Astrophysics (PAMELA) experiment, an ASI/INFN spaceborne satellite for cosmic ray direct measurements built around a magnetic spectrometer, acquired data from June 2006 to January 2016, focusing mostly on antimatter/matter identification and on SEP studies (see, for instance, Adriani et al., 2015; Bruno et al., 2018 and references therein). With an energy interval ranging from $80 \mathrm{MeV}$ up to $1 \mathrm{TeV}$, PAMELA filled the gap between the in situ SEP flux measurements and their counterpart on the ground (registered by neutron monitors), measuring with good accuracy the spectral features (at moderate and high energies), and anisotropies in solar particles arrival directions. In a wider Space Weather context, the PAMELA observations allowed the investigation of the relationship between low- and high-energy particles, providing insights in the SEP origin (Bruno et al., 2018).

The Alpha Magnetic Spectrometer (AMS-02), operates on the ISS since May 2011 to search for anti-matter signals and to study the chemical composition and energy spectra of charged cosmic rays up to $\mathrm{Fe}$, with unprecedented accuracy and in a wide energy range, from few hundreds of $\mathrm{MeV}$ to TeVs. ASI and INFN jointly supported the detector construction, and currently contribute to operation and data analysis activities. AMS-02, developed with the fundamental support of the industry (G\&A Engineering, OHB Italia, and CAEN) and of FBK, is foreseen to operate for the entire life of the ISS, currently at least until 2024. The large acceptance of the instrument $\left(\approx 0.5 \mathrm{~m}^{2} \mathrm{sr}\right)$ and the experiment long duration allow to perform the study of both short- and long-term effects of solar activity on different species of charged particles: measurements of protons, $\alpha$, and heavier nuclei (e.g., $\mathrm{C}, \mathrm{O}$ ) as well as of electrons, positrons, anti-protons are available simultaneously from the same instrument as a function of time, i.e., as modulated by the changing heliosphere conditions, and in an energy range complementary to other missions (e.g., the Cosmic Ray Isotope Spectrometer (CRIS) onboard the Advanced Composition Explorer (ACE) spacecraft or the Electron Proton and Helium Instrument (EPHIN) onboard SOHO). Daily variation of the most abundant species $(\mathrm{p}, \alpha)$ and high-energy SEPs are also being studied. Thanks to the new-generation experiments, such as AMS-02 
and PAMELA, the current solar cycle has been monitored with an unprecedented coverage in terms of multi-channel and timeresolved measurements on cosmic-ray leptons and nuclei (Adriani et al., 2016; Bindi et al., 2017; Aguilar et al., 2018a, 2018b; Martucci et al., 2018). The recent release of these data has generated widespread interest in both the astrophysical and Space Weather communities, leading to substantial advance in the theoretical understanding of the GCR transport and modulation in the heliosphere (Usoskin et al., 2017; Tomassetti et al., 2017, 2018; Aslam et al., 2019; Corti et al., 2019).

The China Seismo-Electromagnetic Satellite (CSES) is part of a collaboration program between the China National Space Administration (CNSA) and ASI. The CSES satellite aims to monitor electromagnetic, particle and plasma perturbations in the magnetosphere, inner Van Allen radiation belts and ionosphere, originating from electromagnetic sources external and internal to the geomagnetic cavity, cosmic rays and solar events. In particular, the objective of the mission is to investigate lithosphere-atmosphere-ionosphere coupling mechanisms (including effects of lightning, earthquakes, volcanoes and artificial electromagnetic emissions) that induce perturbations of the top side of the ionosphere and lower boundary of the radiation belts. To this purpose, the mission has been conceived to take advantage of a multi-instrument payload comprising nine detectors for the measurement of electromagnetic field components, plasma parameters and energetic particles, as well as X-ray flux. The Italian team participating in the CSES mission has built one of these devices, the High Energy Particle Detector (HEPD), for high-precision observations of electrons, protons and light nuclei. Moreover, the Italian team participated in the development of the Electric Field Detector (EFD). Both instruments were developed with the fundamental contribution of Italian Small- and Medium-sized Enterprises (SMEs). During the trip along the orbit, and thanks to the large set of detectors operated on board, CSES acts as an excellent monitor for Space Weather phenomena. The satellite was launched on 2nd February 2018, with an expected lifespan of 5 years.

The Italian scientific community has been participating in the Cluster II Cornerstone mission of the ESA's Space Science Horizon 2000 programme (Escoubet et al., 2001). In the context of Space Weather science, Cluster II is a key mission, being dedicated to the study of the Sun-Earth relationship starting from the in situ three-dimensional investigation of the smallscale plasma structures at the origin of Space Weather phenomena. Italy contributed to the development of the Cluster Ion Spectrometry (CIS) experiment for the measurement of the three-dimensional distribution functions of the principal ions in the near Earth space. Such measurements, in combination with electric and magnetic field observations, enable insights in fundamental plasma processes, such as magnetic reconnection and turbulence. More in general, Cluster II is an extraordinarily successful mission which, throughout long lasting and continuous three-dimensional observations across all the key regions of the near-Earth space (e.g., the solar wind, bow shock, geomagnetic tail plasmasphere and auroral acceleration region), led to a variety of new and important discoveries on fundamental processes relevant to the Space Weather discipline (Paschmann et al., 2005; Escoubet et al., 2013, 2015). In addition, joint-analysis of data from Cluster and ground-based radars provided important insights in Space Weather science.
Among others, it has been possible to demonstrate that there is a large amplitude of cusp dynamics even in response to moderate solar wind forcing (Opgenoorth et al., 2001).

Breakthrough results are expected in the future from the study of the data that will be provided by the scientific payload on board the ESA Solar Orbiter mission (to be launched in February 2020). Solar Orbiter has an operational orbit characterized by a perihelion of only 0.28 AU that will allow to observe the surface of the Sun at very high spatial resolution. Furthermore, thanks to its orbital inclination of more than $30^{\circ}$ with respect to the solar equator, it will be possible, towards the end of the mission, to observe for the first time the polar regions of the Sun. The high resolution imaging together with the measurements provided by the in situ instruments, will permit to unveil the mechanisms underlying the generation and heating of the coronal plasma. The Solar Wind Analyser (SWA) is an on board instrument suite of the Solar Orbiter Mission devoted to the study of the composition of the solar wind (UK PI-ship). In situ measurements are indeed necessary for establishing the fundamental physical links between the Sun's highly dynamic magnetized atmosphere and the solar wind in both its quiet and disturbed states. SWA is composed by four units: two Electron Analysers Systems (EAS1 and EAS2), one Proton Alpha Sensor (PAS) and one Heavy Ion Sensor (HIS), developed by the UK. All these sensors are connected to a Data Processing Unit (DPU) developed in Italy with the fundamental support of the industry (TSD; Sitael; Leonardo; Planetek), under the scientific responsibility of INAF-IAPS (Co-PI-ship). The DPU is in charge of supporting the overall instrument functionalities related to power, control, temporary storage, communication and computational capability.

\subsubsection{Space-based instrumentation for Planetary Space Weather measurements}

In the context of Planetary Space Weather, the Italian scientific community leads two important experiments which already provide (in case of JUNO/JIRAM) or will provide (in case of BepiColombo/SERENA) new insights in space-environment interactions in Solar System regions other than the Earth's.

The Search for Exospheric Refilling and Emitted Natural Abundances Experiment (SERENA) on board the ESA/JAXA BepiColombo mission to Mercury, is under INAF-IAPS leadership (Orsini et al., 2010). SERENA is a suite composed of four units of complementary neutral and ionized particle detectors that can provide information on the whole surface-exospheremagnetosphere coupled system of Mercury and on the processes therein; such processes depend strongly on the solar activity and their detailed study provides important insights on the evolution of the Space Weather phenomena in the vicinity of Mercury. The Emitted Low-Energy Neutral Atoms (ELENA) detector, part of the SERENA suite, has been developed completely in Italy. ELENA is a neutral particle camera that investigates the neutral particle release from the surface of Mercury, the exosphere dynamics and the related physical mechanisms as well. ELENA measures energetic neutrals between $20 \mathrm{eV}$ and $5 \mathrm{keV}$. Such measurements allow imaging on the planet's surface of those regions where solar wind and/or magnetospheric ions are actually precipitating, inducing particle release 
through sputtering and back-scattering processes. ELENA was developed with the fundamental support of the industry (OHB Italia, AMDL).

The Jovian Infrared Auroral Mapper (JIRAM) is an imaging spectrometer on board the NASA's Juno spacecraft (Adriani et al., 2017), which started its prime mission around Jupiter on August 2016. The JIRAM investigation (leaded by INAF$I A P S$ ) was purposely designed to study the Jovian aurorae in response to plasma precipitation on the planet's upper atmosphere (Mura et al., 2017). Jupiter's IR aurorae is one of the strongest manifestations of planetary Space Weather within the giant planet system. Precipitating electrons ionize the $\mathrm{H}_{2}$ component of Jupiter's upper atmosphere, leading to the formation of excited $\mathrm{H}_{3}{ }^{+}$, which upon de-excitation emits in the infrared spectral range. The JIRAM measurements, therefore, provide important information for understanding the coupling between the magnetosphere and the atmosphere in a giant planetary system. So, in terms of comparative planetology and Space Weather, the JIRAM measurements can be of significant importance also for understanding the processes at our own system. JIRAM was developed with the fundamental support of the national industry (Leonardo).

Last, radar instruments can also provide important information on the variability of planetary ionospheres at a given location and for a given solar activity level. The Mars Advanced Radar for Subsurface and Ionospheric Sounding (MARSIS), financed by both ASI and NASA, on board the ESA Mars Express mission, was developed by the University of Rome "La Sapienza", with the fundamental support of the industry (Thales Alenia Space Italia). This experiment provided, indeed, a series of data which have been further used also in the context of planetary Space Weather studies related to other missions (e.g., Bergeot et al., 2019).

\subsubsection{Add-on science with interdisciplinary payload}

The "Astro-Rivelatore Gamma a Immagini Leggero" (AGILE) is an X-ray and Gamma ray astronomical satellite of the Italian Space Agency. AGILE's instrumentation includes a Gamma Ray Imaging Detector (GRID) sensitive in the $30 \mathrm{MeV}-50 \mathrm{GeV}$ energy range, a SuperAGILE (SA) hard $\mathrm{X}$-ray monitor sensitive in the $18 \mathrm{keV}-60 \mathrm{keV}$ energy range, a Mini-Calorimeter (MCAL) non-imaging gamma-ray scintillation detector sensitive in the $400 \mathrm{keV}-100 \mathrm{MeV}$ energy range, and an Anticoincidence System (AC), sensitive to hard photons and particles. Although the main science objectives of the AGILE mission are not directly related to Space Weather, nevertheless the related data can provide monitoring of the solar activity. In particular, the anticoincidence panels exposed to the Sun are able to detect solar flares (in the $\mathrm{X} / \mathrm{hard}-\mathrm{X}$ ray range) with high efficiency, and perform quick data processing. Possible correlations of the AGILE archive data with those obtained by other assets (e.g., SOHO, PAMELA) could provide new insights in solar energetic particle sources and propagation processes.

In the future, the ESA Laser Interferometer Space Antenna (LISA) mission, featuring the first interferometer for gravitational wave detection in space, will carry particle detectors optimized for proton and helium integral flux measurements above $70 \mathrm{MeV} / \mathrm{n}$ as well as detectors for solar electron detection in the 1-7 MeV energy range. The latter will allow the forecasting of high-energy SEP events. LISA will consist of a triangular constellation of three spacecraft with the centre of mass orbiting on the ecliptic at a distance of 50 million $\mathrm{km}$ from Earth in the L5 Lagrange point direction. The LISA spacecraft will cover about $1^{\circ}$ in longitude and will remain orbiting $20^{\circ}$ in longitude behind the Earth thus constituting the first natural observatory for high-energy SEPs at small and large intervals in longitude. In this context, the role of the Italian participation is to estimate the effects of high-energy particles in charging the test masses and to study the galactic cosmic-ray short-term variations and SEP evolution with time and space aboard the three LISA satellites (UNIVURB, INAF-IAPS). For an example, the reader is referred to Armano et al. (2018).

\section{Key challenges of Space Weather research}

Consistently with current international research efforts, we discuss below some open questions in the field of Space Weather science, providing a vision on how to map the related key challenges into useful knowledge. Given the scope of this paper, we focus our analysis on the key open areas where the Italian expertise can provide an advance and/or major improvement, anticipating that the list of the topics discussed here is not exhaustive. The related recommendations are presented in Section 4. We also discuss some ideas on space instrumentation deployment that can deliver major insights in Space Weather science (see Sect. 4.3).

\section{Sun-heliosphere domain}

The future availability of more accurate and specific Space Weather forecasting services has as necessary requisite the detailed characterization of the Space Weather drivers. To address the related open question "What is our diagnostic capability for the drivers of Space Weather?", we need to take advantage of current observations of the domain near the Sun and of the models for the event propagation from its source to the heliosphere. In this perspective, we need to advance our knowledge on the physics of the solar atmosphere, the evolution of magnetic regions on the Sun, the triggering of transient and eruptive events, in order to possibly enable pre-event forecasts of solar flares, the origin of CMEs, and related phenomena such as SEP, X-Ray, EUV and radio wave emissions. Major challenges in this context would be to predict magnitude of flares and eruptions and to establish what determines whether an event on the Sun results in SEP release in the heliosphere. Currently, numerous Space Weather forecasting techniques are based on coronagraph observations at L1 (e.g., from LASCO on board the ESA/NASA SOHO mission and STEREO) and heliospheric images (e.g., from STEREO), as well as radio emissions both from the Sun and the solar wind (e.g., Bisi et al., 2010). Current research is also focused on the determination of the properties of the coronal magnetic field prior to an eruption, on the basis of photospheric vector-magnetic maps, X-ray and EUV imaging and coronal polarimetric measurements sensitive to field direction (see, for instance, Bak-Steslicka et al., 2013). Systematic coronagraph imaging and high sensitivity high 
altitude coronal EUV imaging, potentially off of the Sun-Earth line, together with a significant advance in coronal-field modeling, are critical for delivering useful insights on Space Weather and to prepare the scientific background for accurate future Space Weather forecasting.

The propagation of ICMEs and SEPS in the heliosphere is the critical follow-on of the initial phase of solar eruptions. Related long-standing open questions are "How do SEPs propagate in the heliosphere? What establishes the characteristics and propagation of ICMEs, including the interaction with the background solar wind?". In this perspective, we need to dedicate effort in advancing our knowledge in basic space physics, to better study the propagation and evolution in the interplanetary space of HSSs, CIRs, and ICMEs. The challenge here is to develop data-driven models for the propagation of CMEs and SEPs in the heliosphere that take into consideration the dynamic state of the interplanetary environment (e.g., through solar wind measurements), using a wide range of solar wind data. In this way, we may be able to perform more reliable and early forecasts for the arrival in geospace of SEPs, interplanetary shock waves, ICMEs and HSSs.

GCRs and, occasionally, relativistic SEPs with energy larger than $\sim 500 \mathrm{MeV} /$ proton, can penetrate the terrestrial atmosphere provoking extended cascades of neutrons, protons, muons, and other secondary particles. Depending on their energy and direction, such relativistic SEPs can be measured on the ground (at those latitudes where the magnetic cut-off rigidity is smaller than the particle rigidity) through the nucleonic component of the respective atmospheric cascade, registered at ground-based neutron monitors (generating a GLE event), providing crucial information on the high-energy tail of the SEP spectrum (see, e.g., Plainaki et al., 2009b, 2014). It is not yet clear why only a limited number of SEP events results in GLE events. One key open question in this context is the following "What are the critical physical conditions determining the extent of the SEP energy spectrum". Current research has been long focused on the modeling of GLE events through the use of extended neutron monitor networks (e.g., NMDB). The challenge here is to better constrain the particle radiation transport within the magnetosphere, through the incorporation of magnetic field and radiation data (e.g., GEO and LEO data), to significantly improve current GLE models towards forecasting and nowcasting opportunities.

\section{Magnetosphere-ionosphere domain}

The most critical part of the Space Weather puzzle comes with the interaction of solar plasma structures with the terrestrial magnetosphere which results in geomagnetic variability. The open question in this context is "What is our capability for predicting the geo-effectiveness of an ICME event?". In this perspective, the continuity of research work aiming at the understanding of the ICME evolution through the bow shock, of the solar wind-magnetosphere-ionosphere-thermosphere coupling and the radiation belts and the plasmasphere dynamics, should be ensured. Basic physics, therefore, can be transformed in useful knowledge for describing fast dynamical processes and disruptive phenomena in the geospace, revealing the details of how energy is transported and stored in the magnetosphere and finally released from the magnetotail to the ionosphere or into the interplanetary space (from the tail). In particular, to obtain a deep understanding of the physics behind the magnetospheric substorms, it is necessary to expand current knowledge of the mechanisms that enable solar wind plasma, momentum and energy transfer into the magnetosphere and to better perceive how reconnection acts in the magnetotail. Important advances in this area have been obtained after the THEMIS and Cluster missions, as well as by the NASA/MMS mission, but many compelling questions still remain open. Coordinated ground-based and multipoint, multi-scale in situ observations are needed to address them properly. The knowledge obtained thanks to such observations should be embedded in the best way possible within the models of the magnetosphereionosphere coupling. In this framework, another important challenge to better understand the mechanisms of the storm/ substorm development is the continuous mapping of the ring current population.

Another key open question in the area of magnetosphereionosphere coupling considers the generation of GICs after the occurrence of large rate of change of the surface magnetic field: "What are the key physical parameters allowing the prediction of GICs?". While the detailed properties of GICs depend on the structure and interactions of the inducing currents, ground conductivity, and network components, a necessary condition for any potentially damaging GIC is the occurrence of a large rate of change of the horizontal component of the magnetic field on the ground. This means that it is fundamental for the GICs prediction to understand the physical sources of these large magnetic field perturbations which are generally due to the sudden variations of external electric current systems such as magnetopause, ring current, equatorial electrojet and auroral ionospheric electric currents that generally increase during geomagnetic storms and substorms. As stated also in the past (see Schrijver et al., 2015), the link between physical processes (e.g., energy transport, dissipation) and the way energy is released (e.g., explosive or gradual) is not yet clear. The challenge here is to obtain a comprehensive view of the evolution of the entire magnetosphere-ionospherethermosphere system. The key in this research is to identify the pre-existing (to the GIC) conditions of the system (e.g., possible heating of the underlying atmosphere, the ring and tail current state, etc.) which determine the way stored energy is dissipated. Systematic studies on ground-conductivity, especially in the regions where the probability of GIC appearance is higher, is a priority.

The forecasting of ionospheric perturbations requires a deeper understanding of the physical parameters determining the chemical composition of the atmosphere, its density, temperature and dynamics, the atmosphere-ionosphere coupling, and, on rare occasions (i.e. when the energy cut-off of $\sim 500 \mathrm{MeV} /$ proton is overcome), the solar energetic particle precipitation in the upper atmosphere. The variability of the electron density in the ionosphere depends on physical mechanisms that are coupled with solar activity, magnetospheric processes, and the mechanisms taking place within the thermosphere. The challenge here is to better integrate current physical models by considering the coupling of these subsystems. Data assimilation from both space-based instruments and groundbased networks can benefit such efforts at large.

In the magnetosphere-ionosphere domain, the overall challenge is to develop global Space Weather monitoring techniques and algorithms, based on advance scientific models which 
encompass all the physical processes that affect the morphology of the ionosphere (including its coupling with the thermosphere, plasmasphere and magnetosphere) and on the exploitation of currently available datasets. In parallel, it is very important to develop reliable Space Weather alerts for geospace disturbances, geomagnetic storms, substorms, and GICs, and techniques for monitoring the related variability of the ionosphere and atmosphere. To this end, space and ground-based data are required from all parts of the coupled system (i.e., the atmosphere, the ionosphere, the inner magnetosphere, the magnetotail, and the lobes). Such information will contribute to the achievement of a multi-point characterization of the entire system. Data calibration is an important task to correctly incorporate different types of data in a global model.

\section{Technological and biological Space Weather impacts domain}

Forecasting of the Space Weather impacts relies, certainly, on our ability to predict the Space Weather phenomena and hence on the advances described in the two previous sections. For this reason, support of current research activities in the solar, heliospheric, magnetospheric and ionospheric domains remains a top priority. The crucial open questions in the area of Space Weather impacts, aligned with the pathways provided in the COSPAR and ILWS roadmap, are "What is our capability to provide an accurate estimation of the near-real time conditions of the particle environment of space assets? What is our capability to obtain accurate forecasts of the ionospheric variability due to Space Weather events? What is our capability for the accurate prediction of extreme Space Weather events?". Indeed, one of the key Space Weather research challenges today is the need to better assess the uncertainties in our ability to forecast future Space Weather conditions. From one side, an advance in our understanding of the related physics can definitely bring some improvement. On the other hand, there is an intrinsic uncertainty in Space Weather phenomena which enforces a need for statistical forecasts. A cross-cutting example is the triggering of magnetic reconnection, a process that exhibits a self-organized criticality. Another major source of uncertainties is the limited number of observations driving forecast models; this fact, indeed, forces the use of ensemble methods to limit uncertainties. Addressing of the above issues, also on the basis of physicsbased modeling, is a long-term requirement for the development of efficient tools for the prediction of Space Weather technological and biological impacts. Moreover, the lessons learned from work in Space Weather operations and services can be a powerful stimulus to science, allowing the development of new ideas, originating from Space Weather risk assessments and mitigation action plans. These $\mathrm{O} 2 \mathrm{R}$ activities, therefore, can be an important aspect of the overall plan of the scientific work related to Space Weather, such as the Research to Operations (R2O) pathway is crucial for Space Weather mitigation. In this context, a continuous iteration between the scientific community, end-users and service providers, is an efficient pathway toward a global progress in the field of Space Weather.

The functionality of communication, satellites positioning, and navigation systems depends significantly on the ionospheric variability hence systematic targeted research activities aiming also at the development of accurate forecasting and now-casting techniques, are a basic requirement for the overall progress in the field of Space Weather. The availability of accurate ionospheric plasma measurements with sufficient spatial coverage is a challenging point within upcoming research activities, in particular in view of physics-based ionospheric modeling, together with data assimilation. What is a science challenge is the joint-analysis of interdisciplinary data (e.g., magnetometer, ionosondes, and GNSS data), to feedback models of transionospheric radio wave propagation.

Current research has been focused on the analysis of the Space Weather biological and technological impacts, also in view of human space exploration, however, the lack of systematic space-based measurements results in difficulties in the accurate prediction of events that provoke spacecraft failures or can be a risk for the astronaut health. To advance current forecasting techniques for Space Weather impacts on technology, as a first step, we need to obtain a deep understanding of the radiation environment and of its variability. In this context, we need first to advance our understanding of the background GCR modulation by solar activity. In parallel, to enrich our knowledge on long-term effects, such as TID, displacement damage or chronic exposure to GCR and SEP radiation, in view of both robotic and human space exploration. Such a research activity can benefit largely by joint engineering and impact studies of the dependence of the so far registered failures (of components and systems on board spacecraft) on the radiation environment conditions. Further understanding of short-term or transient effects (e.g., single event effects, SEE) of radiation in avionics and electronic systems on board spacecraft and on the ground, is also necessary. Systematic study of the links between precursors and the core of the SEP event dynamics could result in a major advance in the area of Space Weather forecasting and now-casting (Ippolito et al., 2005). Based on the above, a technological challenge is the development of optimized instruments to measure in situ the radiation environment, providing, e.g., contemporary coverage of more than one regions of interest. The long-term challenge is to achieve the best combination of forecasting and now-casting techniques to better address Space Weather mitigation (e.g., fast forecasting and without false negatives), also in the context of deep space human exploration, taking into consideration the past and current conditions of the radiation environment at the point of interest.

\section{Planetary Space Weather domain}

With increasing efforts in space exploration, the need for an in depth understanding of the space environments around planetary bodies other than Earth emerges. Interdisciplinary work in the field of Planetary Space Weather is required to provide environmental specification for the design and maintenance of spacecraft and systems in space. Of key importance is the improvement of our ability to predict the fluxes of energetic particles that can be detected when a shock passes by a spacecraft. The key open question in this context links to the ones aforementioned: "What is our capability for predicting the environment conditions at different locations in the Solar System given the current space assets?'. Charged particles can pose major Space Weather hazards (see Koskinen et al., 2017 and references therein). Since, the related events are due to the arrival (at the location of the spacecraft) of a shock, the detection of type II radio-burst can function as a good proxy. 
Another aspect of major importance is the study of Space Weather phenomena in a comparative planetology framework, meaning to investigate the physical processes of space-environment interactions in the vicinity (or within the system itself) of different planets in the Solar System. Such an approach will help to understand the physical phenomena at large, pushing current models to their limits. A challenging example of this idea is to comparatively study the auroral processes at Jupiter and Earth, two strongly magnetized planets of our Solar System. In general, the advances described in the two previous sections can certainly benefit the work in the field of Planetary Space Weather, hence support of current research activities in all the circumterrestrial Space Weather domains, is of particular importance. In this perspective, it is necessary to determine the properties of the solar wind and the characteristics of its interaction with the planetary environments at different distances from the Sun, potentially out of the ecliptic plane also, to obtain a global and complete framework of Space Weather within the Solar System. To map these goals to useful knowledge, it is necessary also to consider potentially extreme conditions of Space Weather to take into consideration in the models. The challenge here is to achieve a multi-year environment variability model driven by the solar activity patterns. Synergies between solar, plasma, planetary and stellar communities can improve our ability to predict extreme Space Weather in the Solar System.

\section{Roadmap's detailed recommendations}

To convert the challenges presented in Section 3 into actions and methodologies to be implemented within a logical timeframe, it is necessary to define recommendations for coordinated and consolidated activities, that meet the different needs of the Space Weather community. A schematic view of the proposed Space Weather roadmap interconnecting Research, Observations, Payload development, and potentially future Space Weather services, is reported in Figure 5. The common goal of the proposed recommendations is to obtain a deep understanding of Space Weather science to set the basis for future forecasting applications. This includes instrumentation, data analysis, modeling, and theoretical research. Below, we group the proposed recommendations into six main groups: observational and theoretical research recommendations (Sect. 4.1); maintenance of existing facilities (Sect. 4.2); study of space mission concepts and deployment of new instrumentation (Sect. 4.3); development of a national scientific Space Weather data centre (Sect. 4.4); teaming and collaboration between ASI and the scientific community (Sect. 4.5); education, training, and public outreach (Sect. 4.6). The whole set of recommendations has been defined on the basis of the following requirements:

- observational coverage of Space Weather parameters;

- data archiving, sharing and data standardization - as far as possible - among different research communities;

- data-driven and ab-initio theoretical models to be used for the analysis of Space Weather events in view of future now-casting/forecasting services;
- interaction among interdisciplinary research communities at national and international level, users and agencies;

- academic education and outreach actions.

\subsection{Observational and theoretical research recommendations}

From a scientific research point of view, aligned at large extent with recent international studies and planning actions (e. g., the COSPAR and ILWS roadmap for Space Weather; Schrijver et al., 2015), current observational and theoretical needs in the field of Space Weather science may be distinguished in three main directions: I. Scientific data-driven modeling; II. Basic physics behind Space Weather; and III. Space Weather now-casting, forecasting and impact analysis. It is underlined that all three groups are conceptually and effectively linked so that the respective recommendations are often interconnected. We provide below our view of what are the areas with potential novel science in the field of Space Weather, and discuss recommendations and approach concepts for prioritizing them, based on the strengths of the Italian scientific community. Moreover, we attempt to expand these recommendations in an international context with the scope to point out how international collaborations are expected to enhance the value of Space Weather science in Italy. We emphasize that that the proposed recommendations and approach concepts may well be modified in the next years on the basis of possible new top level science needs (defined at national or international level) hence the suggested pathways constitute only options.

\section{Direction I: Scientific data-driven modeling}

Need: To advance current Space Weather models on the basis of new innovative approaches including interdisciplinary data fusion and ensemble modeling where possible.

Recommendations: Preference should be given to the development of data-driven models, guided where possible by the observations of the evolving conditions in the entire modeled volume. Very often, Space Weather models are based on snapshot observables and/or extrapolations, limiting our global understanding of the coupled mechanisms taking place from the Sun all the way down to the Earth. We recommend direct or indirect guidance of all modeling efforts in the Space Weather-related science fields presented in Section 1 by observational data, preferably obtained through different assets (ground- and/or space-based). The focus should be on the development of models as research tools, which will allow, at a second step, transition to forecasting techniques. Such an approach has been already adopted by the COSPAR and ILWS committees (Schrijver et al., 2015) and it can result in a major advance in our current understanding of Space Weather. In this context, the exploitation of the available archive data is a major priority. Data assimilation should support the development of Space Weather models in a comprehensive way. To efficiently facilitate such efforts, online access and tools to transform data sets into standard formats should be provided (see also Sect. 4.4). The continuation of the existing observation and computational capabilities necessary for data modeling for 


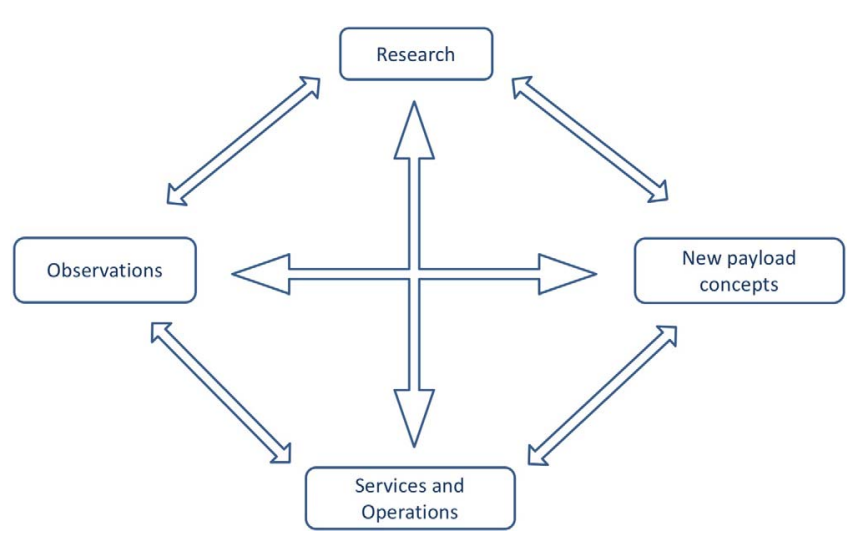

Fig. 5. The conceptual structure of Italy's roadmap towards Space Weather science. The proposed roadmap brings together all key constituents of the national Space Weather system, facilitating their interconnection through recommendations that point towards the establishment of targeted partnerships and scientific synergies. The establishment of a scientific Space Weather data center, in ASI's $S S D C$, as a prototype structure hosting data archives and tools and/or offering a centralized access to Space Weather related resources, could have a major role in view of future application and service development in Italy. The whole structure is intended in the frame of a collaborative environment for scientific research and technology activities.

scientific purposes is a major priority. Indeed, the currently available assets observing the heliosphere offer the best possibility for concrete scientific modeling towards a better understanding of Space Weather at global scale.

\section{Possible approach concepts:}

- to develop data-driven models of the magnetic field of the solar active regions to assess energetics during CME and flare events;

- to investigate magnetic fields and energetics in CME source regions associated with quiescent eruptions;

- to develop improved models for differential response functions of ground-based neutron monitors through the comparison of ground observed rates with simultaneous charged particle fluxes measurements in LEO to fully exploit the potential of historical and current data sets from ground-based monitoring facilities with the scope to characterize the SEP and GCR time dependence;

- to develop improved SEP-GLE models through the incorporation in the codes of magnetic field and radiation data (e.g., from LEO).

Within an international context: To constrain models of the magnetic field above the active regions and in regions associated with quiescent eruptions, 3-D information of the coronal loop geometry is of particular importance. This could be achieved by spacecraft observations of the Sun-Earth line, combined also with data (e.g., EUV images) obtained by existing instruments onboard international missions (e.g., SDO, Hinode). Considering the investigation of the global photospheric magnetic field, synoptic maps (obtained from ground and spacebased observatories) are often used; such maps, often contain interpolated data that correspond to regions where the field is poorly observed (e.g., poles). Also in this case, photospheric magnetograms off of the Sun-Earth line could complement the existing information. ESA's Solar Orbiter mission is expected to provide information for the calibration of the high latitude magnetic field, although only occasionally. Moreover, to accurately couple space-based SEP and GCR flux data in the near-Earth space with ground-based fluxes, multi-point in situ and ground based observations (e.g., from GOES, ACE, and the NMDB network, respectively) are necessary.

\section{Direction II: Basic physics behind Space Weather}

Need: To understand Space Weather origins at the Sun and their propagation in the heliosphere to further advance current modeling.

Recommendations: Advanced modeling of the coupling of a Space Weather source region to a specific point in the heliosphere, requires knowledge of basic physics, the existence of a wide range of observational parameters, and also forward modeling capabilities (in addition to the data-driven modeling capabilities for Direction I) for the dynamic magnetic field, solar wind plasma and energetic particle populations. We recommend hence the continuation of theoretical studies related to Space Weather in parallel with a continuous and systematic exchange of feedback between new observational data and theoretical estimations. Attention should be given in the continuation of existing computational capabilities, necessary for an in depth study of the physical phenomena related to Space Weather. As stated also in the COSPAR and ILWS roadmap, to understand the coupling of the solar wind to the magnetosphere and ionosphere and strong GICs, new space-based multi-point data for the contemporary study of the plasma in the inner edge of the plasma sheet and in the near-Earth domain of the magnetosphere is needed (link to Direction I). Collaboration within Italy and among international teams working on similar topics is very important.

\section{Possible approach concepts:}

- to advance our insights in and understanding of the mechanisms of energy transfer and release to the solar atmosphere during flare events, through the improvement of the existing numerical MHD models;

- to investigate the mechanisms that determine the heating of the solar wind plasma and the acceleration of SEPs as well as to stimulate the further study of the solar wind streams, CIRs, CME-driven shocks and SEP propagation in the inner heliosphere;

- to study the aerodynamic drag experienced by a typical ICME due to its interaction with the ambient solar wind; determine the drag coefficient for different solar wind conditions and initial CME parameters;

- to improve the understanding of plasma processes enabling the mass and energy transfer from the solar wind into the magnetosphere, across the terrestrial magnetopause, to quantitatively assess the geo-effectiveness of specific interplanetary disturbances;

- to study the cold plasma distribution in the inner magnetosphere and its interaction with the more energetic particle populations of the ring current and the radiation belts; 
- to better understand the spatial configuration of the reconnection region within the magnetotail and its dynamics; to investigate the physics of the plasma jets and their interaction with the near Earth's magnetospheric region; to advance our understanding in the large-scale changes of the magnetotail configuration and the distributions of the charged particle populations within; to improve ring current modeling; to advance our insights in the storm/ substorm development;

- to study the geomagnetic activity in the ULF range and its influence on the precipitation of energetic electrons into the high latitude atmosphere and investigate the corresponding atmospheric response;

- to study the dynamics and coupling of magnetosphereionosphere-thermosphere system and its response to solar wind and magnetospheric forcing taking into account that many of the natural coupling processes within this system are linked through complexity processes including feedback, nonlinearity, instability, preconditioning, and emergence behavior;

- to advance our understanding of the GCR propagation in the heliosphere and its modulation by solar activity;

- to advance our insights in and understanding of the solar and magnetospheric plasma interactions with different planetary environments in our Solar System to obtain a global view of Space Weather and to interpret the related mechanisms through a common scientific frame.

Within an international context: Current research challenges in the field of Space Weather science often require a globally coordinated approach. For instance, considering the heliosphere domain, the study of large-scale variability in the terrestrial magnetotail and the distributions of the embedded particle populations can benefit largely from the joint-analysis of data of different type, obtained from different past and ongoing missions (e.g., SuperDARN, SuperMAG, Cluster, THEMIS, and MMS). Equivalently, to effectively understand the evolution of SEP events (e.g., peak intensity, duration) and how it depends on the source regions, the joint-study of the properties of the near-Sun interplanetary space and geospace is necessary; in particular type II and IV radio burst data should be analyzed together with multi-point in situ observations of particle and fields (e.g., from GOES, ACE). Moreover, the development of integrated models for the entire inner heliosphere can be supported by targeted collaborations which will increase the value of Space Weather research in Italy.

\section{Direction III: Space Weather now-casting, forecasting, and impacts analysis}

Needs: To contribute to the improvement of the space environment specification to ensure astronaut safety and to allow design criteria for technological space components and infrastructures to resist and/or recover from Space Weather impacts; to develop ionosphere Space Weather forecasts, guided by physics-based models, in relation to current monitoring capabilities, to ensure the proper function of telecommunication and navigation systems; to develop radiation forecasting models that will allow the design of mitigation techniques to ensure airplane passengers health during extreme Space Weather events, and to avoid possible on board system failures.
Recommendations: In view of Space Weather now-casting and forecasting, we recommend the best possible coordination of current ground-based and space-based networks of instrumentation and the development of test-beds to speculate on the retrieval of key parameters of the space environment variability. We also recommend the development of particle environment now-casting and forecasting models for LEO and GEO based on multi-point observations of both particle and fields, and taking into consideration the lessons learned from scientific modeling (see Direction I). The focus here is on the interdisciplinary approach in observation-based modeling of the radiation environment (link with Direction I). In the context of both robotic and human space exploration, the establishment of the conditions to be encountered by the spacecraft is a necessary requisite that will allow the development of payload instruments capable of surviving Space Weather events. Aligned to the COSPAR and ILWS roadmap, we recommend the definition of specifications for all relevant Space Weather phenomena (i.e., SEP events, solar irradiance variability, geomagnetic variability, etc.) that may be encountered wherever human technologies are deployed. Focus should have given on the definition of extreme limits in solar, heliospheric and geomagnetic conditions (i.e. case of extreme Space Weather events). In the context of such a modeling approach, inter-calibration of datasets from space and ground, maintenance of existent Space Weather assets and additional instrumentation to complement current capabilities, are priorities. We note that it would be a major advance if now-casting modeling covered, as much as possible, the entire inner heliosphere to allow better validation of the techniques applied to the circumterrestrial Space Weather case and to further prepare for human and robotic exploration of the Solar System. An in depth analysis of the failures during historical Space Weather events will be essential for the design of new nowcasting and forecasting algorithms (link with Direction II).

The ionospheric variability due to Space Weather has some important effects on both communications and satellites positioning and navigation systems. Indeed, precise positioning and navigation, as required in aviation, can be at risk due to temporal variability of the ionosphere plasma density. Radio scintillations caused by plasma turbulence can result, in severe cases, in the complete loss of the positioning and navigation signal. To observe and, finally, predict, ionospheric variability driven by Space Weather, a systematic approach also in the related research fields, aiming at the development of accurate forecasting and now-casting techniques, is required. Indeed, there is an urgent need to have alerting systems for potential disruptions of GNSS signals, airplane HF and satellite communication disturbances, and radio wave absorption in the frequencies from VHF to S-band. Physics-based modeling together with data assimilation (from both space-based instruments and ground-based networks) is a priority (link with Direction I). Analyses based on magnetometer, ionosondes, and GNSS data can be complemented by solar spectral irradiance measurements, to advance our overall understanding of the ionospheric processes influencing trans-ionospheric radio wave propagation. In particular, electron density data, coming from the Italian network of ionosondes, can play an important role in the investigations of ionospheric phenomena, such as the sporadic-E layer disturbances, which strongly affect radio communications in the HF range and low VHF range (Rice et al., 2011). 
As stated also within the COSPAR and ILWS roadmap, although data-assimilation has been used within some specific research projects, a comprehensive approach indicating how such assimilation can help ionospheric models, is still missing. We recommend the support of scientific research in this field, with special attention in the validation of the related ionosphere Space Weather models and forecasting codes at the entire range of latitudes (from low to high geographical latitudes). The ground-based networks providing the related data (e.g., magnetometers, ionosondes, GNSS receivers) should be maintained. Data dissemination is strongly recommended.

In the end, aligned to the NASA LWS Institute GIC Working Group (GIC science, engineering and applications readiness; Pulkkinen et al., 2017), the impact of Space Weather on long engineered conductor systems such as power grids, pipelines and railway systems must be studied and GIC mitigation actions must be proposed. In this framework, the impact of Space Weather on power grids in Mediterranean countries, such as Italy, needs deeper assessment, including consideration of coastal effects, ground conductivity, and failure reports. The knowledge of the ground conductivity structure, which is responsible for the way the electromagnetic field and GIC on the surface of the Earth respond to external magnetosphericionospheric electric current variations, is probably the most challenging point un this investigation. The poor knowledge of the local ground conductivity is indeed the dominant source for GIC modeling uncertainty. It is thus of major interest from the GIC standpoint to extend the electromagnetic sounding campaigns to cover all key areas of GIC interest. At the same time, it is also important to be sure to have a good spatial coverage of geophysical observatories that allows space weather applications taking into account that the fluctuations in the geomagnetic signature that drive the geoelectric field can be highly complex and localized (e.g., Ngwira et al., 2015; Pulkkinen et al., 2015).

\section{Possible approach concepts:}

- to observe the inner heliosphere at radio wavelengths to study shocks and electron beams during Space Weather events;

- to develop long-term forecasts (from hours to days) for the arrival of ICMEs in geospace, based on an in-depth understanding of both the Space Weather origin at the Sun and the propagation of magnetic structures in the heliosphere (link to Direction I); apply drag-based models to simulate the ICME propagation in the interplanetary space to predict the arrival time at Earth;

- to develop reliable and fast forecasts of SEP occurrence and evolution in the near-Earth space environment;

- to develop improved alerts for geomagnetic disturbances and GICs on the basis of an advanced understanding of the factors that control their generation;

- to improve the existing now-casting of the geomagnetic and ionospheric variability using the existing geomagnetic observatories and ionospheric stations and HF radars;

- to improve theoretical and (semi-)empirical ionospheric modeling, consolidate benchmarks for its validation and improve the current capability of translating ionospheric models into algorithms able to forecast the spatial and temporal ionospheric variability with the longest forecasting horizon;
- to develop predictive data-driven models for mid/ long-term forecasts of the GCR radiation level to be used in the assessment of radiation risk during interplanetary space missions;

- to potentially develop global assimilative integrated models of the Earth's radiation environment towards forecast development, including the magnetosphere, ionosphere, thermosphere, and atmosphere to provide an efficient tool for the monitoring of the harsh radiation in geospace; to validate these models based on archival information (link to Direction I);

- to develop radiation forecasting models for the entire inner heliosphere region to obtain a global picture of the space environment variability to better prepare future human and robotic Solar System exploration missions (link to Direction II).

Within an international context: Quantitative and realistic assessment of the prediction of the variability of the space environment as a function of specific input parameters, will be greatly enhanced through an international collaborative approach. The estimation of the geo-effectiveness of an event, based on both the environment monitoring and the available scientific models, is possible through the contemporary use of different international Space Weather assets which provide sufficient spatial and temporal coverage. A multi-point observation approach, therefore, possible through the use of data from different missions is the key for the comprehensive monitoring and prediction of the variability of the space environment at different locations in the Solar System. Furthermore, the use of both ionosonde data from the international network and GNSS data, offers the possibility for a multi-parametric approach in the investigation of Space Weather related ionospheric phenomena, such as the traveling ionospheric disturbances.

\subsection{Maintenance of existing facilities}

Monitoring facilities constitute the core capacity for any activity related to Space Weather science, with particular focus on data-analysis or data-driven model development. As stated within the COSPAR roadmap (Schrijver et al., 2015), coordination between the various space- and ground-based solar observatories in observing campaigns of particularly active space-weather source regions on the solar surface offer an opportunity for a global "laboratory" of extensive coverage of regions of interest. In addition, both space- and ground-based data are required from all parts of the magnetosphere-ionosphere coupled system, both in (and below) the ionosphere, the inner magnetosphere, the magnetotail, and the lobe region. As discussed also in Section 2.2.1, in Italy a large amount of Space Weather data is obtained through observations made by the national ground-based assets (for a concise summary of the characteristics of these assets, the reader is referred to Tables 2-7), necessary for the study of the Sun, the Earth's magnetic field, magnetosphere and upper atmosphere (plasmasphere included), the GCR background and the related Space Weather phenomena, including impacts on technology and human health. We summarize below current needs and general recommendations considering the sustainability of the Italian Space Weather assets. 
Need: There is a clear rationale of the scientific and practical drivers for the continued operation of the Italian ground-based Space Weather assets. The main points are summarized below:

- integrated measurements, derived from the combination of space- and ground-based data, give in general a more reliable estimate of Space Weather parameters especially due to the lack of a dense coverage in space (e.g., retrieval of the SEP spatial distribution and spectrum; reconstruction of the development of strong GICs, modeling of the dynamic radiation-belt populations; retrieval of the plasma bubble properties and aurora instabilities, etc.);

- calibrated ground-based measurements are often needed to support the retrieval of Space Weather parameters obtained from space-based measurements (e.g., particle and field measurements, etc.);

- systematic ground-based observations are needed for monitoring the upper atmosphere variability, and for evaluating the validity of Space Weather products based either on space-based measurements or on model runs;

- the concept of a multi-point observation and multimessenger approach in Space Weather science is significantly supported by the systematic analysis of both space and ground-based interdisciplinary data.

Recommendations: Taking into consideration the existing ground-based observation facilities in Italy (see Sect. 2.2.1), the following recommendations have to be issued for the Space Weather assets sustainability:

- ground-based facilities for Sun observations and diagnosing of particle flare-acceleration and of CME-related shock waves;

- ground-based sensors for heliospheric, magnetospheric, ionospheric, and geomagnetic data to complement satellite data;

- ground-based neutron monitors for registering the arrival of relativistic SEPs during major Space Weather events and for measuring the GCR background.

\subsection{Study of space mission concepts and deployment of new instrumentation}

General concept or why we need space measurements: Solar activity is the principal source of circumterrestrial Space Weather. The Sun's photon radiation in the UV, EUV and X-ray spectral ranges can be directly monitored twenty four hours a day only from space. The only exception is represented by the possibility to install a coronagraph in the Antarctic region, providing up to three months with the Sun's elevation of $15^{\circ}$ with limited atmospheric turbulences (hence good seeing). The observations of the solar corona with ground-based coronagraphs are limited not only to the clear sky day-time, but also to the inner fraction of the corona (i.e. between 1.0 and 1.2 solar radii), because of noise due to the sky brightness, where halo-CMEs (i.e., those directed towards the Earth) cannot be observed. A much larger coronagraph field of view (i.e., up to 30 solar radii), so as to obtain the necessary images for determining the CME initial speed and direction, is needed.
Even the detection of type-II radio bursts (unambiguous signatures of interplanetary shock-waves) is limited from the ground, because of ionospheric cut-off at frequencies smaller than $\sim 14 \mathrm{MHz}$, corresponding to shock heliocentric distances larger than $\sim 2$ solar radii. The importance of corona imaging from space is further supported by the fact that the proposed future space missions dedicated to the study of the Sun and Space Weather have in their planned payload EUV disk imagers and/or coronagraphs. This is true for instance for the future Chinese ASO-S mission, the Indian Adytia-L1 mission, and the actual efforts made by ESA in collaboration with US partners (NOAA) to develop a future L1/L5 Space-Weather Mission based on a couple of spacecraft located in L1 and L5 Sun-Earth Lagrangian points. Moreover, the Earth's atmosphere is a major obstacle for the relatively low-energy SEPs which do not give ground-signatures for Space Weather events except rare occasions when the SEP energy overcomes the atmospheric/magnetic field threshold generating a GLE event (e.g., Plainaki et al., 2005, 2007). However, even low-energy SEPs can be hazardous for space instrumentation and astronauts therefore the study of their characteristics and properties (e.g., Laurenza et al., 2019), through space-based observations and subsequent data analysis, is of crucial importance for Space Weather. For this reason, particle flux data from space-borne detectors are of crucial importance for Space Weather.

The research in the different Space Weather disciplines can benefit from both new space missions and/or new innovative instrumentation, including also the possibility of rideshare or hosted payload flight opportunities. Thanks to their relatively low cost, reduced sizes and volumes, CubeSats may be a captivating alternative to classic satellites, able to achieve a series of science objectives related to Space Weather. For example, traveling outside the Earth's Van Allen belts, a CubeSats system gives the opportunity to further investigate the space radiation environment (e.g., Viscio et al., 2014). Moreover, Space Weather CubeSats missions may function also as technology validation opportunities, allowing to test advanced technologies (e.g., solar sails) but also the response of miniaturized instruments to harsh space environment, even in view of future human space exploration missions. Moreover, constellations of CubeSats can provide multi-point measurements to support further investigation of the relationships between solar energetic particles, flares and coronal mass ejections, and characterizations of the drivers of ionosphere perturbations related to Space Weather (e.g., Mannucci et al., 2010).

In general, considering new space missions, it would be of major importance for the Italian scientific community to contribute to the international studies of new space mission concepts for monitoring Space Weather, also through the allocation of sensors also away from the Sun-Earth line, and/or at different distances from the Sun. Considering new innovative instrumentation, and based on current national expertise, we provide here recommendations in the following directions. We underline that such directions may well be modified and/or integrated in the next years on the basis of possible new top level needs (decided at national or international level) hence the suggested pathways are only potential options:

1. Solar corona and heliospheric imaging (well off of the Sun-Earth line) to investigate CMEs and solar wind; 
2. ENA imaging for investigating the geomagnetic activity, the planetary Space Weather conditions, and the energetic particle sources on the Sun;

3. VIS-NIR polarimetry for chromospheric and photospheric diagnostics;

4. X-ray polarimetric imaging to reveal the nature of the polarization of hard X-ray sources in the solar atmosphere;

5. Particle detectors to measure the radiation environment in or outside the Earth's magnetosphere;

6. In situ measurements of plasma properties;

7. Space Weather studies with stratospheric balloons.

Considering the aforementioned point (7) we note that it does not actually refer to a space mission concept in a straightforward way. However, as demonstrated several times in the past, space instrumentation is often tested through stratospheric balloon experiments hence important feedback to be take into account during payload development is obtained from such efforts. For this reason, this research and development activity field is mentioned in the current paper. Below we describe briefly some mission/payload scenarios and experiment designs in which the Italian scientific community has a major expertise.

\subsubsection{Solar corona imaging to investigate Coronal Mass Ejections and the solar wind}

The inner solar corona (at heliocentric distances between 1 and $\sim 10$ solar radii) is the region of the Sun where solar flares occur, erupting prominences and CMEs undergo their main acceleration phase, interplanetary shocks are formed and most of the energetic SEP populations are accelerated. This is also the region where coronal heating occurs and where the solar wind is mostly accelerated. This very important region can be directly investigated only through the analysis of remote sensing observations acquired in the EUV, VL, and radio wavelength bands. Even the very challenging NASA Parker Solar Probe mission (launched on 11th August 2018) will never explore with in situ instruments this inner region, because of tremendous energy deposited on the spacecraft thermal shield.

Typically, the solar corona is observed with two different kinds of instruments: full-disk imagers (in EUV) observing the lower corona $(<1.2$ solar radii) in the EUV range, and coronagraphs observing the intermediate and outer corona (between $\sim 1.5$ and $\sim 30$ solar radii) in the VL range. The technique of coronal imaging can be used to provide early Space Weather alerts. The instruments detecting at very first time the occurrence of a solar flare, of a prominence eruption and of a CME are those providing imaging of the solar corona and chromosphere. Whereas ground-based instruments provide valuable information on the distribution of photospheric magnetic fields in the eruption source region and on the occurrence of flares and prominence/ filament eruptions in the hemisphere visible from the Earth (for instance using $\mathrm{H} \alpha$ filters), their observations are limited to clear sky day-time providing no information on possible associated CMEs. During their early propagation phase in the lower corona, solar eruptions undergo significant latitudinal and longitudinal deflections, distortions, accelerations and decelerations, and even rotations, due to the action of the surrounding coronal magnetic fields. All these effects play a key role in the final CME geo-effectiveness. The CME kinematical properties (e.g., speed, acceleration, and propagation direction with respect to the
Sun-Earth line) can be determined in detail only from space. Such information is crucial to forecast the arrival times of CMEs and their possible impact on Earth and can be obtained with space-based full-disk EUV imagers and VL coronagraphs. Moreover, these instruments can also provide important information on the ambient solar wind that will be met by the eruption during its Sun-to-Earth propagation (from observed coronal streamers and coronal holes), hence on the possible occurrence of CME acceleration or deceleration during its interplanetary propagation. For these reasons, coronal imaging from space is of paramount importance for both Space Weather science and applications.

The Italian scientific community has a long-term wellestablished expertise not only in instrument development, but also on corona imaging data analysis and interpretation, thanks to the development of many new diagnostic techniques to determine physical properties of solar wind and CMEs plasmas. For all the above reasons, Italy has now a leading role at European level in particular for space-based coronagraphy.

\subsubsection{VIS-NIR polarimetry for photospheric and chromospheric diagnostics}

The observation of the Sun's chromosphere is crucial for addressing flare activity, an important driver of Space Weather in the inner Solar System. The best diagnostics for photospheric and chromospheric polarimetry lie in the visible and near infrared range of the solar spectrum. Being both of them sensitive to the Hanle and Zeeman effects, they are unique diagnostic tools for solar magnetic fields covering a wide range of strengths. Although the underlying physical mechanisms are complex, spectral line inversion codes to analyze the emergent spectral line radiation in a variety of physical scenarios do exist and are becoming available to the scientific community (Asensio Ramos et al., 2008; Viticchié et al., 2011; Romano et al., 2014). In particular, observations and theory show that the He I triplet (around $1083 \mathrm{~nm}$ ) is most suited for active regions studies, while the CaII line (at $854.2 \mathrm{~nm}$ ) is better employed for quieter conditions. Finally, photospheric, magnetically sensitive lines are available in the immediate spectral surroundings of these two important chromospheric lines: this is crucial in order to obtain quasi-simultaneous maps of photospheric magnetic fields that will aid the interpretation of the polarimetric data in the chromosphere and the extrapolation to higher atmospheric levels. In the context of Space Weather, such an information is of significant importance since it will shed light in the origins of Space Weather on the Sun, providing constraints for the theoretical models of energy transfer inside the solar atmosphere. At a second step, such an information can be particularly helpful in the context of flare forecasting and now-casting, and also during revisions of past-models of solar activity.

In general, spectropolarimetry is one of the most powerful tools to study the solar atmosphere and has the potential to provide new insight into the sources of Space Weather. In the past years, many efforts have been devoted to the analysis of spectropolarimetric data to detect changes in the photospheric and chromospheric magnetic fields during flares or CME lift off episodes and/or to determine the total available magnetic flux in an active region. A major step forward, however, requires both high spatial resolution, high cadence, and quasisimultaneous multi-layer measurements of the solar atmosphere. 
A possible solution to this challenge is the deployment of instruments capable of fast scanning different spectral lines with quasi-monochromatic polarimetric imaging.

The Italian scientific community studied a Fabry-Pérot based instrument for interferometry and polarimetry from space (Berrilli et al., 2010). A NIR telescope equipped with a panoramic interferometer based on a double pass single etalon would combine high-spectral resolution with short exposure times and a large field of view, as well as the ability to work in polarized light. Observations could be performed over a large wavelength range, in the red part of the spectrum with high spectral resolution, high temporal resolution, high wavelength stability and polarimetric accuracy.

\subsubsection{X-ray polarimetric imaging to reveal the nature of the polarization of hard X-ray sources in the solar atmosphere}

X-ray polarimetry of solar flares could be a new tool for novel research in the field of Space Weather science (Hardi et al., 2012; Berrilli et al., 2015), being a powerful diagnostic of the properties of the magnetic field and of the plasma acceleration regions in the Sun's atmosphere. Indeed, direct electromagnetic information from the reconnection region associated with the CME is likely the fastest alert of the entire event.

It is now well-established that during solar flares magnetic field energy dissipation within the Sun's atmosphere and particle acceleration take place (Lin \& Hudson, 1976). The spectrum of solar flares in the X-ray energy band is characterized by the soft X-ray component (up to $\sim 15-20 \mathrm{keV}$ ) and the hard X-ray component; during the event lifetime both components evolve making the solar flare a dynamical source of Space Weather. The continuum of the soft spectrum is due to thermal bremsstrahlung (Peres et al., 1987; Fludra et al., 1995; Battaglia et al., 2009), whereas hard X-ray emission is due to non-thermal bremsstrahlung, when precipitating electrons hit the lower and denser layers of the solar atmosphere (Lin and Hudson 1976). We note that below $8 \mathrm{keV}$ the continuum of Bremmstrahlumg has overimposed a huge flux of emission lines from a plasma in thermal equilibrium or almost in equilibrium. Hard X-ray emission may originate from the coronal loop top or the region of the flare footprints (Krucker et al., 2008). To distinguish the contribution in the emission of each source region, X-ray polarimetry imaging techniques have to be applied (Fabiani \& Muleri, 2014).

Bremsstrahlung is responsible for a large fraction of the flare emission and, in case of particle beaming, it gives rise to a significant linear polarization (Fabiani \& Muleri, 2014). In particular, solar flare models that assume an anisotropic distribution of accelerated electrons in an ordered magnetic field predict that the hard X-Ray non-thermal Bremsstrahlung component should be highly polarized (Brown et al., 1974; Emslie \& Vlahos, 1980; Zharkova et al., 1995; Charikov et al., 1996), with a polarization degree as high as $40 \%$ at $20 \mathrm{keV}$ (Zharkova et al., 2010). Moreover, Emslie \& Brown (1980) proposed a model of X-ray thermal emission expected to be polarized at a lower level. Based on the aforementioned considerations, it is clear that the precise measurement of the hard X-ray polarization in solar flares can provide important constraints for the estimation of the geometry of the hot plasma source region, given a known magnetic field configuration. This is a real challenge for both basic physics and Space Weather science. In the context of the latter, such an information can be an important input for the related particle acceleration models. The localization of solar flares onto the solar disc is provided to find the relation between the polarization measurement and the observation line of sight. Polarization measurements of X-ray emission from solar flares will allow to:

- obtain for the first time an unambiguous detection of X-ray polarized radiation from solar flare emission;

- propose the X-ray polarimetry as a new tool to study magnetic reconnection;

- put strong constrains in plasma acceleration models in the solar atmosphere;

- test (and improve) current models for solar flare emission mechanisms based on their compatibility with polarimetric measurements.

The timing for this step forward is particularly good. Stellar $\mathrm{X}$-ray polarimetry has been rejuvenated thanks to the development of Gas Pixel Detectors (GPDs). The functionality of these new devices is based on the photoelectric effect. After 40 years, the launch of the Imaging X-Ray Polarimetry Explorer (IXPE) mission of NASA is scheduled for April 2021. The focal plane of IXPE is entirely designed, built and tested in Italy by INAF$I A P S$ and INFN-Pisa; all the expertise on this kind of measurement is resident in Italy. For X-ray flare polarimetry, an extension to the higher energy range would be needed. Such a scenario has been already studied in the past years, when a prototype model was tested at INAF-IAPS. Since December 2018, a CubeSat built by the Tsing Hua University, hosting a lowenergy GPD built in Italy, is successfully operative on a Sunsynchronus orbit. A similar nanosatellite, with a GPD tuned to higher energies, can be built in relatively short times and provide the first positive detection of polarization in solar flares, to be correlated with other parameters, including those characterizing CMEs.

\subsubsection{Energetic Neutral Atom imaging for investigating geomagnetic activity, Planetary Space Weather and energetic particle sources on the Sun}

ENA imaging is a consolidated technique which has an interesting potential in Space Weather Science since it can be employed as a complementary diagnostic tool for the spatial mapping of solar storm-driven magnetospheric disturbances, the regions of interaction between solar wind/ SEPs and planetary exospheres, and source regions of solar flares and CMEs that are associated with emission of protons with energy typically below $10 \mathrm{MeV}$. ENA imaging hence is a potential scientific tool in the context of both circumterrestrial and planetary Space Weather. We provide below the scientific rationale considering the expected add-on value that ENA imaging is likely to provide in Space Weather science.

\subsubsection{ENA imaging from low-altitude and medium/low-latitude orbits to monitor geomagnetic activity}

ENA fluxes produced through charge-exchange between the Earth's magnetospheric ions and the extended neutral 
geocorona or deeper exosphere are signatures of the (inner) magnetospheric activity, and can be correlated with Space Weather events, such as the geomagnetic storms and substorms, as well as the plasma sheet dynamics. Past successful missions have demonstrated the feasibility of the ENA imaging techniques in measuring such dynamic phenomena in the Earth's magnetosphere. Among the most successful ones are the Imager for Magnetopause-to-Aurora Global Exploration (IMAGE; Mitchell et al., 2003) and the Two Wide-angle Imaging Neutral-atom Spectrometers (TWINS; McComas et al., 2009) missions that demonstrated that it is possible to global view the storm/substorm dynamics. In fact, before the ENA era, it was not possible to obtain synoptic observations of the system with time resolution much shorter than the substorm time scale, so that a great improvement in the Space Weather investigation has been obtained since then. The TWINS mission enabled the three-dimensional visualization of the large-scale structures and dynamics within the magnetosphere for the first time (Goldstein \& McComas, 2013). In the context of Space Weather, through the composition-separated TWINS images, it has been possible to provide further evidence that the $\mathrm{O}^{+}$ring current is strongly intensified during the main phase of the storm and it survives much longer into recovery than $\mathrm{H}^{+}$(Valek et al., 2013).

Remote sensing of ENAs in the Earth's environment provides detailed information on the ring current and shock front plasma populations at energies between $1-3 \mathrm{keV}$ and up to $100 \mathrm{keV}$. Such ENA emissions are also generated in the cusps at high latitudes, and are in general subject to the space environment variability. Up-to-now the ENA imaging technique has been used mainly from high altitude polar orbiting spacecraft, which do not allow a continuous and systematic monitoring, neither the discrimination of the particle latitude distribution, an important parameter in the context of Space Weather. Instead, the continuous ENA monitoring from LEO spacecraft in medium/low latitude orbit, would permit the wide-field ENA imaging of different magnetospheric regions providing at the same time maps of both the ring current and shock front plasma regimes and their intensification during storms, a diagnostic for geomagnetic activity. Recently, on the basis of observations with ion analyzers on board the POES satellites, it was demonstrated that ENAs could be indeed monitored from low altitudes (Søraas \& Sørbø, 2013). The measurement of the spatial variability of the ring current and shock front regions, in terms of ENA maps, is an important feedback for the global understanding of the evolution of a geomagnetic storm, which in turn can affect technological systems. Consequently, in the context Space Weather science, the combined ENA, groundbased and in situ measurements (e.g., magnetic fields, plasma) during periods of intense geomagnetic activity can contribute in the improvement of current dynamic models of the space environment.

\subsubsection{ENA imaging as a tool for monitoring planetary Space Weather}

Within planetary environments the ENAs are produced by different processes: through charge exchange reactions (similar to those in the terrestrial magnetosphere), when solar wind and magnetospheric ions interact with exospheric neutral atoms of planets or moons; through back scattering when the solar wind ions reach a surface and are reflected back; through atmospheric or surface sputtering when the solar wind impacts exobases or surfaces. They are expected to be highly variable during Space Weather events being a kind of remote sensing of the parent ion population. Charge exchange ENA fluxes (energies between 1 and $100 \mathrm{keV}$ ) have been successfully detected at Earth (Mitchell et al., 2003; McComas et al., 2009), Jupiter (Mauk et al., 2003), Saturn (Krimigis et al., 2005) and Titan (Garnier et al., 2007), Mars (e.g., Futaana et al., 2006; Mura et al., 2009), and Venus (Galli et al., 2008) opening a perspective for global tracking of the variability of energetic ion propagation in the Solar System.

\subsubsection{ENA imaging for the investigation of SEP sources on the Sun}

Remarkably, ENAs with energies up to a few $\mathrm{MeV}$ have been identified in 2006 by the Reuven Ramaty High Energy Solar Spectroscopic Imager (RHESSI) on board the Solar TErrestrial RElations Observatory (STEREO) (Mewaldt et al., 2009). Although charge-exchange reactions between the energetic protons and coronal populations close to the Sun seems to be the physical mechanism resulting in ENA generation, the physical processes responsible for the observed emissions are not yet fully understood. It follows that, this possible ENA observation may potentially open a new and interesting window for SEP detection close to the Sun, allowing for the first time Space Weather investigations based on measurements of this kind.

INAF-IAPS has a strong expertise in designing ENAdetectors (see Sect. 2.3.3). Based on this experience, the Italian community can potentially extend its expertise to the design and implementation of high-energy ENA detectors to effectively monitor the ring current shock front and cusp plasma evolution during periods of intense Space Weather activity.

\subsubsection{Particle detectors to measure the radiation environment in or outside the Earth's magnetosphere}

Very few measurements are available as of today to measure the spectra, composition, and incoming direction of highly penetrating particles $(>\sim 100 \mathrm{MeV} / \mathrm{n})$ in deep space, outside the Earth's magnetosphere. The new US Geostationary Operational Environmental Satellite-R (GOES-R) series $^{3}$ carries on board its Space Environment In Situ Suite (SEISS) a particle sensor with a $>500 \mathrm{MeV}$ channel, for monitoring highly penetrating radiation that can reach aircraft altitudes. Currently, there are two of this new series in orbit: GOES-16 and GOES-17, which, however, are inside the magnetosphere most of the time. Previous instruments could not perform these measurements either because they were not operating in deep space (e.g., PAMELA, AMS-02, HEPD) or because the technology used was not capable of measuring the particle energy above a few 100s MeV/n (e.g., SOHO, ACE).

Based on the heritage of the successful program in LEO, Italy has the capability to assess the relevance of the scientific case, both in Space Weather and cosmic ray physics, as well as to design and realize the payload for a long term mission studying radiation environment in deep space and/or close to other planets of our Solar System. To fill the GeV observational gap between magnetic spectrometers (such as PAMELA and

\footnotetext{
3 https://www.ngdc.noaa.gov/stp/satellite/goes-r.html
} 
AMS-02) and ionization/absorption instruments (such as CRIS or EPHIN), and to pursue long-term programs of radiation monitoring in deep space, the Penetrating particle ANalyzer (PAN) concept has been recently proposed (Wu et al., 2019). The PAN experiment is a modular magnetic spectrometer based on novel layout, with compact size $(\sim 40 \mathrm{~cm})$, limited mass $(20 \mathrm{~kg})$, and reduced power budget $(20 \mathrm{~W})$. With PAN, particle identity and momentum measurements are operated by means of a high-field permanent magnet Halbach arrays, silicon strip and pixel detectors, and fast scintillators read by silicon photomultipliers.

The instrument is designed to achieve a high dynamical response to charged particles and nuclei $(Z=1-26)$ with fast acquisition rate $\left(\sim \mathrm{MHz} / \mathrm{cm}^{2}\right)$, large geometric factor $\left(\sim 10 \mathrm{~cm}^{2}\right.$ sr), and wide energy range ( $\sim 0.1-20 \mathrm{GeV} /$ nucleon). Thanks to the adopted modular design, a mini-PAN version, with a weight of $\sim 5 \mathrm{~kg}$, could be easily integrated into a suite of energetic particle monitoring system covering the energy range from $\sim 10 \mathrm{MeV} / \mathrm{n}$ to $\sim 10 \mathrm{GeV} / \mathrm{n}$ which is crucial in terms of Space Weather monitoring.

It is of high importance to develop radiation detectors specifically intended for the concurrent study of the SEP radiation inside and outside a (deep) space vessel, to validate transport models, to investigate the role of precursors in the SEP event evolution, to monitor the incoming SEP spectrum, and to prepare for now-casting strategies for deep space exploration. In a wider Space Weather context and in view of human space exploration, these measurements should also be managed by smart systems to support the crew decision process during emergencies due to SEPs.

\subsubsection{In-situ measurements of plasma properties}

In the heliosphere, fundamental physical processes operate to control the momentum and energy transport and, eventually, the energy dissipation and particle acceleration, which are at the basis of Space Weather phenomena. It is worth mentioning a few relevant examples. In the highly ionized space plasma, turbulent fluctuations are responsible for the transport of energy from large to small scales, with consequent energy dissipation and particle acceleration (Bruno \& Carbone, 2013). At shocks, plasma bulk flow energy is converted into heat and, again, associated particle acceleration to high energy occurs (Treumann, 2009). Magnetic reconnection converts stored magnetic energy into plasma kinetic and thermal energy and enables the plasma mixing between originally separated regions. The magnetic topology reconfiguration following reconnection has global scale effects on the dynamics of a planetary environment. Thus, fundamental processes as the ones mentioned above are at the origin of key phenomena related to Space Weather such as the particle acceleration to high energies, plasma heating and system reconfiguration at global scales. Understanding the physics underlying Space Weather phenomena is mandatory, since it permits to develop better forecasting models. To reach this goal, the first step is to characterize the properties of individual plasma species (electrons, protons, and other ions) and of the magnetic and electric fields in the space environment.

The best opportunity to acquire knowledge on the plasma environment is using measurements collected in situ by probes carried on board spacecraft in the interplanetary space, where a wide range of different plasma conditions can be encountered.
In particular, the near-Earth environment, is a preferred place in this respect, due to ease of access and maximum available data rates (and hence high temporal and spatial resolution). Indeed, a wealth of high quality, multipoint plasma and fields observations by the ESA Cluster and the NASA THEMIS and MMS missions in the magnetosphere, and by the ESA Swarm mission in the ionosphere, provided major add-ons in science, enabling great advances in the characterization of the physical mechanisms occurring in all the key regions of the geospace. All the new findings, e.g., about reconnection configuration at the magnetopause and about the properties of bursty bulk flows and how they interact with the inner magnetosphere, should be now incorporated into Space Weather models (Eastwood et al., 2017). Moreover, efforts should be devoted to joint-data analyses exploiting systematically the whole data sets provided by such missions and the ground based observations.

Notably, the remarkable discoveries and the lessons collected during the last two decades of in situ observations have also highlighted the strong need for new measurements in terms of enhanced time and phase resolution of particles detection (Vaivads et al., 2016) and/or of multiple point, multi-scale plasma observations in the near-Earth space (e.g., Schwartz et al., 2009). A substantial leap forward would be driven by such observations in our comprehension on how energy is processed by fundamental plasma processes, like turbulence and shocks, and how particle are energized in space plasmas, enabling progresses in many Space Weather topics. Ion and electron three-dimensional velocity distribution functions in the thermal energy range are typically acquired using electrostatic analyzers of top-hat type. These instruments are the ultimate and essential detectors when high quality measurements of particle distribution functions are needed, for missions dedicated to study solar wind and magnetospheric plasmas. Instruments of this kind are on-board the Cluster mission and compose the SWA plasma suite on board the ESA's Solar Orbiter mission. In the framework of international collaborations, INAF-IAPS has acquired a solid experience in the field of plasma data reduction and scientific analysis. Moreover, the Italian community has developed a strong expertise in the Digital Processing Units that govern the plasma suites and process the particle measurements on-board. In addition, INAF-IAPS has expertise in the design and testing of electric field detectors, using dedicated facilities as the Plasma Chamber. In conclusion, regarding the future perspectives and needs, the Italian know-how can be used in the framework of new initiatives in terms of new space missions investigating space plasma physics and Space Weather, as well as the exploitation of the available data.

In conclusion, the Italian scientific community has great interest to participate in new, high level, initiatives fostering advance in the science enabling Space Weather. A substantial leap forward will be made after the enhancement of time and phase resolution of plasma measurements (Vaivads et al., 2016) and/or the exploitation of multiple point plasma observations in the near-Earth space (Schwartz et al., 2009).

\subsubsection{Space Weather studies with stratospheric balloons}

Since the Sun is the driver of Space Weather, forecasters need a 3-D view of the Sun and its atmosphere as a foundation 
for effective predictions. Currently, Space Weather forecasts are based on the analysis of observations from a single region in height of the solar atmosphere, thus limiting our predictive capabilities. Instruments that would provide high-cadence observations from two different heights could be very useful for advancing our insights in and understanding of the evolution of several features of the solar atmosphere.

Simultaneous 2-D magnetograph images of the magnetic and velocity fields at the two heights will allow unprecedented studies of both the magnetic field re-configuration, which causes flares and CMEs, and the local plasma dynamics in the atmosphere. The data will also enable a study of the dynamics of the sub-surface structure in the vicinity of solar active regions. A balloon mission that would demonstrate the scientific capability of MOF-based Doppler/magnetographs when used in the space environment would address key issues in preparation for future flight opportunities. These missions put severe constraints on the mass and volume available for instrumentation, and the ability to employ on-board data reduction and compression to significantly reduce the required data return bandwidth.

Coronagraph images on board a balloon mission may also provide useful information for the study of Space Weather phenomena. An internally-occulted coronagraph of the polarized broad spectral band K-corona and of the narrow spectral band polarized emission of the coronal "Green-line" at $530.3 \mathrm{~nm}$ (such as the ones used for ground-based observations, e.g., AntarctiCor, see Sect. 2.2.2) can be installed on a high-altitude (i.e., $\sim 30 \mathrm{~km}$ ) stratospheric balloon. The absence of atmospheric scatter for coronal observations from such a platform would considerably increase the instrument sensitivity. Such a coronagraph would then be very well suited for measuring with highcadence spectral-imaging, the wave properties in corona, and their possible contribution to the coronal heating and to the solar wind acceleration.

\subsection{Development of a national scientific Space Weather data centre - the ASI Space Weather Infrastructure (ASPIS)}

ASPIS at a glance: The development of ASPIS is one of the highest priorities within the current roadmap. The proposed scientific Space Weather data center will be an important reference point for national scientific research as it will provide high-quality interdisciplinary data and global information relevant to Space Weather science in an extent that currently is not covered by any other Italian infrastructure. ASPIS will enable interdisciplinary research and innovation integrating the activities of at least seven Italian science communities interested in Space Weather (i.e., solar physics; solar-terrestrial physics; geomagnetism; ionosphere and upper atmosphere physics; Planetary Space Weather science; galactic cosmic ray physics; technological and biological impacts study communities) in a unique coherent science data center, providing a broad set of parameters in the Space Weather domain. Given its multidisciplinary nature, we propose to host ASPIS at the ASI Space Science Data Center (SSDC).

Within ASPIS, at a best effort basis, duplication of existing international efforts will be avoided. Indeed, overlap in focus of existing activities with the ASPIS science goals may result in substantial benefits as long as the research is synergistic and not duplicative. ASPIS aims at motivating synergies and collaboration opportunities among researchers interested in Space Weather. Such synergies between different science teams could be essentially facilitated by an efficient access to multidisciplinary data. Moreover, through the widening of the data access beyond the communities with direct interest in Space Weather, unprecedented scientific achievements can be obtained. We provide below our recommendations considering the development of ASPIS and we underline that our suggestions may well be modified and/or integrated in the next years on the basis of possible new top level needs. The suggested pathways, therefore, are only potential options.

Vision, Objectives, Users and Products: Individual research teams in Italy have always provided a large component in Space Weather science progress and continue to do so. However, as knowledge on the complexity of Space Weather grows, the possibility of breakthrough science in the next years requires the interaction between groups that include observers, modelers, theoreticians, laboratory, and computer scientists. ASPIS aims at being the central node of the Italian research activities related to Space Weather, increasing the excellence in circumterrestrial and planetary Space Weather research and motivating the development of solutions to current science challenges. The proposed national scientific Space Weather data center ASPIS will offer an excellent opportunity to host, organize and visualize the interdisciplinary Space Weather data produced by the Italian community, as well as scientific products generated by tools already developed by science teams but still not widely distributed among all interested users. The development of ASPIS is an important step for addressing a number of scientific questions related to Space Weather (see also Sect. 3).

The key goal of ASPIS is to disseminate high-quality interdisciplinary Space Weather data to support scientific research in Italy in the field. Based on the above, the main ASPIS objectives can be summarized as follows:

- to provide efficient storage, sophisticated organization, and explanative visualization of interdisciplinary Space Weather data and to offer user-friendly data access and related documentation;

- to provide scientific products derived from the original data to be further used in scientific Space Weather models and applications;

- to foster the generation of interdisciplinary data products that can potentially provide relevant inputs for advanced scientific Space Weather models;

- to provide test beds for forecasting models to be run on historical data;

- to support the promotion of education and awareness in Space Weather;

- to maintain a long-term relationship with SWICo also through the organization of Space Weather dedicated workshops and meetings.

ASPIS will provide access to data essential to a wide range of communities that can be further expanded with a long-term strategy. Such user communities are:

- Space Weather research communities (observational, experimental, modeling, scientific payload development communities) with special interest in long-term research programs; 


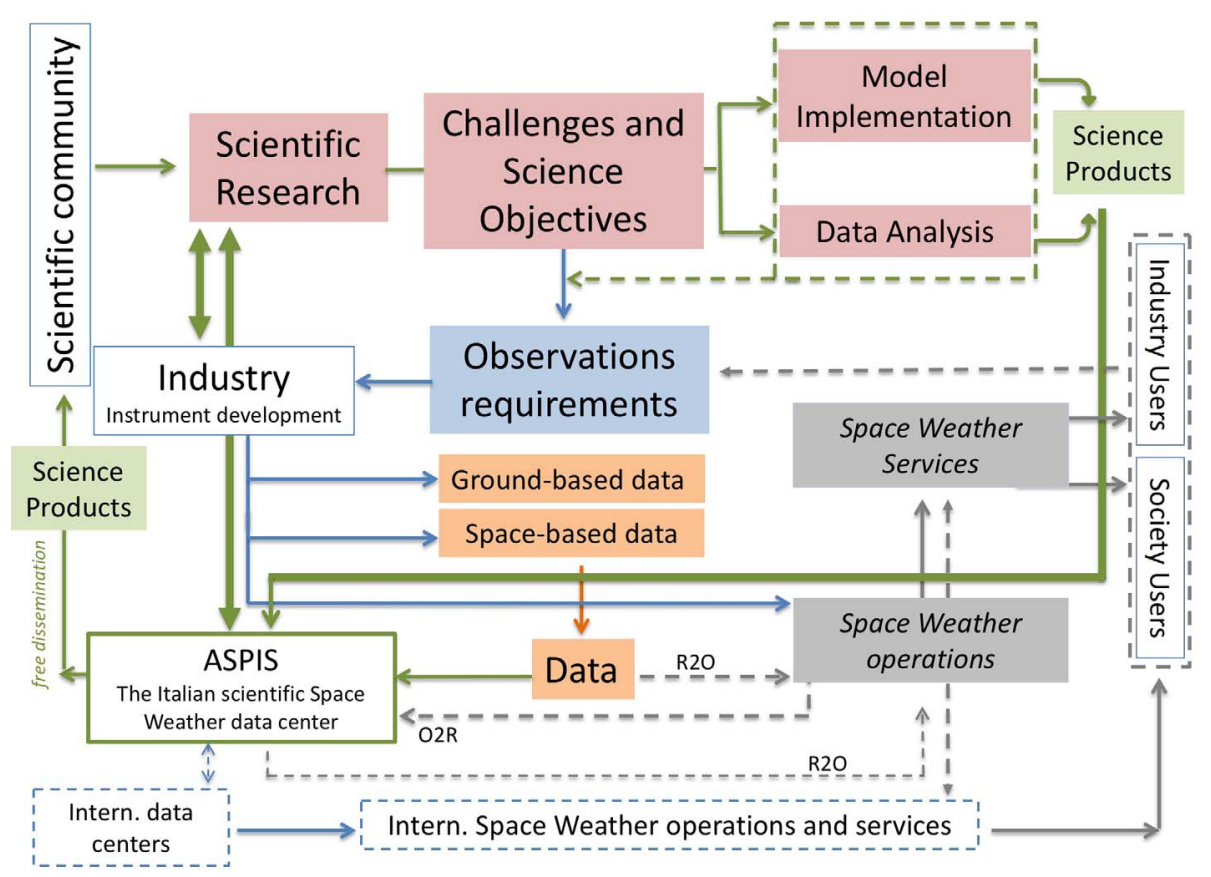

Fig. 6. Framework with the logical associations corresponding to the proposed roadmap for Space Weather science. The national scientific Space Weather data center ASPIS can play a major role in hosting Space Weather data archives and related tools, and in offering a centralized entry point to coherently access Space Weather related resources, providing at the same time a fruitful and collaborative environment. Synergistic coordination of national research in the field of Space Weather, based on the use of all relevant data sources, theory and modeling would guarantee the avoidance of unnecessary duplications and the amplification of synergies through targeted partnerships. Policy and education embrace the whole structure.

- Environmental science research communities from neighboring fields (e.g., climate study, geosciences, aeronomy, astrophysics, astrobiology);

- Instrument manufactures and industries with interest in the development, testing, prototyping and demonstration of sensors;

- Mission proposal teams interested in validation and development of new mission scenarios;

- Operational service developers in the field of Space Weather monitoring and forecasting.

In Figure 6, we present the conceptual design of the proposed roadmap for national Space Weather research, with the future scientific data centre ASPIS being its main core. All data and tools hosted in ASPIS should be made freely accessible to scientists, with the condition that proper acknowledgments about the sources is explicitly given in the works produced by these users. The establishment of a dedicated open access data dissemination policy describing the roles and responsibility of data providers as well as the rules and guidelines that the users should follow will be a fundamental task to be performed during the ASPIS implementation phase. Such a policy should aim at making the data compliant with the FAIR principles for science data products: findable, accessible, interoperable, and reusable. In order to achieve this goal, we envisage the need for data identifiers, suitable open access licenses, use of already existing standard data formats and definition of missing standards, comprehensive documentation and data products description. It is underlined that an ASPIS open access data dissemination policy will allow scientific progress at large. Moreover, ASPIS will benefit early-career researchers providing easy access both to multi-disciplinary Space Weather data and related documentation.

The Principal Investigators (PIs) providing data to ASPIS are responsible for the acquisition and delivery of high-reliable and quality controlled information. ASPIS will be responsible for handling the data, which will be of very different types (e.g., images, spectra, fluxes). The ASPIS team will compile and archive the data, providing access, via the ASPIS data portal, to well documented Space Weather data and data products, including tools for visualization, comparative analysis and joint investigations. Being a tool for science, ASPIS aims at maintaining and increasing the availability of Space Weather data to all interested users.

In summary, ASPIS will benefit scientists working in the field of Space Weather by providing open-access interdisciplinary data, added-value data products, and data procedure inter-comparisons for conducting excellent research and creating new scientific knowledge.

Technical issues: Space Weather research often requires the use of data originating from different instrument platforms, ground-based and space-based. Due to this reason, optimization of observational coverage by coordinated measurements by the existing ground-based and space resources, standardization of the meta-data - as far as possible - and harmonization of the access to data archives, are necessary. The architecture of ASPIS should be compliant with international frameworks (e.g., the 
NOAA's Space Weather Prediction Center, the ESA Expert Service Centers, etc.). Special attention should be paid in the design of ASPIS, which should not neglect the possible need for future establishment of Space Weather services requiring the accessibility to real-time (or near-real time) data. The retrospective data to be hosted by ASPIS will be fundamental for interdisciplinary research purposes (Plainaki et al., 2017, 2018). Hence their correct processing, efficient storage, sophisticated organization, and visualization are fundamental parameters for the preserving the overall scientific value of the data. In this context, the efficient data archiving within ASPIS will guarantee the center's long-term utility. ASPIS, therefore, will have an important scientific role but at the same time its technical basis should guarantee the possibility to provide at a future moment real-time (or near real-time) data to be potentially "ready-to-serve" for applications developed by different national institutions with interest in Space Weather.

To achieve the efficient operation of ASPIS, we recommend the following actions:

- the effective collaboration between different teams through research investigations focused on specific scientific case studies of interdisciplinary nature;

- the optimization of observational coverage by coordinating the observations of the existing ground-based and space resources;

- the existence of collaboration studies for the development of new instrumentation based on advanced technology benefitting from the heritage of past projects;

- the building of future test beds where the Space Weather model development can be supported by coordinated and inter-disciplinary observations;

- the harmonization - as far as possible - of the access to data archives;

- the development of advanced techniques for big data processing.

Current advances in computational capabilities can be a resource for ASPIS in view of further developments in Space Weather modeling and data-analysis. Expertise in computer science, algorithm development, artificial intelligence techniques (e.g., machine learning), problem solving, and data visualization can definitely integrate the potential of scientific teams. In this context and with the reference to new projects special attention should be paid to avoiding unnecessary duplications and to amplifying synergies through targeted partnerships.

ASPIS and international initiatives: Several international entities have special interest in Space Weather and its related scientific aspects. For example, the International Space Weather Initiative (ISWI) ${ }^{4}$ is a program of international cooperation aiming to advance Space Weather science by a combination of instrument deployment, analysis and interpretation of Space Weather data from the deployed instruments in conjunction with space data, and communicate the results to the public and students. The goal of ISWI is to develop the scientific insight necessary to understand the science, and to reconstruct and forecast near-Earth Space Weather, including instrumentation,

\footnotetext{
4 http://www.iswi-secretariat.org/
}

data analysis, modeling, education, training, and public outreach. Its success depends on unrestricted flow of data across geo-political and organizational boundaries. In this view, the role of the proposed ASPIS scientific center could become of significant importance also within such an international context, since the related activities are likely to contribute significantly in the overall progress in Space Weather science, strongly supported by ISWI.

Moreover, we note that ESA has promoted the development of a Space Weather virtual modeling center in Leuven in the framework of the Space Situation Awareness (SSA) program. Also in this context, the analysis of the ASPIS data is expected to result in additional scientific returns in the field of Space Weather at international level; indeed, the expected outputs of interdisciplinary data analyses within ASPIS are likely to be of help during international efforts aiming at the coupling of the state of the art physics-based models into an operational environment. More specifically, the expected return from scientific activities in all the Space Weather-related scientific fields are likely to provide new insights, motivating further synergetic approaches also at international level.

In the context of the efforts of the COSPAR panel on Space Weather, our proposal for the development of a national scientific Space Weather data center comes timely, fulfilling one of the most important needs discussed in the related documentation. In particular, the COSPAR and ILWS roadmap recommends that funding agencies require development of Space Weather data archiving, data search, and data access plans and, most importantly, coordinate any needed reprocessing of historical datasets to make them generally available in a standard way. From our side, we embrace these recommendations, not only promoting the efficient archiving of Space Weather data in Italy, but also motivating their further use and elaboration for scientific modeling and comprehensive analysis aiming at the understanding of the related physical phenomena at large. Last, we strongly believe that our efforts focused on the development of ASPIS will have a positive impact also in the establishment and coordination of international active networks and topical focused collaborations, potentially within the International Space Weather Action Teams (ISWAT) proposed by COSPAR. Indeed, the organization of the available material within ASPIS into targeted science cases will facilitate possible feedback exchange with the entire international Space Weather community, motivating innovation to advance our current understanding in the field.

In summary, ASPIS can have a key role in Space Weather activities coordinated also at international level, offering interdisciplinary data archives and scientific model outputs to be used in a series of test applications.

ASPIS in view of future development of national Space Weather applications: The proposed development of ASPIS can be an important propaedeutic step for future operational activities in Space Weather to be developed by different institutions with interests in the field. In this context, the current design and structure of ASPIS, should guarantee the possibility to include at a future moment accessibility to real-time (or nearreal time) data. In such a case, targeted $\mathrm{R} 2 \mathrm{O}$ pathways are a necessary requisite. ASPIS will be a resource for the national research institutes and the national industry that wants to develop new tools. 


\subsection{Teaming and Collaboration}

The coordination of scientific efforts relevant to Space Weather and the possibility of effective exchange of the related material, are major needs in view of future progress in the field of Space Weather science. Very often, such scientific efforts are scattered with respect to each other and short-term, and the visibility of the overall outcome of the related projects is somehow limited. To maximize the return of the scientific projects related to Space Weather, efficient teaming and collaboration between different interested partners are necessary.

The proposed ASPIS aims at motivating synergies between different science teams with interest in the field of Space Weather and also to motivate innovation and new mission concept development. In view of the establishment of ASPIS, it is important to guarantee the existence of a fruitful and collaborative environment. The following recommendations are provided:

- implementation of an open Space Weather data and information policy;

- preparation of the future transition from scientific research models to actual Space Weather forecast models (definition of R2O pathways); within ASPIS, building of test beds to support data-based model development;

- consolidation of partnerships across the international Space Weather community to share lessons learned and to avoid unnecessary duplications;

- establishment of partnerships with the national and international geophysics, meteorology and planetary science communities to better understand coupling phenomena.

An important aspect related to all of the above recommendations is the possibility of technical information exchange. For example, the exchange of information on the state of the development of theoretical and data-driven modeling techniques could result in major improvements of both the existing and future capabilities. To this purpose, periodical meetings dedicated to the scientific reviewing of the developments and the discussion of potential upgrades during future developments would be very helpful.

\subsection{Education, training, and public outreach}

There are several academic courses in Italy designed to disseminate knowledge in the field of Heliophysics and Space Weather science. Such courses cover several thematic areas related to Space Weather providing fundamental information on the physics of the Sun and space plasmas. The solar wind and solar eruptive phenomena, the Earth's magnetosphere and its interaction with the solar wind and SEPs, particle propagation in the interplanetary space, planetary space weather, space instrumentation, are some of the discussed arguments.

A complete list of all national and international educational activities in the field of Space Weather science leaded by the Italian Space Weather community, continuously updated and integrated with state of the art material, goes beyond the scope of the current paper. Here we report some of the main recent activities in the field, underlying that the provided material is not exhaustive. Indicatively, we refer to the "Space Weather" course by UNIVAQ (Laurea Magistrale/Atmospheric Science and Technology), the "Space Physics", "Physics of the Circumterrestrial Space", and "Physics of the Magnetosphere" courses by UNIVAQ (Laurea Magistrale /Geophysics and Space), and the "Sun-Earth connection and Space Weather" course by UNICAT ( $\mathrm{PhD}$ in Physics course). Within the Master in Science and Space Technology organized by UNITOV, the "Sun and Space Climate Course" is held. Moreover, within the International School of Space Science (ISSS), organized by the Italian inter-University Consortium for Space Physics" (CIFS) ${ }^{5}$ and INAF, several courses related to Space Weather have been held. A course in "Meteorology and Climatology of Space" (Laurea Magistrale in Fisica) and a course in "Physics of SpaceGeospace Interactions" (Dottorato in Fisica) are held in UNITS.

Our recommendations in the field of education and training may be summarized as follows:

- to develop and provide access to educational material on both Space Weather science and Space Weather technological and biological impact information; create tutorials and Frequently Asked Questions sessions;

- to develop and maintain web structures for access to these materials;

- to design university undergraduate and master courses in Space Weather, with special emphasis on its relation with space activities;

- to design training courses in Space Weather for undergraduate and master students;

- to work on the collaboration among universities and, potentially, research institutes to promote Space Weather education;

- to work on the collaboration with the wider scientific community working in closely related scientific fields (e.g., planetary exploration, atmospheric physics, astrophysics).

Although there is an international public interest in Space Weather, many current websites often contain over-simplified information considering both the scientific and operational aspect of Space Weather. In the context of the current roadmap, we provide the following recommendations:

- guide Space Weather stakeholders and public educators towards qualitative information;

- generate outreach and simplified tutorials under the guidance of professional Space Weather experts.

Finally, we recommend further synergies between current educational and training activities in Italy and relevant international efforts. Such synergies are likely to favor the exchange of the related material and feedback in a continuous and coherent way, increasing the efficiency of the whole system.

\section{Conclusions}

To mitigate Space Weather, a deep understanding of the underlying physics, based also on the analysis of the available

\footnotetext{
${ }^{5}$ CIFS joins several Italian Universities active in the field of Space Science (UNICAT, UNIFI, UNIVAQ, UNIMI, UNIROMA1, UNITOV, UNITO, UNITS).
} 
interdisciplinary ground- and space-based measurements, is required.

The efficient synergy between ASI and the national scientific and industrial communities with interest in Space Weather has resulted in Italy's participation in numerous international efforts aiming at advancing our insights in and understanding of the mechanisms determining the Space Weather phenomena. Innovative instrumentation on board robotic Solar System exploration missions, designed and developed (either entirely or partially) in Italy, has provided important insights in circumterrestrial and planetary Space Weather science. In addition, ground-based observations have been an important resource for numerous studies in the field of Space Weather science.

In this paper, we provided a series of recommendations toward an in-depth understanding of the scientific aspects behind Space Weather, taking into consideration the existent observational and modeling capabilities supporting Space Weather research in Italy. Considering theoretical and observational recommendations, we identified three main Directions (Scientific data-driven modeling; Basic physics behind Space Weather; Space Weather now-casting, forecasting and impacts analysis) and we proposed specific approaches, also in a wider international context. We also discussed the study of space mission concepts and deployment of new instrumentation for obtaining key measurements to better address current open science questions in the field.

Given its interdisciplinary nature, Space Weather science requires coordination at all levels. To set the basis for improved Space Weather forecasting capabilities, significant advances in the underlying physics are required. At the same time, the loop between R2O and O2R should be supported in a coherent and efficient way. Future actions in the research fields related to Space Weather must incorporate both existing and new measurements of the entire system including data from groundbased networks and in situ space-based observations of the state and dynamics of the near-Earth space environment. We underline that the proposed recommendations may well be modified and/or integrated in the next years on the basis of possible new top level needs (decided at national or international context) hence the pathways we provided in this paper are only potential options.

In the context of a long-term strategy, we propose the development of a national scientific Space Weather data center, to be allocated in ASI's SSDC, to host both Space Weather data archives and related scientific tools. The so called ASI Space Weather InfraStructure (ASPIS) will function as a multidimensional tool for science being an important reference point for research as it will provide high-quality interdisciplinary data, scientific modeling tools, and global information relevant to Space Weather science, in an extent that is currently not covered by any other Italian infrastructure. ASPIS will give the possibility to integrate activities of at least seven Italian science communities interested in Space Weather in a unique coherent frame, providing a broad set of parameters in the Space Weather domain. ASPIS aims at motivating synergies and collaboration opportunities at national level, increasing the excellence in circumterrrestrial and planetary Space Weather research and motivating the development of solutions to current science challenges. Through the widening of the data access beyond specific communities unprecedented scientific achievements can be obtained. In particular, the ASPIS data, together with targeted advances in modeling, will be the key towards Space Weather forecasting capabilities. The proposed development of ASPIS, therefore, can be an important propaedeutic step for future activities in Space Weather.

ASPIS will benefit researchers in the field of Heliophysics and Space Weather science by providing interdisciplinary data, added-value data products, and data procedure inter-comparisons for conducting excellent research and creating new scientific knowledge. The centralized access to Space Weather data will enhance research performance increasing the possibility for large-scale research projects, training opportunities, and international collaborations. Moreover, the ASPIS data can be of great support during the preparation phases of possible future Space Weather missions or robotic Solar System exploration missions with objectives related to Space Weather. ASPIS will be a resource for the national research institutes, but also for the national industry that wants to develop new tools.

Last, we note that our recommendations and in particular our proposal for the development of a national scientific Space Weather data center are coherent with the efforts of the COSPAR panel on Space Weather. Recognizing the recommendations of the COSPAR and ILWS roadmap on Space Weather, not only we promote the efficient archiving of Space Weather data in Italy, but we motivate their further elaboration for scientific modeling - preferably through interdisciplinary collaborations - to understand the physics behind Space Weather. In a wider international context, therefore, the development of ASPIS will have a positive impact also in the establishment and coordination of international active networks and topical focused collaborations, facilitating the feedback exchange with the entire international Space Weather community and motivating innovation. The field of robotic Solar System exploration can greatly benefit from progress in the field of Space Weather both from a scientific and technological (e.g., spacecraft and instrument protection) point of view.

Acknowledgements. We are extremely grateful to Enrico Costa, Anna Milillo, Davide Grassi, Imma Donnarumma, and Eleonora Ammannito for their important suggestions and comments that helped to improve the overall quality of this paper. We would like to thank also the following colleagues and experts for their feedback and fruitful discussions in the context of this effort: Alberto Adriani, Vincenzo Andretta, Ester Antonucci, Roberto Bove, Alfredo Del Corpo, Dario Del Moro, Elisabetta De Angelis, Cristian De Santis, Piero Diego, Ilaria Ermolli, Silvano Fineschi, Patrizia Francia, Catia Grimani, Stefano Massetti, Alessandro Mura, Carlotta Pittori, Paolo Romano, Marco Romoli, Giuseppe Sindoni, Nicola Tomassetti, Valerio Vagelli, Massimo Vellante, Umberto Villante, and Francesca Zuccarello. The editor thanks three anonymous referees for their assistance in evaluating this paper.

\section{References}

Aboudarham J, Bentley RD, Csillaghy A. 2012. HELIO: a heliospheric virtual observatory. ASP Conf Ser 461: 255.

Adriani O, Barbarino GC, Bazilevskaya GA, Bellotti R, Boezio M, et al. 2015. Pamela's measurements of magnetospheric effects on 
high energy solar particles. Astrophys J Lett 801: 1. https://doi.org/ 10.1088/2041-8205/801/1/L3.

Adriani O, Barbarino GC, Bazilevskaya GA, Bellotti R, Boezio M, et al. 2016. Time dependence of the electron and positron components of the cosmic radiation measured by the PAMELA experiment between July 2006 and December 2015. Phys Rev Lett 116: 241105 . https://doi.org/10.1103/PhysRevLett.116.241105.

Adriani A, Filacchione G, Di Iorio T, Turrini D, Noschese R, et al. 2017. JIRAM, the Jovian Infrared Auroral Mapper. Space Sci Rev 213: 393. https://doi.org/10.1007/s11214-014-0094-y.

Aguilar M, Ali Cavasonza L, Alpat B, Ambrosi G, Arruda L, et al. 2018. Observation of fine time structures in the cosmic proton and helium fluxes with the alpha magnetic spectrometer on the international space station. Phys Rev Lett 120: 051101. https://doi.org/10.1103/PhysRevLett.121.051101.

Aguilar M, Ali Cavasonza L, Ambrosi G, Arruda L, Attig N, et al. 2018. Observation of complex time structures in the cosmic-ray electron and positron fluxes with the alpha magnetic spectrometer on the international space station. Phys Rev Lett 120: 051102. https://doi.org/10.1103/PhysRevLett.121.051102.

Albanese C, Rodriguez F, Ronchini R, di Rollo S, Berrilli F, et al. 2018. The ionosphere prediction service, space weather of the heliosphere: processes and forecasts. Proc Int Astron Union 335: 352-354. https://doi.org/10.1017/S174392131800025X.

Alfonsi L, Spogli L, Pezzopane M, Romano V, Zuccheretti E, et al. 2013. Comparative analysis of spread-F signature and GPS scintillation occurrences at Tucumán, Argentina. J Geophys Res 118: 4483-4502. https://doi.org/10.1002/jgra.50378.

Alfonsi L, Cilliers PJ, Romano V, Hunstad I, Correia E, et al. 2016. First observations of GNSS ionospheric scintillations from DemoGRAPE project. Space Weather 14: 704-709. https://doi. org/10.1002/2016SW001488.

Antonucci E. 1994. SOHO contribution to the understanding of mass supply and flows in the solar corona. Space Sci Rev 70: 149. https://doi.org/10.1007/BF00777859.

Antonucci E, Fineschi S, Gardiol D, Noci G, Romoli M, et al. 2000. Ultraviolet and visible-light coronagraph for the solar orbiter mission. Proc SPIE 4139: 378-389. https://doi.org/10.1117/ 12.410536.

Antonucci E, Romoli M, Andretta V, Fineschi S, Heinzel R, Moses JD, et al. 2019. Metis: the Solar Orbiter visible light and ultraviolet coronal imager. $A \& A$. Forthcoming article. https://doi.org/ 10.1051/0004-6361/201935338.

Armano M, Audley H, Baird J, Bassan M, Benella S, et al. 2018. Characteristics and energy dependence of recurrent galactic cosmic-ray flux depressions and of a Forbush decrease with LISA Pathfinder. ApJ 854: 542-565. https://doi.org/10.3847/1538-4357/ aaa774.

Asensio Ramos A, Trujillo Bueno J, Landi Degl'Innocenti E. 2008. Advanced forward modeling and inversion of stokes profiles resulting from the joint action of the Hanle and Zeeman effects. ApJ 683: 542-565. https://doi.org/10.1086/589433.

Aslam OPM, Bisschoff D, Potgieter MS, Boezio M, Munini R. 2019. Modeling of heliospheric modulation of cosmic-ray positrons in a very quiet heliosphere. Astrophys J 873: 70. https://doi.org/ 10.3847/1538-4357/ab05e6.

Bak-Steslicka U, Gibson SE, Fan Y, Bethge C, Forland B, Rachmeler LA. 2013. The magnetic structure of solar prominence cavities: new observational signature revealed by coronal magnetometry. ApJL 770: L28. https://doi.org/10.1088/20418205/770/2/L28.

Battaglia M, Fletcher L, Benz AO. 2009. Observations of conduction driven evaporation in the early rise phase of solar flares.
A\&A 498(3): 891-900. https://doi.org/10.1051/0004-6361/ 200811196.

Belehaki A, James S, Hapgood M, Ventouras S, Galkin I, Lembesis A, Tsagouri I, Charisi A, Spogli L, Berdermann J, Häggström I. 2016. The ESPAS e-infrastructure: access to data from near-Earth space. Adv Space Res.58: 1177-1200. https://doi. org/10.1016/j.asr.2016.06.014.

Bergeot N, Witasse O, Le Maistre S, Blelly P-L, Kofman W, Peter K, Dehant V, Chevalier J-M. 2019. MoMo: a new empirical model of the Mars ionospheric total electron content based on Mars Express MARSIS data. J Space Weather Space Clim 2019(9): A36. https://doi.org/10.1051/swsc/2019035.

Berrilli F, Bigazzi A, Roselli L, Sabatini P, Velli M, et al. 2010. The ADAHELI solar mission: investigating the structure of Sun's lower atmosphere. Adv Space Res 45: 1191-1202. https://doi.org/ 10.1016/j.asr.2010.01.026.

Berrilli F, Soffitta P, Velli M, Sabatini P, Bigazzi A, et al. 2015. ADAHELI+: exploring the fast, dynamic Sun in the x-ray, optical, and near-infrared. J Astron Telesc Instrum Syst 1(4): 044006. https://doi.org/10.1117/1.JATIS.1.4.044006.

Berrilli F, Casolino M, Del Moro D, Forte R, Giovannelli L, et al. 2018. SWERTO: a Regional Space Weather Service. Space Weather of the Heliosphere: Processes and Forecasts. Proc Int Astron Union: IAU Symp 335: 348-351. https://doi.org/10.1017/ S1743921318000054.

Belov AV, Eroshenko EA, Kryakunova ON, KurtV VG, Geomagn Yanke G. 2010. Ground level enhancements of solar cosmic rays during the last three solar cycles. Geomagn Aeron 50: 21-33. https://doi.org/10.1134/S0016793210010032.

Bindi V, Corti C, Consolandi C, Hoffman J, Whitman K. 2017. Overview of galactic cosmic ray solar modulation in the AMS-02 era. Adv Space Res 60: 865-878. https://doi.org/10.1016/j.asr.2017. 05.025.

Bisi MM, Jackson BV, Hick PP, Buffington A, Clover JM, et al. 2010. Three-dimensional reconstructions and mass determination of the 2008 June 2 LASCO coronal mass ejection using STELab interplanetary scintillation observations. Astrophys $J$ Lett 715(2): L104-L108. https://doi.org/10.1088/2041-8205/715/2/ L104.

Bombardieri DJ, Michael KJ, Duldig ML, Humble JE. 2007. Relativistic Proton Production during the 2001 April 15 Solar Event. Astrophys J 665: 813-823.

Brown JC, Mcclymont AN, Mclean IS. 1974. Interpretation of solar hard X-ray burst polarisation measurements. Nature 247: 448-449. https://doi.org/10.1038/247448a0.

Bruno A, Bazilevskaya GA, Boezio M, Christian ER, de Nolfo GA, et al. 2018. Solar energetic particle events observed by the PAMELA mission. Astrophys J 862: 2. https://doi.org/10.3847/ 1538-4357/aacc26.

Bruno R, Carbone V. 2013. The solar wind as a turbulence laboratory. Living Rev Solar Phys 10(2): 208. https://doi.org/10.12942/lrsp2013-2.

Bruno R, Telloni D. 2015. Spectral analysis of magnetic fluctuations at proton scales from fast to slow solar wind. ApJ. 811: 2. https://doi.org/10.1088/2041-8205/811/2/L17.

Buzulukova N, Fok MC, Roelof E, Redfern J, Goldstein J, et al. 2013. Comparative analysis of low-altitude ENA emissions in two substorms. J Geophys Res: Space Phys 118: 24-731. https://doi. org/10.1002/jgra.50103.

Carbary JF, Kane M, Mauk BH, Krimigis SM. 2014. Using the kappa function to investigate hot plasma in the magnetospheres of the giant planets. J. Geophys Res: Space Phys 119: 8426-8447. https://doi.org/10.1002/2014JA020324. 
Capparelli V, Zuccarello F, Romano P, Simoes P, Fletcher L, et al. 2017. $\mathrm{H} \alpha$ and $\mathrm{H} \beta$ emission in a $\mathrm{C} 3.3$ solar flare: comparison between observations and simulations. Astrophys $J$ 850: 1: https://doi.org/10.3847/1538-4357/aa9187.

Casolino M, Bidoli V, Minori M, Narici L, De Pascale MP, et al. 2006. Relative nuclear abundances inside ISS with Sileye-3/ Alteino experiment. Adv Space Res 37: 1685-1690. https://doi.org/ 10.1016/j.asr.2006.02.050.

Charikov JE, Guzman AB, Kudryavtsev IV. 1996. Hard X-ray emission of solar flares and non-stationary kinetics of electron beams. A\&A 308: 924-928.

Chisham G, Lester M, Milan SE, Freeman MP, Bristow WA, et al. 2007. A decade of the Super Dual Auroral Radar Network (SuperDARN): scientific achievements, new techniques and future directions. Surv Geophys 28: 33-109. https://doi.org/10.1007/ s10712-007-9017-8.

Consolini G, Grandioso S, Yordanova E, Marcucci MF, Pallocchia G. 2015a. Statistical and scaling features of fluctuations in the dissipation range during a reconnection event. Astrophys $J$ 804: 19. https://doi.org/10.1088/0004-637X/804/1/19.

Consolini G, Materassi M, Marcucci MF, Pallocchia G. 2015b. Statistics of the velocity gradient tensor in space plasma turbulent flows. Astrophys J 812: 84. https://doi.org/10.1088/0004-637X/ 812/1/84.

Contarino L, Zuccarello F, Romano P, Spadaro D, Guglielmino SL, et al. 2009. Flare forecasting based on sunspot-groups characteristics. Acta Geophys 57: 52-63. https://doi.org/10.2478/s11600008-0067-1.

Corti C, Potgieter MS, Bindi V, Consolandi C, Light C, et al. 2019. Numerical modeling of galactic cosmic ray proton and helium observed by AMS-02 during the solar maximum of Solar Cycle 24. Astrophys J 871: 253. https://doi.org/10.3847/15384357/aafac4.

De Franceschi G, Alfonsi L, Romano V. 2006. ISACCO: an Italian project to monitor the high latitudes ionosphere by means of GPS receivers. GPS Solut 10(4): 263-267. https://doi.org/10.1007/ s10291-006-0036-6.

De la Cruz Rodríguez J, Socas-Navarro H, Carlsson M, Leenaarts J. 2012. Non-local thermodynamic equilibrium inversions from a 3D magnetohydrodynamic chromospheric model. A\&A 543: A34. https://doi.org/10.1051/0004-6361/201218825.

De Michelis P, Consolini G. 2015. On the local Hurst exponent of magnetic field fluctuations: spatial distribution for different geomagnetic activity levels. J Geophys Res: Space Phys 120: 2691-2701. https://doi.org/10.1002/2014JA020685.

De Michelis P, Consolini G, Tozzi R. 2015. Magnetic field fluctuation features at Swarm's altitude: a fractal approach. Geophys Res Lett 42: 3100-3105. https://doi.org/10.1002/2015GL063603.

De Michelis P, Consolini G, Tozzi R, Marcucci MF. 2016. Observations of high-latitude geomagnetic field fluctuations during St. Patrick storm: Swarm and SuperDARN measurements. Earth Planets Space 68: 105. https://doi.org/10.1186/s40623-0160476-3s.

De Michelis P, Consolini G, Tozzi R, Marcucci MF. 2017. Scaling features of high latitude geomagnetic field fluctuations at Swarm altitude. J Geophys Res: Space Phys 122: 10548-10562. https://doi.org/10.1002/2017JA024156.

Denton MH, Ulich T, Turunen E. 2009. Modification of midlatitude ionospheric parameters in the F2 layer by persistent high-speed solar wind streams. Space Weather 7: S04006. https://doi.org/ 10.1029/2008SW000443.

Eastwood JP, Nakamura R, Turc L, Mejnertsen L, Hesse M. 2017. The scientific foundations of forecasting magnetospheric space weather. Space Sci Rev 212: 1221-1252. https://doi.org/10.1007/ s11214-017-0399-8

Emslie AG, Vlahos L. 1980. Radiation signatures from a locally energized flaring loop. Astrophys J 242: 359-373. https://doi.org/ $10.1086 / 158469$.

Emslie AG, Brown JC. 1980. The polarization and directivity of solar-flare hard X-ray bremsstrahlung from a thermal source. Astrophys J 237: 1015-1023. https://doi.org/10.1086/157947.

Escoubet CP, Fehringer M, Goldstein M. 2001. Introduction: The Cluster mission. Ann Geophys 19: 1197-1200. https://doi.org/ 10.5194/angeo-19-1197-2001.

Escoubet CP, Taylor MGGT, Masson A, Laakso H, Volpp J, et al. 2013. Dynamical processes in space: Cluster results. Ann Geophys 31: 1045-1059. https://doi.org/10.5194/angeo-31-1045-2013.

Escoubet CP, Masson A, Laakso H, Goldstein ML. 2015. Recent highlights from Cluster, the first 3-D magnetospheric mission. Ann Geophys 33: 1221-1235.

Fabiani S, Muleri F. 2014. Astronomical X-ray polarimetry. Astronomia e Astrofisica, Aracne editrice.

Fludra A, Doyle JG, Metcalf T, Lemen JR, Phillips KJH, et al. 1995. Evolution of two small solar flares. A\&A 303: 914.

Forte R, Jefferies SM, Berrilli F, Del Moro D, Fleck B, et al. 2018. The MOTH II Doppler-Magnetographs and Data Calibration Pipeline. Space Weather Heliosph: Processes Forecasts Proc Int Astron Union: IAU Symp 335: 335-339. https://doi.org/10.1017/ S1743921318000029.

Futaana Y, Barabash S, Grigoriev A, Holmström M, Kallio E, et al. 2006. First ENA observations at Mars: subsolar ENA jet. Icarus 182: 413-423. https://doi.org/10.1016/j.icarus.2005.08.024.

Galli A, Wurza P, Bochslera P, Barabashb S, Grigoriev A, et al. 2008. First observation of energetic neutral atoms in the Venus environment. Planet Space Sci 56: 807-811. https://doi.org/ 10.1016/j.pss.2007.12.011.

Garnier P, Dandouras I, Toublanc D, Brandt PC, Roelof EC, Mitchell DG, Krimigis SM, Krupp N, Hamilton DC, Waite H. 2007. The exosphere of Titan and its interaction with the kronian magnetosphere: MIMI observations and modeling. Planet Space Sci 55: 165-173.

Goldstein J, McComas DJ. 2013. Five years of stereo magnetospheric imaging by TWINS. Space Sci Rev 180: 39-70. https://doi. org/10.1007/s11214-013-0012-8.

Greenwald RA, Baker KB, Dudeney JR, Pinnock M, Jones TB, et al. 1995. DARN/SuperDARN: a global view of high-latitude convection. Space Sci Rev 71: 763-796. https://doi.org/10.1007/ BF00751350.

Guo J, Emslie AG, Kontar EP, Benvenuto F, Massone AM, et al. 2012a. Determination of the acceleration region size in a loopstructured solar flare. $A \& A$ 543: A53. https://doi.org/10.1051/ 0004-6361/201219341.

Guo J, Emslie AG, Massone AM, Piana M. 2012b. Properties of the acceleration regions in several loop-structured solar flares. ApJ 755: 32. https://doi.org/10.1088/0004-637X/755/1/32.

Haberreiter M, Delouille V, Del Zanna G, Dammasch I, Dominique M, et al. 2015. Modeling the EUV/UV irradiance within the FP7 SOLID project. Geophys Res Abstr 17: EGU2015-14484. http:// meetingorganizer.copernicus.org/EGU2015/EGU2015-14484.pdf.

Hardi P, Abbo L, Andretta V, Auchère F, Bemporad A, et al. 2012. Solar magnetism eXplorer (SolmeX). Exploring the magnetic field in the upper atmosphere of our closest star. Exp Astron 33: 271303. https://doi.org/10.1007/s10686-011-9271-0.

Heilig B, Lühr H. 2013. New plasma pause model derived from CHAMP field-aligned current signatures. Ann Geophys 31: 529-539. https://doi.org/10.5194/angeo-31-529-2013. 
Ippolito A, Pommois P, Zimbardo G, Veltri P. 2005. Magnetic connection from the Earth to the solar corona, flare positions and solar energetic particle observations. $A \& A$ 438: 2 . https://doi.org/ 10.1051/0004-6361:20052776.

Keil W. 2007. Radiation effects on spacecraft and countermeasurements, selected cases. In: Space weather - research towards applications in Europe. Lilensten J (Ed.), Astrophysics and Space Science library 344, Springer, Dordrecht, The Netherlands.

Kerr GS, Fletcher L, Russell AJB, Allred JC. 2016. Simulations of the Mg II k and Ca II 8542 lines from an Alfvén Wave-heated flare chromosphere. Astrophys J 827: 2. https://doi.org/10.3847/0004637X/827/2/101.

Koskinen HEJ, Baker DN, Balogh A, Gombosi T, Veronig A, Von Steiger R. 2017. Achievements and challenges in the science of space weather. Space Sci Rev 212(3-4): 1137-1157. https://doi. org/10.1007/s11214-017-0390-4.

Krimigis SM, Mitchell DG, Hamilton DC, Krupp N, Livi S, et al. 2005. Dynamics of Saturn's magnetosphere from MIMI during Cassini's orbital insertion. Science 307: 1270-1273. https://doi. org/10.1126/science.1105978.

Krucker S, Battaglia M, Cargill PJ, Fletcher L, Hudson HS, et al. 2008. Hard X-ray emission from the solar corona. Astron Astrophys Rev 16: 155. https://doi.org/10.1007/s00159-008-0014-9.

La Tessa C, Di Fino L, Larosa M, Narici L, Picozza P, et al. 2009. Estimate of the space station thickness at a USLab site using ALTEA measurements and fragmentation cross sections. Nuclear Inst Methods B 267: 3383-3387. https://doi.org/10.1016/j.nimb. 2009.06.107.

Lapenta G, Pierrard V, Keppens R, Markidis S, Poedts S, et al. 2013. SWIFF: space weather integrated forecasting framework. J Space Weather Space Clim 3: A05. https://doi.org/10.1051/swsc/2013027.

Larosa M, Agostini F, Casolino M, De Santis C, Di Fino L, et al. 2011. Ion rates in the International Space Station during the December 2006 Solar Particle Event. J Phys G: Nucl Part Phys 38: 095102. https://doi.org/10.1088/0954-3899/38/9/095102.

Laurenza M, Alberti T, Marcucci MF, Consolini G, Jacquey C, et al. 2019. Estimation of the particle radiation environment at 11 point and in the near-earth space. Astrophys $J$ 873: 112. https://doi.org/ 10.3847/1538-4357/ab0410.

Laurenza M, Vecchio A, Storini M, Carbone V. 2014. Drift effects on the galactic cosmic ray modulation. Astrophys $J$ 781: 71 . https://doi.org/10.1088/0004-637X/781/2/71.

Laurenza M, Vecchio A, Storini M, Carbone V. 2012. Quasi-biennial modulation of galactic cosmic rays. Astrophys $J$ 749: 167. https://doi.org/10.1088/0004-637X/749/2/167.

Lester M. 2013. The Super Dual Auroral Radar Network (SuperDARN): an overview of its development and science. $A d v$ Polar Sci 24(1): 1-11. https://doi.org/10.3724/SP.J.1085.2013. 00001.

Lilensten J, Belehaki A. 2009. Developing the scientific basis for monitoring, modelling and predicting space weather. Acta Geophys 57: 1-14. https://doi.org/10.2478/s11600-008-0081-3.

Lilensten J, Coates AJ, Dehant V, Dudok de Wit T, Horne RB, et al. 2014. What characterizes planetary space weather? Astron Astrophys Rev 22: 79https://doi.org/10.1007/s00159-014-0079-6.

Lin RP, Hudson HS. 1976. Non-thermal processes in large solar flares. Sol Phys 50: 153. https://doi.org/10.1007/BF00206199.

Lingri D, Mavromichalaki H, Belov A, Eroshenko E, Yanke V, et al. 2016. Solar activity parameters and associated forbush decreases during the minimum between cycles 23 and 24 and the ascending phase of cycle 24. Solar Phys 291(3): 1025-1041. https://doi.org/ 10.1007/s11207-016-0863-8.
Lockwood M, Fazakerley A, Opgenoorth H, Moen J, van Eyken AP, et al. 2001. Coordinated Cluster and ground-based instrument observations of transient changes in the magnetopause boundary layer during an interval of predominantly northward IMF: relation to reconnection pulses and FTE signatures. Ann Geophys 19: 1613-1640. https://doi.org/10.5194/angeo-19-1613-2001.

Massone AM, Piana M. 2013. The use of electron maps to constrain some physical properties of solar flares. Solar Phys 283: 177-186. https://doi.org/10.1007/s11207-011-9844-0.

Magrì M, Oliviero M, Severino G. 2008. Accurate intensity velocity phase difference in the potassium resonance line obtained with VAMOS. Solar Phys 247: 15. https://doi.org/10.1007/ s11207-007-9035-1.

Mangano V, Massetti S, Milillo A, Plainaki C, Orsini S, Leblanc F. 2015. THEMIS Na exosphere observations of Mercury and their correlation with in-situ magnetic field measurements by MESSENGER. Planet Space Sci 115: 102-109. https://doi.org/10.1016/j. pss.2015.04.001.

Mann G, Breitling F, Vocks C, et al. 2018. Tracking of an electron beam through the solar corona with LOFAR. A\&A 611: A57. https://doi.org/10.1051/0004-6361/201629017.

Mannucci AJ, Dickson J, Duncan C, Hurst K. 2010. GNSS Geospace Constellation (GGC): a CubeSat space weather mission concept. Jet Propulsion Lab., California Inst. of Technology, TR, Pasadena, CA. http://www8.nationalacademies.org/SSBSurvey/DetailFileDisplay. aspx?id=881

Marcucci MF, Coco I, Ambrosino D, Amata E, Milan SE, et al. 2008. Extended SuperDARN and IMAGE observations for northward IMF: evidence for dual lobe reconnection. J Geophys Res 113: A02204. https://doi.org/10.1029/2007JA012466.

Martucci M, Munini R, Boezio M, Di Felice V, Adriani O, et al. 2018. Proton fluxes measured by the PAMELA experiment from the minimum to the maximum solar activity for solar Cycle 24. Astrophys J 854: L2. https://doi.org/10.3847/2041-8213/aaa9b2.

Mavromichalaki H, Papaioannoua A, Plainakia C, Sarlanisa C, Souvatzoglou G, et al. 2011. Applications and usage of the realtime Neutron Monitor Database. Adv Space Res 47(12): 2210 2222. https://doi.org/10.1016/j.asr.2010.02.019.

Mauk BH, Mitchell DG, Krimigis SM, Roelof EC, Paranicas CP. 2003. Energetic neutral atoms from a trans-Europa gas torus at Jupiter. Nature. 421: 920-922. https://doi.org/10.1038/nature01431.

McComas DJ, Allegrini F, Baldonado J, Blake B, Brandt PC, et al. 2009. The two wide-angle imaging neutral-atom spectrometers (TWINS) NASA mission-of-opportunity. Space Sci Rev 142: 157. https://doi.org/10.1007/s11214-008-9467-4.

Messerotti M. 2018. Solar radio spectrography: comprehensive diagnostics for space weather applications. In: Proc. 2018 2nd URSI Atlantic Radio Science Meeting (AT-RASC), IEEE Explore Digital Library. https://doi.org/10.23919/URSI-AT-RASC.2018.8471360.

Messerotti M. 2019. Radio science for space weather. In: Invited, Proc. 2019 URSI Asia-Pacific Radio Science Conference (APRASC), IEEE Xplore Digital Library. In press.

Mewaldt RA, Leske RA, Stone EC, Barghouty AF, Labrador AW, et al. 2009. Stereo observations of energetic neutral hydrogen atoms during the 2006 December 5 solar flare. Astrophys J Lett 693: 1 . https://doi.org/10.1088/0004-637X/693/1/L11.

Miroshnichenko LI. 2018. Retrospective analysis of GLEs and estimates of radiation risks. J Space Weather Space Clim 8: A52. https://doi.org/10.1051/swsc/2018042.

Mitchell DG, Brandt PC, Roelof EC, Hamilton DC, Retterer KC, et al. 2003. Global imaging of $\mathrm{O}^{+}$from IMAGE/HENA. Space Sci Rev 109: 63. https://doi.org/10.1023/B:SPAC.0000007513.55076.00. 
Milillo A, Fujimoto M, Kallio E, Kameda S, Leblanc F, et al. 2010. The BepiColombo mission: an outstanding tool for investigating the Hermean environment. Planet Space Sci 58(1-2): 40-60. https://doi.org/10.1016/j.pss.2008.06.005.

Mura A, Wurz P, Lichtenegger HIM, Schleicher H, Lammer H, et al. 2009. The sodium exosphere of Mercury: comparison between observations during Mercury's transit and model results. Icarus 200(1): 1-11. https://doi.org/10.1016/j.icarus.2008. 11.014.

Mura A, Adriani A, Altieri F, Connerney JEP, Bolton SJ, et al. 2017. Infrared observations of Jovian aurora from Juno's first orbits: main oval and satellite footprints. Geophys Res Lett 44(11): 5308-5316. https://doi.org/10.1016/j.icarus.2008.11.01410.1002/2017GL072954.

Narici L, Casolino M, Di Fino L, Larosa M, Larsson O, et al. 2012. Iron flux inside the International Space Station is measured to be lower than predicted. Rad Meas 47: 1030-1034. https://doi.org/ 10.1016/j.radmeas.2012.07.006.

Narici L, Casolino M, Di Fino L, Larosa M, Picozza P, et al. 2015. Radiation survey in the International Space Station. J Space Weather Space Clim 5: A37. https://doi.org/10.1051/swsc/2015037.

Ngwira C, Pulkkinen A, Bernabeu E, Eichner J, Viljanen A, Crowley G. 2015. Characteristics of extreme geoelectric fields and their possible causes: localized peak enhancements. Geophys Res Lett 42: 6916-6921. https://doi.org/10.1002/2015GL065061.

Opgenoorth HJ, Lockwood M, Alcaydé D, Donovan E, Engebretson MJ. 2001. Coordinated ground-based, low altitude satellite and Cluster observations on global and local scales during a transient post-noon sector excursion of the magnetospheric cusp. Ann Geophys 19: 1367-1398.

Orsini S, Livi S, Torkar K, Barabash S, Milillo A, et al. 2010. SERENA: a suite of four instruments (ELENA, STROFIO, PICAM and MIPA) on board BepiColombo-MPO for particle detection in the Hermean Environment. BepiColombo Special Issue: Planet Space Sci 58: 166-181. https://doi.org/10.1016/j.pss.2008.09.012.

Orsini S, Mangano V, Milillo A, Plainaki C, Mura A, et al. 2018. Mercury Na exospheric emission as a proxy for CME transit. Sci Rep 8: 928. https://doi.org/10.1038/s41598-018-19163-x.

Paschmann G, Schwartz SJ, Escoubet CP, Haaland SE. 2005. Outer magnetospheric boundaries: Cluster results, Springer, Dordrecht, The Netherlands118/1-4, ISBN: 1-4020-3488-1.

Pellizzoni A, Buffa F, Egron E, Iacolina MN, Loru S, et al. 2018. High-resolution imaging of the solar chromosphere in the centimetre-millimetre band through single-dish observations. Proc. 2018 2nd URSI Atlantic Radio Science Conference (AT-RASC), IEEE Explore Digital Library. https://doi.org/10.23919/URSI-ATRASC.2018.8471502.

Peres G, Reale F, Serio S, Pallavicini R. 1987. Hydrodynamic flare modeling - Comparison of numerical calculations with SMM observations of the 1980 November 12 17:00 UT flare. Astrophys. J. 312: 895-908. https://doi.org/10.1086/164936.

Plainaki C, Negri B, Castronuovo M, Antonelli A. 2018. Towards an Italian Space Weather Infrastructure: The ASPIS project. Geophys Res Abstr 20: EGU2018-5239. https://meetingorganizer.copernicus. org/EGU2018/EGU2018-5239.pdf.

Plainaki C, Negri B, Castronuovo M. 2017. Proposal for a national Space Weather Infrastructure. SAIT 2017, Padova (Italy).

Plainaki C, Lilensten J, Radioti A, Andriopoulou M, Milillo A, et al. 2016. Planetary space weather: Scientific aspects and future perspectives. J Space Weather Space Clim 6: A31. https://doi.org/ $10.1051 /$ swsc/2016024.

Plainaki C, Mavromichalaki H, Laurenza M, Gerontidou M, Kanellakopoulos A, Storini M. 2014. The Ground Level Enhancement of 2012 May 17: Derivation of solar proton event properties through the application of the NMBANGLE PPOLA model. Astrophys J 785: 160. https://doi.org/10.1088/0004-637X/785/2/160.

Plainaki C, Mavromichalaki H, Belov A, Eroshenko E, Andriopoulou M, Yanke V. 2010. A new version of the neutron monitor based anisotropic GLE model: Application to GLE60. Sol Phys 264(1): 239-254. https://doi.org/10.1007/ s11207-010-9576-6.

Plainaki C, Belov A, Eroshenko E, Mavromichalaki H, Yanke V. 2007. Modeling ground level enhancements: Event of 20 January 2005. J Geophys Res [Space Phys] 112: 4102. https://doi.org/ 10.1029/2006JA011926.

Plainaki C, Mavromichalaki H, Belov A, Eroshenko E, Yanke V. 2009a. Modeling the solar cosmic ray event of 13 December 2006 using ground level neutron monitor data. Adv Space Res 43: 474-479. https://doi.org/10.1016/j.asr.2008.07.011.

Plainaki C, Mavromichalaki H, Belov A, Eroshenko E, Yanke V. 2009b. Neutron monitor asymptotic directions of viewing during the event of 13 December 2006. Adv Space Res 43: 518-522. https://doi.org/10.1016/j.asr.2008.09.007.

Plainaki C, Belov A, Eroshenko E, Kurt V, Mavromichalaki H, Yanke V. 2005. Unexpected burst of solar activity recorded by neutron monitors during October November 2003. Adv Space Res 35: 691-696. https://doi.org/10.1016/j.asr.2004.11.023.

Pollock CJ, Asamura K, Balkey MM, Burch JL, Funsten HO, et al. 2001. First medium energy neutral atom (MENA) Images of Earth's magnetosphere during substorm and storm-time. Geophys Res Lett 28: 1147-1150. https://doi.org/10.1029/2000GL012641.

Procházka O, Milligan RO, Allred JC, Kowalski AF, Kotrč P, et al. 2017. Suppression of Hydrogen Emission in an X-class Whitelight Solar Flare. Astrophys J 837: 46. https://doi.org/10.3847/ 1538-4357/aa5da8.

Pulkkinen A, Bernabeu E, Thomson A, Viljanen A, et al. 2017. Geomagnetically induced currents: Science, engineering, and applications readiness. Space Weather 15: 828-856. https://doi. org/10.1002/2016SW001501.

Pulkkinen A, Bernabeu E, Eichner J, Viljanen A, Ngwira C. 2015. Regional-scale high-latitude extreme geoelectric fields pertaining to geomagnetically induced currents. Earth Planets Space 67: 93. https://doi.org/10.1186/s40623-015-0255-6.

Radicella SM. 2007. Ionosphere/positioning and telecommunications. In: Space Weather Research towards Applications in Europe, Lilensten J, (Ed.), Astrophysics and Space Science library 344, Springer. pp. 125-127.

Reid HAS, Kontar EP. 2018. Solar type III radio burst time characteristics at LOFAR frequencies and the implications for electron beam transport. $A \& A$ 614: A69. https://doi.org/10.1051/ 0004-6361/201732298.

Renotte E, Alia A, Bemporad A, Bernier J, Barmanti Cet al. 2015. Design status of ASPIICS, an externally occulted coronagraph for PROBA-3. Solar Physics and Space Weather Instrumentation VI Proceedings 96040A. https://doi.org/10.1117/12.2186962.

Rice DD, Sojka JJ, Eccles JV, Raitt JW, Brady JJ, Hunsucker RD. 2011. First results of mapping sporadic E with a passive observing network. Space Weather 9: S12001. https://doi.org/10.1029/ 2011SW000678.

Rizzo A, Narici L, Messi R, Cipollone P, De Donato C, et al. 2018. A compact Time-Of-Flight detector for space applications: The LIDAL system. Nucl Instrum Methods Phys Res Sect A 898: 98104. https://doi.org/10.1016/j.nima.2018.05.009.

Rodriguez F, Ronchini R, Di Rollo S, De Franceschi G, Cesaroni C, et al. 2018. The ionosphere prediction service project. Geophys Res Abstr 20: EGU2018-15908. https://meetingorganizer.copernicus.org/EGU2018/EGU2018-15908.pdf. 
Romano P, Guglielmino SL, Cristaldi A, Ermolli I, Falco M, et al. 2014. Evolution of the magnetic field inclination in a forming penumbra. Astrophys J 784: 10. https://doi.org/10.1088/0004637X/784/1/10.

Romano V, Pau S, Pezzopane M, Spogli L, Zuccheretti L, et al. 2013. eSWua: A tool to manage and access GNSS ionospheric data from mid-to-high latitudes. Ann Geophys 56(2): R0223. https://doi.org/10.4401/ag-6244.

Romoli M, Landini F, Antonucci E, Andretta V, Berlicki A, et al. 2017. METIS: The visible and UV coronagraph for solar orbiter. Proc SPIE 10563: 105631M. https://doi.org/10.1117/12.2304274.

Schrijver CJ, Kauristie K, Aylward AD, Denardini CM, Gibson SE, et al. 2015. Understanding space weather to shield society: A global road map for 2015-2025 commissioned by COSPAR and ILWS. Adv Space Res 55: 2745-2807. https://doi.org/10.1016/ j.asr.2015.03.023.

Schwartz SJ, Horbury T, Owen C, Baumjohann W, Nakamura R, et al. 2009. Cross-scale: multi-scale coupling in space plasmas. Exp Astron 23: 1001-1015. https://doi.org/10.1007/s10686-008-9085-x.

Shinbori A, Otsuka Y, Tsugawa T, Nishioka M, Kumamoto A, et al. 2018. Temporal and spatial variations of storm time midlatitude ionospheric trough based on global GNSS-TEC and Arase satellite observations. Geophys Res Lett 45: 7362-7370. https://doi.org/ 10.1029/2018GL078723.

Søraas F, Sørbø M. 2013. Low altitude observations of ENA from the ring current and from the proton oval. J Atmos Sol Terr Phys 99: 104-110. https://doi.org/10.1016/j.jastp.2012.10.003.

Storini M, Signoretti F, Re P, Diego M, et al. 2015. Cosmic ray intensity for about five solar cycles. J Phys Conf Ser 632(1): 012065.

Susino R, Bemporad A. 2016. Determination of coronal mass ejection physical parameters from a combination of polarized visible light and UV Ly $\alpha$ observations. Astrophys $J$ 830: 58. https://doi.org/10.3847/0004-637X/830/2/58.

Susino R, Bemporad A, Dolei S, Vourlidas A. 2013. Study of a coronal mass ejection with SOHO/UVCS and STEREO data. Adv Space Res 52(5): 957-962. https://doi.org/10.1016/j.asr.2013.05.017.

Tomassetti N, Orcinha M, Barão F, Bertucci B. 2017. Evidence for a time lag in solar modulation of galactic cosmic rays. Astrophys $J$ Lett 849: L32. https://doi.org/10.3847/2041-8213/aa9373.

Tomassetti N, Barão F, Bertucci B, Fiandrini E, Figueiredo JL, et al. 2018. Testing diffusion of cosmic rays in the heliosphere with proton and helium data from AMS. Phys Rev Lett : 121, 251104. https://doi.org/10.1103/PhysRevLett.121.251104.

Tozzi R, Coco I, De Michelis P, Giannattasio F. 2019a. Latitudinal dependence of geomagnetically induced currents during geomagnetic storms. Annals of Geophysics 62: GM448. https://doi.org/ 10.4401/ag-7788.

Tozzi R, De Michelis P, Coco I, Giannattasio F. 2019b. A preliminary risk assessment due to geomagnetically induced currents over the Italian territory. Space Weather 17: 46-58. https://doi.org/10.1029/2018SW002065.

Trattner KJ, Fuselier SA, Petrinec SM, Yeoman TK, Mouikis C, Kucharek H, Reme H. 2005. Reconnection sites of spatial cusp structures. J Geophys Res 110: A04207. https://doi.org/10.1029/ 2004JA010722.

Treumann RA. 2009. Fundamentals of collisionless shocks for astrophysical application, 1. Non-relativistic shocks. Astron Astrophys Rev 17: 409-535. https://doi.org/10.1007/s00159-009-0024-2.

Usoskin IG, Gil A, Kovaltsov GA, Mishev AL, Mikhailov VV. 2017. Heliospheric modulation of cosmic rays during the neutron monitor era: calibration using PAMELA data for 2006-2010. J Geophys Res Space Phys 122: 3875-3887. https://doi.org/ 10.1002/2016JA023819.
Vaivads A, Retinò A, Soucek J, Khotyaintsev YV, Valentini F, et al. 2016. Turbulence Heating ObserveR - satellite mission proposal. J Plasma Phys 82(5): 905820501. https://doi.org/ $10.1017 /$ S0022377816000775.

Valek P, Goldstein J, McComas DJ, Ilie R, Buzulukova N, et al. 2013. Oxygen-hydrogen differentiated observations from TWINS: the 22 July 2009 storm. J Geophys Res118: 3377-3393. https://doi.org/10.1029/2012JA018465.

Vellante M, Piersanti M, Pietropaolo E. 2014. Comparison of equatorial plasma mass densities deduced from field line resonances observed at ground for dipole and IGRF models. $J$ Geophys Res 119: 2623-2633. https://doi.org/10.1002/2013JA019568.

Viscio MA, Viola N, Corpino S, Stesina F, Fineschi S, Fumentic F, Circi C. 2014. Interplanetary CubeSats system for space weather evaluations and technology demonstration. Acta Astronaut 104(2): 516-525. https://doi.org/10.1016/j.actaastro. 2014.06.005.

Viticchié B, Sánchez Almeida J, Del Moro D, Berrilli F. 2011. Interpretation of HINODE SOT/SP asymmetric Stokes profiles observed in the quiet Sun network and internetwork. $A \& A$ 526: A60. https://doi.org/10.1051/0004-6361/201015391.

Wild JA, Milan SE, Cowley SWH, Dunlop MW, Owen CJ, et al. 2003. Coordinated interhemispheric SuperDARN radar observations of the ionospheric response to flux transfer events observed by the Cluster spacecraft at the high-latitude magnetopause. Ann Geophys 21: 1807-1826. https://doi.org/10.5194/angeo-21-1807-2003.

Wu X, Ambrosi G, Azzarello P, Bergmann B, Bertucci B. 2019. Penetrating particle ANalyzer (PAN). Adv Space Res 63: 2672-2682. https://doi.org/10.1016/j.asr.2019.01.012.

Zaconte V, Casolino M, De Santis C, Di Fino L, La Tessa C, et al. 2010. The radiation environment in the ISS-USLab measured by ALTEA: spectra and relative nuclear abundances in the polar, equatorial and SAA regions. Adv Space Res 46: 797-799. https://doi.org/10.1016/j.asr.2010.02.032.

Zanini A, Ciancio V, Laurenza M, et al. 2017. Environmental radiation dosimetry at Argentine Antarctic Marambio Base $\left(64^{\circ} 13^{\prime} \mathrm{S}, 56^{\circ} 43^{\prime} \mathrm{W}\right)$ : preliminary results. J Environ Radioact 175-176: 149-157. https://doi.org/10.1016/j.jenvrad.2017.04.011.

Zharkova VV, Kuznetsov AA, Siversky TV. 2010. Diagnostics of energetic electrons with anisotropic distributions in solar flares. A\&A 512: A8. https://doi.org/10.1051/0004-6361/200811486.

Zharkova VV, Brown JC, Syniavskii DV. 1995. Electron beam dynamics and hard X-ray bremsstrahlung polarization in a flaring loop with return current and converging magnetic field. A\&A 304: 284.

Zheng Y, Ganushkina NY, Jiggens P, Jun I, Meier M, Minow JI, et al. 2019. Space radiation and plasma effects on satellites and aviation: Quantities and metrics for tracking performance of space weather environment models. Space Weather 17: 1384-1403. https://doi.org/10.1029/2018SW002042.

\section{Appendix A}

\section{Technical information on recent and on-going projects of the Italian Space Weather community}

Series of topic annexes related to the topic areas listed in Section 2.1 (see also Table 1). Each annex addresses a specific topic area and includes technical information on the related recent and on-going projects of the Italian Space Weather community. For further details on the projects themselves, the reader is referred to the individual weblinks. 


\section{Topic Annex n. 1}

Note 1.1. The FP7 F-CHROMA project (2013-2017) resulted in a notable advancing of our understanding of the energy dissipation and radiation processes in the flaring solar atmosphere. Moreover, a catalogue and archive facility providing access to both ground- and space-based datasets for well-observed flare events and flare atmospheric models to aid in data interpretation, was developed. Such material is of significant importance when studying the sources of circumeterrestrial Space Weather. We also note that the project had an impact beyond solar physics since it aimed to investigate what models can tell about the flares observed in other solar-type stars. INAF and UNICAT were among the participants of the F-CHROMA project. Detailed information on the F-CHROMA project can be found in:

https://www.fchroma.org/ and https://cordis.europa.eu/project/ $\mathrm{rcn} / 188819 /$ factsheet/en.

Note 1.2. Within the FP7 HESPE project (2010-2013) the development of flare prediction models was facilitated, hence, the utility of the project applications was extremely significant in the context of Space Weather. UNIGE had a leading role within this project. Detailed information on the HESPE project can be found in:

http://www.hespe.eu/ and https://cordis.europa.eu/project/ rcn/97529/factsheet/it.

Note 1.3. Our understanding of the solar flare phenomena was significantly improved after the H2020 FLARECAST project (2015-2017). A variety of statistical and machine learning techniques, including standard methods such as linear discriminant analysis, clustering and regression analysis, neural networks, as well as innovative approaches, was employed within the project. UNIGE and CNR provided significant contributions to this important international effort. Detailed information on the FLARECAST project can be found in:

http://flarecast.eu/ and https://cordis.europa.eu/project/rcn/ 193702/factsheet/en.

\section{Topic Annex n. 2}

Note 2.1. The FP7 "Solar system plasma Turbulence: Observations, inteRmittency and Multifractals" (STORM) project (2013-2015) was devoted to an in depth analysis of Solar System plasma turbulence from in situ data gathered by different spacecraft. The variations of the features of turbulence and intermittency with the solar activity were investigated and, moreover, an estimation of the expected impact was performed. The investigation was based on in situ space plasma data bases collected by different ESA missions (Giotto, Ulysses, Rosetta, Cluster and Venus Express) and on other satellite data bases (NASA's Cassini, Mars Global Surveyor and THEMIS). The approach revealed new universal properties of intermittent and anisotropic turbulence and multifractals in solar system plasmas (solar wind, the planetary foreshock and magnetosheath, both for the quasi-parallel and quasi-perpendicular geometry, the terrestrial magnetospheric cusps, the low latitude boundary layers of magnetized planets) and how these properties vary within the solar cycle and with the distance from the Sun. The outputs of the related studies, therefore, were very relevant to Space Weather science. INAF participated in this important project. Detailed information on the STORM project can be found in:

http://www.storm-fp7.eu/ and https://cordis.europa.eu/project/ $\mathrm{rcn} / 106507 /$ factsheet/en.

Note 2.2. The FP7 "Solar and Heliospheric Collisionless Kinetics" (SHOCK) project (2012-2015) brought together leading European groups working in the area of kinetic modeling of space plasma to enhance and accelerate the effective scientific exploitation of existing space plasma data sets and to maximize the scientific return of space missions. The main scope of the project was to identify synergies between space plasma modeling and data analysis, taking into consideration the increasing awareness that kinetic processes at small length scales and short time scales are crucial for a proper understanding of the fundamental processes governing the dynamics of heliospheric plasmas. Such studies are of fundamental importance for understanding Space Weather in the Solar System. UNIFI participated in this project. Detailed information on the SHOCK project can be found in:

http://www.project-shock.eu/home/ and https://cordis.europa. eu/project/rcn/100921/factsheet/en.

\section{Topic Annex n. 3}

Note 3.1. Within the FP7 "PLASMON: A new, groundbased data-assimilative modeling of the Earth's plasmasphere a critical contribution to Radiation Belt modeling for Space Weather purposes" project (2011-2014), stations extending from Italy to Finland (known also as the European quasiMeridional Magnetometer Array - EMMA) was realized under the leadership of UNIVAQ. Ultra Low Frequency (ULF) measurements from EMMA were used to derive near real-time equatorial plasma mass densities in the inner magnetosphere (1.6 $<L<6.2$, where $L$ is the McIlwain parameter). An assimilative model of the plasmasphere, which uses both EMMAderived plasma mass densities and equatorial electron densities derived from a worldwide network of whistler recording stations, was also developed. Within the same project it was shown that this plasmasphere model can be used to identify the regions where different kinds of plasma waves more efficiently interact with high-energy charged particles in the radiation belts to generate relativistic electron precipitation. Detailed information on the project can be found in:

http://plasmon.elte.hu/home.htm and https://cordis.europa. $\mathrm{eu} / \mathrm{project} / \mathrm{rcn} / 97831 /$ factsheet/en.

\section{Topic Annex n. 4}

Note 4.1. The FP7 "Environment for Human Exploration and RObotic Experimentation in Space" (e-HEROES) project (2012-2015) was dedicated to the exploitation of the data gathered during several European and international space missions, aiming to provide best estimate and prediction of the threats that future exploration missions to planetary bodies may encounter. In particular, the project provided useful information considering the characterization of the space environment, to be used during planning and implementation phases of manned or robotic space missions. A vast international synergy in the fields of solar and space physics, accompanied by numerous dissemination activities, was achieved. INAF and UNICAT were 
among the participants of the e-HEROES project. Detailed information on the project can be found in:

http://soteria-space.eu/eheroes/html/ and https://cordis. europa.eu/project/rcn/101382/factsheet/en.

Note 4.2. Radiation dosimetry campaigns have been performed in the Antarctic region in the framework of the "Cosmic Rays in Antarctica" (CORA) project (2013-2015), have been possible through a collaboration between Argentine and Italian institutions (INFN, INAF-IAPS). In the CORA project, due to the very few dosimetric data available in literature at high southern latitudes, accurate measurements are performed by using a set of different active and passive detectors. Special attention is dedicated to measure the neutron ambient dose equivalent in different energy ranges, by using an active detector, the Atomtex Rem Counter, for neutron energy in the $0.025 \mathrm{eV}-14 \mathrm{MeV}$ range and a set of passive bubble dosimeters, sensitive to thermal neutrons and neutrons in the $100 \mathrm{keV}-20 \mathrm{MeV}$ energy range (Zanini et al., 2017). Some additional information can be found in:

https://agenda.infn.it/event/11779/contributions/10548/ attachments/7708/8609/2016-07-07_Gruppo_5_-_HALCORD. pdf.

\section{Topic Annex n. 5}

Note 5.1. The "event catalogue" obtained by the HELIO project (2009-2012) included lists of events occurring in different locations in the heliosphere while the "feature catalogue" included information on the evolution of solar and heliospheric features. An additional output of the project was the development of a propagation model that linked different sets of observations. INAF-OATs participated in this innovative project. Detailed information on the FP7 HELIO project can be found in: http://www.helio-vo.eu/.

Note 5.2. ESPAS is a data e-infrastructure facilitating discovery and access to observations and model predictions of the near-Earth space environment (Belehaki et al., 2016). ESPAS facilitates the exploitation of multi-instrument multipoint science data for analysis. Since the data reside at the node of the provider, their data integrity and their distribution policy are protected. INGV participated in this project. Detailed information on the project can be found in:

https://www.espas-fp7.eu/portal/.

Note 5.3. The NMDB project resulted in a major advance of the use of cosmic ray data within Space Weather applications. INAF and UNIRoma 3 participated in this project. Detailed information on the FP7 NMDB project (2008-2009) can be found in:

http://www.nmdb.eu/ and in https://cordis.europa.eu/project/ rcn/86430/factsheet/en.

Note 5.4. The aim of the SWERTO project (2015-2017), funded at regional level (Regione Lazio FILAS-RU-20141028 grant), was the development of a Space Weather database based on multi-instrument data from space-based (PAMELA, ALTEA; see Sect. 2.3.2) and ground-based (IBIS, MOTHII; see Sect. 2.2) instruments (Berrilli et al., 2018), which are relevant to the determination of Space Weather conditions. This project allowed registered users to access scientific data from instrumentation available to UNITOV researchers through national and international collaborations. Intuitive software for the selection and visualization of such data and results from prototype forecasting codes for flare probability and SEP fluxes is provided. The SWERTO database provides both particle flux measurements recorded in space and spectro-polarimetric measurements of the solar photosphere, in an open access environment. Detailed information on the SWERTO database can be found in:

http://swerto.roma2.infn.it/.

Note 5.5. In the context of the FP7 SOLID project (20122015) the role of the solar irradiance variations in the terrestrial climate was investigated. The main goal of SOLID was to bring added value to the European spectral irradiance observations by combining all existing measurements and at the same time, by filling the temporal and spectral gaps through modeling and reconstruction of the spectral irradiance variations. In this way, the scientific community would be enabled to take full advantage of the potential value of the existing irradiance data sets. SOLID reduced the uncertainties in the irradiance time series providing uniform data sets of modeled and observed solar irradiance data from the beginning of the space era to the present including proper error and uncertainty estimates. Such a record is highly relevant for disciplines such as space exploration, Space Weather and heliospheric science in general. $I N A F-O A R$ participated in this project. Detailed information on the SOLID project can be found in:

https://www.mps.mpg.de/solar-variability/solid and https:// cordis.europa.eu/project/rcn/106571/factsheet/en.

Note 5.6. The FP7 "Space weather integrated forecasting framework" (SWIFF) project (2011-2014), led by Belgium, aimed at the development of an integrated framework for the modeling of Space Weather and the study of methods and software to address the linkage (coupling) between different physical processes developing simultaneously or in cascade. Mathematical models best suited to accurately represent such processes together with computational algorithms within a common integrated software infrastructure have been developed. Within the SWIFF project INAF-OATo was involved in the observational validation of numerical results for magnetic reconnection based on the analysis of post-CME current sheet evolution. Results from this integrated mathematical approach have been described by Lapenta et al. (2013). Detailed information on the SWIFF project can be found in:

http://www.swiff.eu/\# and https://cordis.europa.eu/project/ rcn/97040/brief/en.

Note 5.7. On the European Solar Telescope

The large-aperture European Solar Telescope (EST) will be realized in the Canary Islands, one of the first-class sites for astronomical observations, and will be optimized for simultaneous multiple wavelength spectral and spectro-polarimetric observations. The EST project is supported by the European Association for Solar Telescopes (EAST), currently formed by solar physicists from 18 European countries (including Italy), which intends to develop, construct and operate the telescope. EST is in the European Strategy Forum on Research Infrastructures (ESFRI) Roadmap since 2016 and will enter the Implementation Phase in 2021. First light is planned for 2027.

- The FP7 "EST: The large aperture European Solar Telescope" project (2008-2011) involved 29 European partners, plus 9 collaborating institutions, from 15 different 
countries. The project was focused on the conceptual design study of EST and successfully demonstrated the scientific, technical and financial feasibility of EST. The conceptual design was possible thanks to the co-funding allocated specifically by the EU and the combined efforts of many scientists and engineers committed to developing new ideas to make this facility a unique infrastructure to study the Sun. The Italian institutions participating to the EST consortium were INAF, UNITOV and SRS Engineering. Detailed information on the project can be found in http://www.est-east.eu/ds/ and https://cordis. europa.eu/project/rcn/88350/factsheet/en.

- The FP7 "SOLARNET - High-resolution Solar Physics Network" (2013-2017) project brought together and integrated major European research infrastructures in the field of high resolution solar physics, in order to promote their coordinated use and development. SOLARNET (http:// solarnet-project.eu/home) was conceived as a framework to ensure access to state-of-the-art facilities and data archives, as well as a source for collaborations aiming at the development of tools and prototypes for innovative instruments and data processing of importance for the future operation of EST. Several Italian Institutions, namely $C N R$, INAF, UNICAL, UNITOV and private company SRS Engineering Design SRL participated in the SOLARNET project. Detailed information on the project can be found in http://www.est-east.eu/est/index.php/solarnet and https:// cordis.europa.eu/project/rcn/108645/factsheet/en.

- The H2020 "SOLARNET - Integrating High Resolution Solar Physics" (2019-2022) project aims at integrating the most important European infrastructures in the field of high-resolution solar physics. H2020-SOLARNET includes all pertinent European research institutions, infrastructures, and data repositories. The added contribution from non-European research institutions and private companies maximizes the impact on global scale. Several Italian Institutions, namely INAF, UNICAL, UNITOV and private companies ADS. International Srl and BDP Engineering \& Manufacturing ScarL participate in the H2020-SOLARNET project. Detailed information on the project can be found in http://www.est-east.eu/est/ index.php/solarnet-h2020 and https://cordis.europa.eu/ project/rcn/220943/factsheet/en.

- The H2020 "Getting Ready for the EST" (GREST) (2015-2018) and H2020-PRE-EST (2017-2021) projects are intended to take EST to the next level of development by undertaking crucial activities to improve the performance of the current state-of-the-art instrumentation. $C N R$, INAF, and UNITOV, and private company ADS International Srl participated in the H2020-GREST project whereas INAF, UNICAT, UNITOV participate in the H2020-PRE-EST project. Detailed information on the GREST project can be found in https://cordis.europa.eu/ project/rcn/194915/factsheet/en; https://cordis.europa.eu/ project/rcn/207484/factsheet/en; and http://www.est-east. eu/est/index.php/grest.

Note 5.8. Telespazio coordinated the Ionosphere Prediction Service (IPS) project (2015-2017) of the European Commission in the frame of the Galileo programme (Albanese et al., 2018). The aim of the IPS project was strongly related to Space
Weather: in particular, the project scope was to design and develop a prototype platform to translate the prediction and forecast of the ionosphere effects into a service customized for specific Global Navigation Satellite System (GNSS) user communities. UNITOV and INGV provided important contributions within the project. The IPS development comprised prototype service design activities as well as development and research activities. The latter ones constituted the scientific backbone of the project that provided models and algorithms for forecasting products as well. Detailed information on IPS can be found in http://ips.gsc-europa.eu.

Note 5.9. The PECASUS (Pan-European Consortium for Aviation Space Weather User Services) initiative aims for a global Space Weather information service centre as specified by the International Civil Aviation Organisation (ICAO). The related State Letter (AN 10/1-IND/17/11) was released in June 2017. The countries forming the PECASUS consortium are Finland (Lead), Belgium, UK, Poland, Germany, Netherlands, Italy, Austria, and Cyprus. At the moment Italy participates in the Consortium thanks to the contribution of INGV and ENAC. PECASUS will provide information on Space Weather that has the potential to affect communications, navigation and the health of passengers and crew. After a 2018 audition of the consortium by Space Weather and operational management experts nominated by the World Meteorological Organisation (WMO), PECASUS was declared fully compliant to all the addressed criteria (i.e. regarding institutional, operational, technical and communication/ dissemination aspects) with no areas for improvement identified. In the end of 2018, PECASUS was selected by ICAO as one of the three Global Space Weather information service centers to be prepared for real-time, 24/7 warnings on the expected impacts of Space Weather events on user systems. Detailed information on PECASUS can be found in http://pecasus.eu/.

Note 5.10. The upcoming Solar Activity MOF Monitor (SAMM; INAF-OAR) will provide solar magnetic field measurements. SAMM is a project for a double channel telescope with the goal of measuring the solar magnetic field. In 2014, a robotic tomographic telescope for the monitoring of the solar activity, based upon MOF technology, was proposed by Dal Sasso srl with its brand AVALON Instruments, in partnership with $I N A F-O A R$ (scientific advisor). The project was awarded with national funding in 2015 and it is a fruitful example of cooperation between research institutions and national industry.

Note 5.11. The Tor Vergata Solar Synoptic Telescope (TSST UNITOV) project started in 2011 in collaboration with the Institute for Astronomy-University of Hawaii. Financial support based on national funds has been recently provided (PRIN-MIUR 2017). The instrument consists of a double telescope for full disk solar dopplergrams and magnetograms using a MOF-based telescope operating in the Potasium K I 769.9 nm line, and a $\mathrm{H} \alpha$ Daystar SR-127 0.4A telescope.

\section{Appendix B}

Technical information on the ground-based Space Weather assets of the Italian scientific community

Note 1. The Interferometric BIdimensional Spectropolarimeter (IBIS), a high cadence, dual interferometer imaging 
spectro-polarimeter, performs imaging spectroscopy and polarimetry of the solar photosphere and chromosphere with high spatial and spectral resolution and large Field of View (FOV). IBIS has been built by the INAF-OAA with the support of UNIFI and UNITOV. It is currently operated by INAF in collaboration with the US National Solar Observatory at the Dunn Solar Telescope in New Mexico but a possible new arrangement in a European facility is under consideration.

Note 2. TSRS1.0-A stores high-resolution radio data and indices acquired until 2010. This archive represents a unique data set for post-event modeling contributing to Space Weather science.

Note 3. Solar observations of the solar photosphere and chromosphere are daily carried out at INAF-OACt by means of an equatorial spar equipped with a Cook refractor (150/ $2230 \mathrm{~mm}$ ), used to make drawings of sunspot groups and pores from visual observations and another 150-mm refractor (2300 $\mathrm{mm}$ focal length) feeding a Zeiss $\mathrm{H} \alpha$ Lyot filter (bandwidth of 0.025 or $0.050 \mathrm{~nm}$, tunable filter range of $\pm 0.1 \mathrm{~nm}$ ) and a Charge-Coupled Device (CCD) camera with a sensor of $3056 \times 3056$ pixels, a pixel size of 12 micron and a dynamical range of 16 bit. The program performed by means of these instruments includes: characterization of the sunspot groups visible daily on the solar disc, digital image acquisitions (every $10 \mathrm{~min})$ in the $\mathrm{H} \alpha$ line center $(656.28 \mathrm{~nm})$, monitoring of transient phenomena, like flares and active prominences, and digital image acquisition in the continuum at $656.78 \mathrm{~nm}$ (every hour).

Note 4. The PSPT (INAF-OAR) produces seeing-limited full-disk digital images in the blue continuum (409.4 nm, Full Width at Half Maximum (FWHM) $0.3 \mathrm{~nm}$ ), red continuum (607.1 nm, FWHM $0.5 \mathrm{~nm}$ ), CaII K (393.4 nm, FWHM $0.3 \mathrm{~nm}$ ), CaII K Narrow Band Wing (NBW) (393.6 nm, FWHM $0.1 \mathrm{~nm}$ ), and CaII K Narrow Band Core (NBC) (393.4 nm, FWHM $0.1 \mathrm{~nm}$ ), with an unprecedented $0.1 \%$ pixel-to-pixel relative photometric precision. The upcoming addition of two narrow band CaII $\mathrm{K}$ filters will also allow imaging of the CaII $\mathrm{K}$ core to wing ratio with nearly the same precision.

Note 5. The VAMOS (INAF-OACN) functionality is based on the use of a Magneto-Optical Filter (MOF). VAMOS studies the dynamics of the solar photosphere through the obtained dopplergrams, magnetograms, and photospheric images (K1 D1 line at $769.9 \mathrm{~nm}$ ). In particular, the photospheric intensity and velocity fluctuations are being measured and correlated. An $\mathrm{H} \alpha$ filter integrates the magnetograms obtained by VAMOS with high resolution chromosphere and solar perturbations observations.

Note 6. The Dome C East (DCE) ionospheric radar, in operation since 2013, and Dome C North (DCN) HF ionospheric radar, which started operation beginning of 2018, are located at the Concordia research station in the Antarctic region, nearby the south geomagnetic pole, and make part of the Super Dual Auroral Radar Network (SuperDARN) network that continuously observes the ionosphere from mid-latitudes to the polar regions in both the Southern and Northern hemispheres (http://vt.superdarn.org/tiki-index.php). Each of the two radars emits multi-pulse sequences from the geomagnetic pole toward the auroral latitudes and can register the backscattered signal by decameter electron density irregularities in the ionospheric $\mathrm{E}$ and $\mathrm{F}$ regions from 75 range gates, with $45 \mathrm{~km}$ gate length, along 16 directions with a $3.3^{\circ}$ separation for a total field of view area of $\sim 5 \times 10^{6} \mathrm{~km}^{2}$. From the analysis of the backscattered signals important parameters can be obtained, as back scatter Doppler shift, power and spectral width.

Note 7. Each geomagnetic observatory provides a robust and reliable continuous sampling of magnetic field with two identical systems at each site giving necessary redundancy and hence providing 24/7 data availability. The standard Italian observatory data product is an 1-min filtered version of the $1 \mathrm{~s}$ vector data. Filtering is according to the INTERMAGNET standard. INTERMAGNET is a consortium of observatories and operating institutes that agree and stipulate standards for worldwide magnetic observatories (http://www.intermagnet. org). The derived observatory data products include hourly, monthly and annual means of 1-min data.

Note 8. The SEGMA monitoring system is based on an automated procedure to detect field line resonance (FLR) frequencies from 1s geomagnetic field measurements recorded at the EMMA network (a meridional array of 27 magnetometer stations extending from Italy to Finland (http://geofizika.canet. hu/plasmon/) which also includes SEGMA). Inversion of FLR frequencies to obtain radial profiles of the equatorial plasma mass densities is made using a realistic time-dependent magnetospheric field model (evaluated from real-time solar wind and Dst data) and numerical computation of Magnetohydrodynamics (MHD) wave equations. Results are updated every $15 \mathrm{~min}$ and are publicly available at a dedicated server at UNIVAQ (http://plasmonserver.aquila.infn.it/EMMA_FLR_ DENSITY).

Note 9. The MOTHII, under good sky conditions at South Pole Solar Observatory (SPSO), can produce high quality solar full disk dopplergrams and magnetograms at two heights of the photospheric-chromospheric region of the Sun's atmosphere. The instrument operates in the $\mathrm{Na}(589 \mathrm{~nm})$ and $\mathrm{K}(770 \mathrm{~nm})$ lines and is based on magneto-optical filters for solar applications developed in the 1990s by a team from UNIRomal.

Note 10. The Antarctic region plateau of Dome C (coord: $75^{\circ} 06^{\prime} \mathrm{S} ; 123^{\circ} 20^{\prime} \mathrm{E}$ ) offers a unique opportunity for groundbased observations of the solar corona, due to the high altitude of the site (3233 $\mathrm{m}$ above sea level) and the large amount of the daily hours of observations. Based on its experience and involvement with the externally-occulted ASPIICS coronagraph for the ESA PROBA-3 formation-flying mission, INAF-OATo has developed the internally-occulted AntarctiCor coronagraph for ground-based observations of the polarized broad spectral band K-corona and of the narrow spectral band polarized emission of the coronal "Green-line" at $530.3 \mathrm{~nm}$. Other Italian institutes participating to this project are INAF-OAA, UNIFI, Observatory of Valle D'Aosta. 


\section{Appendix C}

\section{Acronym Definition}

\begin{tabular}{|c|c|}
\hline & Terminology \\
\hline $\mathrm{AC}$ & Anticoincidence System \\
\hline ACE & Advanced Composition Explorer \\
\hline ADS & Astrophysics Data System \\
\hline AGILE & Astro-Rivelatore Gamma a Immagini Leggero \\
\hline AIS & Advanced Ionospheric Sounder \\
\hline ALTCRISS & $\begin{array}{l}\text { Alteino Long Term Cosmic Ray Measurements on board the } \\
\text { International Space Station }\end{array}$ \\
\hline ALTEA & Anomalous Long Term Effects on Astronauts \\
\hline AMS & Alpha Magnetic Spectrometer \\
\hline $\begin{array}{l}\text { ASI SW } \\
\text { WG }\end{array}$ & ASI Space Weather Working Group \\
\hline ASPIS & ASI Space Weather Infrastucture \\
\hline CALET & Calorimetric Electron Telescope \\
\hline CCD & Charge-Coupled Device \\
\hline CIS & Cluster Ion Spectrometry \\
\hline CME & Coronal Mass Ejection \\
\hline CIR & Corotating Interaction Region \\
\hline COSPAR & Committee On Space Research \\
\hline CRIS & Cosmic Ray Isotope Spectrometer \\
\hline CSES & China Seismo-Electromagnetic Satellite \\
\hline DAMPE & DArk Matter Particle Explore \\
\hline DCE & Dome C East \\
\hline DCN & Dome C North \\
\hline DPU & Data Processing Unit \\
\hline EAS & Electron Analyser Systems \\
\hline EAST & European Association for Solar Telescopes \\
\hline $\mathrm{EC}$ & European Commission \\
\hline ECSS & European Cooperation for Space Standardization \\
\hline EFD & Electric Field Detector \\
\hline ELENA & Emitted Low-Energy Neutral Atoms \\
\hline EM & Engineering Model \\
\hline EMMA & European quasi-Meridional Magnetometer Array \\
\hline ENA & Energetic Neutral Atom \\
\hline ENAC & Ente Nazionale per l'Aviazione Civile \\
\hline EPHIN & Electron Proton and Helium Instrument \\
\hline ESC & Expert Service Center \\
\hline ESCAPE & Extreme Solar Coronagraphy Antarctic Program Experiment \\
\hline ESFRI & European Strategy Forum on Research Infrastructures \\
\hline EST & European Solar Telescope \\
\hline EUV & Extreme UltraViolet \\
\hline EUVST & Extreme UltraViolet Spectroscopic Telescope \\
\hline FAIR & Findable, Accessible, Interoperable, Reusable \\
\hline FAQ & Frequently Asked Questions \\
\hline FLR & Field Line Resonance (FLR) \\
\hline FM & Flight Model \\
\hline FOC & Full Operational Capability \\
\hline FOV & Field of view \\
\hline GCR & Galactic Cosmic Ray \\
\hline GEO & Geosynchronous Earth orbit \\
\hline GIC & Geomagnetically Induced Current \\
\hline GLE & Ground Level Enhancement \\
\hline GNSS & Global Navigation Satellite System \\
\hline GOES & Geostationary Operational Environmental Satellite \\
\hline GPD & Gas Pixel Detectors \\
\hline GPS & Global Positioning System \\
\hline GREST & Getting Ready for the EST \\
\hline GRID & Gamma Ray Imaging Detector \\
\hline HEPD & High Energy Particle Detector \\
\hline $\mathrm{HF}$ & High Frequency \\
\hline
\end{tabular}

(Continued)

Terminology

\begin{tabular}{|c|c|}
\hline HIS & Heavy Ion Sensor \\
\hline HSS & High Speed Streams \\
\hline IBIS & Interferometric BIdimensional Spectropolarimeter \\
\hline ICAO & International Civil Aviation Organisation \\
\hline ICME & Interplanetary Coronal Mass Ejection \\
\hline ILWS & International Living with A Star \\
\hline IMAGE & Imager for Magnetopause-to-Aurora Global Exploration \\
\hline INTERMAGNET & International Real-Time Magnetic Observatory Network \\
\hline IOC & Initial Operational Capability \\
\hline ISAS & Institute of Space and Astronautical Science \\
\hline ISS & International Space Station \\
\hline ISEE & Institute for Space-Earth Environmental Research \\
\hline ISWAT & International Space Weather Action Teams \\
\hline ISWI & International Space Weather Initiative \\
\hline IXPE & Imaging X-Ray Polarimetry Explorer \\
\hline JIRAM & Jovian Infrared Auroral Mapper \\
\hline LASCO & Large Angle and Spectrometric Coronagraph \\
\hline LEO & Lew Earth orbit \\
\hline LIDAL & Light Ion Detector for ALTEA \\
\hline LISA & Laser Interferometer Space Antenna \\
\hline LOFAR & Low-Frequency Array \\
\hline MCAO & Multiconjugate Adaptive Optics \\
\hline MCAL & Mini-Calorimeter \\
\hline MHD & Magnetohydrodynamics \\
\hline MIRACLE & $\begin{array}{l}\text { Magnetometers - Ionospheric Radars-Allsky Cameras Large } \\
\text { Experiment }\end{array}$ \\
\hline MMS & Magnetospheric Multiscale Mission \\
\hline MOF & Magneto-Optical Filter \\
\hline NIR & Near Infrared \\
\hline NMDB & Neutron Monitor Database \\
\hline NOAA & National Oeanic And Atmospheric Center \\
\hline NUV & Near Ultraviolet \\
\hline $\mathrm{O} 2 \mathrm{R}$ & Operations-to-Research \\
\hline PAMELA & $\begin{array}{l}\text { Payload for Antimatter Matter Exploration and Light-nuclei } \\
\text { Astrophysics }\end{array}$ \\
\hline PAN & Penetrating particle ANalyzer \\
\hline PAS & Proton Alpha Sensor \\
\hline PECASUS & $\begin{array}{l}\text { Pan-European Consortium for Aviation Space weather User } \\
\text { Services }\end{array}$ \\
\hline PI & Principal Investigator \\
\hline PSPT & Precision Solar Photometric Telescope \\
\hline QM & Qualification Model \\
\hline RFI & Radio Frequency Intereference \\
\hline RHESSI & Reuven Ramaty High Energy Solar Spectroscopic Imager \\
\hline SAMM & Solar Activity MOF Monitor \\
\hline SCORE & Sounding rocket COronagraphic Experiment \\
\hline SCR & Solar cosmic rays \\
\hline SDO & Solar Dynamics Observatory \\
\hline SEB & Single event burnout \\
\hline SEE & Single event effects \\
\hline SEGR & Single event gate rupture \\
\hline SEL & Single event latch-up \\
\hline SEP & Solar Energetic Particle \\
\hline SERENA & $\begin{array}{l}\text { Search for Exospheric Refilling and Emitted Natural Abundances } \\
\text { Experiment }\end{array}$ \\
\hline SME & Small and Medium-sized Enterprise \\
\hline SEGMA & South European Geomagnetic Array \\
\hline SEU & Single event upset \\
\hline SEL & Single Event Latch-up \\
\hline SEGR & Single Event Gate Rupture \\
\hline $\mathrm{SOHO}$ & Solar and heliospheric Observatory \\
\hline SPE & Solar particle event \\
\hline SPSO & South Pole Solar Observatory \\
\hline
\end{tabular}


(Continued)

\begin{tabular}{ll}
\hline & \multicolumn{1}{c}{ Terminology } \\
\hline SRT & Sardinia Radio Telescope \\
SSA & Space Situation Awareness \\
STEREO & Solar TErrestrial RElations Observatory \\
SuperDARn & Super Dual Auroral Network \\
SVIRCO & Studio Variazioni Intensità Raggi Cosmici \\
SWA & Solar Wind Analyser \\
SWERTO & Space WEeatherR TOr vergata university \\
SWICo & Space Weather Italian Community \\
SWxC & space weather information service center \\
TEC & Total Electron Content \\
TID & Total ionizing dose \\
TSRS & Trieste Solar Radio System \\
TSST & Tor Vergata Solar Synoptic Telescope \\
TWINS & Two Wide-angle Imaging Neutral-atom Spectrometers \\
UHF & Ultra high frequency \\
ULF & Ultra low frequency \\
UVCS & UV Coronagraph Spectrometer \\
VAMOS & Velocity and Magnetic Observations of the Sun \\
VHF & Very high frequency \\
VIS & Visible \\
VL & Visible light \\
WL & White Light \\
WMO & World Meteorological Organisation \\
\hline & \\
& \\
\hline &
\end{tabular}

\section{Institutions}

\begin{tabular}{ll} 
AMI & Aeronautica Militare Italiana \\
ASI & Agenzia Spaziale Italiana \\
CNR & Consiglio Nazionale delle Ricerche \\
ASTRON & Netherlands Institute for Radio Astronomy \\
CNSA & China National Space Administration \\
EOS & Unità di Esplorazione e Osservazione dell'Universo \\
ESA & European Space Agency \\
FBK & Fondazione Bruno Kessler \\
\hline
\end{tabular}

(continues)

\begin{tabular}{|c|c|}
\hline & Institutions \\
\hline IAPS & Istituto di Astrofisica e Planetologia Spaziali \\
\hline IFN & Istituto di Fotonica e Nanotecnologie \\
\hline INAF & Istituto Nazionale di Astrofisica \\
\hline INFN & Istituto Nazionale di Fisica Nucleare \\
\hline INGV & Istituto Nazionale di Geofisica e Vulcanologia \\
\hline IRA & Istituto di Radioastronomia \\
\hline ISAC & Istituto di Scienze dell'Atmosfera e del Clima \\
\hline JAXA & Japan Aerospace Exploration Agency \\
\hline JPL & Jet Propulsion Laboratory \\
\hline NASA & National Aeronautics and Space Administration \\
\hline OAA & Osservatorio Astrofisico di Arcetri \\
\hline OAC & Osservatorio Astronomico di Cagliari \\
\hline $\mathrm{OACN}$ & Osservatorio Astronomico di Capodimonte \\
\hline $\mathrm{OACt}$ & Osservatorio Astrofisico di Catania \\
\hline OAR & Osservatorio Astronomico di Roma \\
\hline OATo & Osservatorio Astrofisico di Torino \\
\hline OATs & Osservatorio Astronomico di Trieste \\
\hline SSDC & Space Science Data Center \\
\hline TNG & Telescopio Nazionale Galileo \\
\hline UNIBA & Università degli Studi di Bari \\
\hline UNICA & Università degli Studi di Cagliari \\
\hline UNICAL & Università della Calabria \\
\hline UNICAT & Università degli Studi di Catania \\
\hline UNIFI & Università degli Studi di Firenze \\
\hline UNIMI & Università degli Studi di Milano \\
\hline UNIPD & Università degli Studi di Padova \\
\hline UNIPG & Università degli Studi di Perugia \\
\hline UNIPV & Università degli Studi di Pavia \\
\hline UNIRoma1 & Sapienza Università di Roma \\
\hline UNIRoma3 & Università degli Studi Roma 3 \\
\hline UNITO & Università degli Studi di Torino \\
\hline UNITOV & Università degli Studi di Roma "Tor Vergata" \\
\hline UNITN & Università degli Studi di Trento \\
\hline UNITS & Università degli Studi di Trieste \\
\hline UNIVAQ & Università degli Studi dell'Aquila \\
\hline UNIVURB & Università degli Studi di Urbino \\
\hline URS & Unità di Ricera Scientifica \\
\hline
\end{tabular}

Cite this article as: Plainaki C, Antonucci M, Bemporad A, Berrilli F, Bertucci B, et al. 2020. Current state and perspectives of Space Weather science in Italy. J. Space Weather Space Clim. 10, 6. 